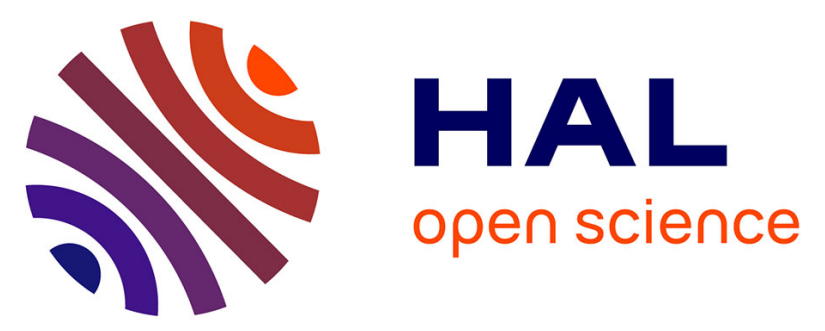

\title{
Preclinical Alzheimer's disease: a systematic review of the cohorts underlying the concept
}

Stéphane Epelbaum, Rémy Genthon, Enrica Cavedo, Marie Habert, Foudil

Lamari, Geoffroy Gagliardi, Simone Lista, Marc Teichmann, Hovagim

Bakardjian, Harald Hampel, et al.

\section{To cite this version:}

Stéphane Epelbaum, Rémy Genthon, Enrica Cavedo, Marie Habert, Foudil Lamari, et al.. Preclinical Alzheimer's disease: a systematic review of the cohorts underlying the concept. Alzheimer's \& Dementia: the Journal of the Alzheimer's Association, 2017, 13 (4), pp.454-467. 10.1016/j.jalz.2016.12.003 . hal-01672859

\section{HAL Id: hal-01672859 \\ https://hal.inria.fr/hal-01672859}

Submitted on 27 Dec 2017

HAL is a multi-disciplinary open access archive for the deposit and dissemination of scientific research documents, whether they are published or not. The documents may come from teaching and research institutions in France or abroad, or from public or private research centers.
L'archive ouverte pluridisciplinaire HAL, est destinée au dépôt et à la diffusion de documents scientifiques de niveau recherche, publiés ou non, émanant des établissements d'enseignement et de recherche français ou étrangers, des laboratoires publics ou privés. 


\section{Preclinical Alzheimer's disease: a systematic review of the cohorts underlying the concept}

Stéphane Epelbaum ${ }^{\mathrm{a}, \mathrm{b}}$, Rémy Genthon ${ }^{\mathrm{a}}$, Enrica Cavedo ${ }^{\mathrm{a}}$, Marie Odile Habert ${ }^{\mathrm{b}, \mathrm{c}}$, Foudil Lamari $^{\mathrm{d}}$, Geoffroy Gagliardi ${ }^{\mathrm{a}, \mathrm{b}}$, Simone Lista ${ }^{\mathrm{a}, \mathrm{e}, \mathrm{f}}$, Marc Teichmann ${ }^{\mathrm{a}, \mathrm{b}}$, Hovagim Bakardjian ${ }^{\mathrm{a}, \mathrm{e}, \mathrm{f}}$, Harald Hampel ${ }^{\mathrm{a}, \mathrm{b}, \mathrm{f}}$, Bruno Dubois ${ }^{\mathrm{a}, \mathrm{b}}$

a AP-HP, Groupe Hospitalier Pitié-Salpêtrière, Département de Neurologie, Institut de la mémoire et de la maladie d'Alzheimer, Groupe Hospitalier Pitié-Salpêtrière, $47 \mathrm{Bd}$ de l’Hôpital, 75013, Paris, France

b ICM - CNRS UMR 7225 - Inserm U 1127 - UPMC-P6 UMR S 1127, GH Pitié-Salpêtrière, 47 Bd de l'Hôpital, 75013, Paris, France

c AP-HP, Groupe Hospitalier Pitié-Salpêtrière, Département de médecine nucléaire, Groupe Hospitalier Pitié-Salpêtrière, 47 Bd de l'Hôpital, 75013, Paris, France

d AP-HP, Groupe Hospitalier Pitié-Salpêtrière, Laboratoire de Biochimie, Groupe Hospitalier Pitié-Salpêtrière, 47 Bd de l'Hôpital, 75013, Paris, France

e IHU-A-ICM - Paris Institute of Translational Neurosciences, Hôpital de la Pitié-Salpêtrière, 47 Bd de l'Hôpital, 75013, Paris, France

f AXA Research Fund \& UPMC Chair, Paris, France

\section{Abstract}

Preclinical Alzheimer's disease (AD) is a relatively recent concept describing an entity characterized by the presence of a pathophysiological biomarker signature characteristic for $\mathrm{AD}$ in the absence of specific clinical symptoms. There is rising interest in the scientific community to define such an early target population mainly due to failures of all recent clinical trials despite evidence of biological effects on brain amyloidosis for some compounds. A conceptual framework has recently been proposed for this preclinical phase of AD. However, few data exist on this silent stage of AD. We performed a systematic review in order to investigate how the concept is defined across studies. The review highlights the substantial heterogeneity concerning the three main determinants of preclinical AD: "normal cognition", "cognitive decline" and "AD pathophysiological signature". We emphasize the need for a harmonized nomenclature of the preclinical AD concept and standardized population-based and case-control studies using unified operationalized criteria. 


\section{Introduction}

The positivity of biomarkers of Alzheimer's disease (AD) before the occurrence of first clinical symptoms and dementia, supports the concept that $\mathrm{AD}$ is a continuum, and that it could be diagnosed before its clinical expression [1]. Intervention at such an early stage of the disease is considered to improve the chance of success because it would target potentially still reversible and less established and extensive pathological processes. The lack of clinical efficacy of trials using monoclonal antibodies targeting amyloid at a mild or moderate stage of the illness is further encouragement to shift the attention to the preclinical stage of the disease.

The concept of a preclinical stage of AD emerged mainly from clinico-pathological studies describing apparently cognitively normal individuals with the possibility of AD hallmark lesions in the brain.[2-5] The International Working Group-2 (IWG-2) and later the National Institute on Aging-Alzheimer's Association (NIA-AA) consortium each proposed a definition of the preclinical stage of $\mathrm{AD}[6,7]$. The recent release of consensual criteria should facilitate the harmonization and the quality of epidemiological and interventional research on preclinical AD [1].

Until now, little is known about the natural history of the preclinical state. Large epidemiological studies have been conducted or are still ongoing regarding the risk of dementia in the general population, but they are not strictly focusing on $\mathrm{AD}$, and even less on the identification of subjects with the preclinical form of the disease using AD biomarkers (For review see [8] ).

Per definition, people with preclinical AD lack the classical symptoms of the disease. However, the NIA-AA defines a stage of preclinical AD, with "subtle cognitive decline" [7]. This is due to the fact that most longitudinal epidemiological studies show the occurrence of decline, mainly in terms of psycho-motor speed and executive functions, years before the diagnosis of dementia [9, 10]. There is no consensual definition for "subtle cognitive changes" (i.e. "normal cognition" and "cognitive decline"). Likewise, an AD physiopathological biomarker profile was not required for study inclusion in these studies.

The present article, based on a systematic review of the literature on preclinical AD, aims at identifying the diagnostic approaches used by the leading groups in the field at this early stage of the disease. In particular three main issues concerning the concept of preclinical AD must be clarified: 1) the level of cognitive performance considered as "normal cognition" 2) the changes in cognitive performance considered as "cognitive decline", and 3) the best biomarkers or the best combination of them able to identify the "AD pathophysiological signature" in vivo. This review could support future clinical research in the field especially if a disease modifying drug demonstrates its efficacy.

\section{METHODS:}

\section{Search strategy and selection criteria}


The PubMed Database and ClinicalTrials.gov were searched for the terms "Preclinical Alzheimer's disease", "Preclinical Alzheimer disease", "Presymptomatic Alzheimer's disease", "Presymptomatic Alzheimer disease", "Asymptomatic Alzheimer's disease", "Asymptomatic Alzheimer disease", up to June 2016, without any language restriction. The terms had to be in the title or even in the abstract of the manuscript in order to include articles that would only refer to the concept of preclinical AD without studying it.

\section{Search Strategy Results and further classification of studies}

We identified 361 articles reporting "preclinical AD". They were categorized as "reviews" (for review, conceptual and perspective articles), "out of topic" (when despite the title or abstract of the article, no preclinical AD subject was included in the study), "neuropathological" (when AD diagnosis was pathologically established in subjects who died within one year of a cognitive evaluation considered as unimpaired), "genetic" when the study dealt with cognitively healthy carriers of causative mutations for familial AD, and "biomarker" when they comprised a biomarker based definition of the AD pathophysiology. They were further stratified in "cross sectional" or "longitudinal". Furthermore, we empirically chose to exclude articles with a sample size below 100 participants in order to focus on the major cohorts allowing for the study of the preclinical AD concept. The search strategy and distribution of the studies are reported in Fig 1. Fifty five studies from the "neuropathological", "genetic" and "biomarker" groups satisfied the above criteria and have been investigated. From each study, the total number of participants according to their diagnosis (healthy control, preclinical AD, NIA-AA preclinical AD stages and when appropriate mild cognitive impairment (MCI) and $\mathrm{AD}$ dementia participants) were extracted as well as their mean age, the percentage of $A P O E \& 4$ carriers and the cohort study from which they derived. As "Suspected non AD Pathophysiology" (SNAP) for biomarker based studies and "Primary age related taupathy" (PART) for neuropathological studies are two concepts that arose from the more systematic use of AD biomarkers and the rising interest in the earlier stages of AD [11], their number in studies on Preclinical AD were also considered. Finally, the way to define "normal cognition", "cognitive decline" and "AD pathophysiological signature" was analysed in each study. An overview of the studies' population and methodologies are provided in Tables 1 \& 2 respectively. The detailed description of the 55 studies and their methodology are provided in Supplementary Tables 1 and 2 .

\section{Cohorts allowing the study of preclinical AD}

Thirteen different cohorts of cognitively normal individuals for the investigation of preclinical $\mathrm{AD}$ were identified from these 55 publications. Nine of them are monocentric and currently recruiting as they were developed in the context of a clinical-research setting with an observational period ranging from 3 to 20 years. Each cohort characteristics were extracted from the published studies and from the cohorts' websites when available. Specifically, the latest published number of healthy elderly volunteers included in the cohort, the type of follow-up,(clinical routine or research), the mono or multicentric recruitment, the ethnicity and inclusion of minorities, the geographical origin of participants, the male/female ratios, the age of included participants, the selected criteria for normal cognition, the neuropsychological 
battery, the existence of cerebrospinal fluid biomarker or blood sampling, MRI, 18FDG-PET, Amyloid-PET and other biomarkers was reported (see Table $3 \&$ Supplementary Table 3). The number of studies in this review categorized by the cohort they emerge from are detailed in Supplementary Fig 1. The diverse cognitive tests are also presented in Fig 2 to clearly depict their frequency of use in the 13 cohorts.

\section{Clinical trials on preclinical AD}

In addition to the observational cohorts described above, the ClinicalTrials.gov website was employed for a detailed research on the drug trials available on the preclinical AD population. All trials mentioning "preclinical AD" as a target population with pathophysiological markers of $\mathrm{AD}$ as inclusion criteria in their study design were considered. Three trials were identified, 2 of which concerning familial AD as described in Table 4. This relatively low number of trials is due to the fact that most (8/11) trials listed on the "Clinicaltrial.gov" webpage (but excluded from this review) pertaining to the "preclinical Alzheimer's disease" search terms do not use pathophysiological markers at enrolment, thus being trials on the risk of developing MCI or dementia rather than on the more precise "preclinical AD" concept.

\section{RESULTS:}

\section{"Normal cognition"}

The concept of "normal cognition" is controversial. It is indeed hard to define whether a given individual can be considered as cognitively normal. Usually this is achieved by comparing his psychometric performance to that of a predefined age and educational level matched group on specific tests. In this case, there is no reference to his own cognitive abilities prior to the assessment. This individual factor, requiring longitudinal follow-up prior to inclusion, is almost never accounted for in studies on preclinical AD. Moreover, in the 55 studies selected, five $(9.1 \%)$ did not clearly specify what was considered as "normal cognition". Twenty-one studies (38.2\%) made use of the Clinical Dementia Rating scale (CDR) out of which thirteen (23.6\%) used exclusively the CDR score equal to 0 to classify participants as cognitively healthy. The remaining $29(52.7 \%)$ studies relied either on single Mini-mental State Examination (MMSE) or multiple cognitive tests, or on the clinical judgment of one investigators (see Table 2 for details). When cognitive tests were used, the clear definition of what was considered to be "pathological" was not always explicit. By contrast, in some cases it was described thoroughly [12]. MMSE scores when used as cut-off points between normality and impairment varied from $26[13,14]$ to 28 [15]. Interestingly, the MMSE cut-off scores, used in the studies, were higher than those necessary to be included in some of the cohorts (see Table 3 and below). Finally, the 3 clinical trials conducted on preclinical AD used different inclusion criteria (see Table 4).

Concerning the cohorts: The criterion used to define 'normal cognition' was heterogeneous as well. In seven out of the thirteen cohorts, the definition was based on the performance 
obtained on standard neuropsychological batteries. In the remaining cohorts, subjects were considered cognitively intact when they had a MMSE scores above 24 with a CDR score equal to 0 in the absence of depressive symptoms. The clinical and neuropsychological assessments were part of the study protocol for all considered cohorts, although the neuropsychological assessment used was not harmonized among cohorts (Fig. 3). The use of core biomarkers of $\mathrm{AD}$ was also heterogeneous. In most of the cohorts, the collection of biological and imaging markers was mainly restricted to a subsample of subjects. In addition to the physiopathological biomarkers, three studies collected EEG and three other reported post mortem neuropathological findings.

In terms of open source availability of data collected, not all of these studies are accessible to the scientific community. To our knowledge, the Alzheimer's Disease neuroimaging Initiative (ADNI), the Australian Imaging, the Biomarkers and Lifestyle Flagship Study of Ageing (AIBL), the Harvard Aging Brain Study (HABS), the Charles F. and Joanne Knight Alzheimer's Disease Research Centre (Knight ADRC) at Washington University School of Medicine, the National Alzheimer's Coordinating Centre (NACC) database, and the Wisconsin Registry for Alzheimer's Prevention (WRAP) are the only databases on preclinical $\mathrm{AD}$ patients allowing external investigators to access data throughout online available platforms and after appropriate review of projects submitted.

\section{"Cognitive decline/outcome"}

The definition of cognitive decline, as previously emphasized by the NIA-AA guidelines and in clinical trials in preclinical AD descriptions [7, 16-18], also raises some issues: if it is too strict (e.g. going from a CDR equal to 0 to a CDR equal to 1 ), the number of individuals with "preclinical AD" progressing to "clinical AD" will be very low and will require long-term studies (years if not decades) to draw conclusions on risk factors and progression of preclinical AD. Conversely, if the definition encompasses any slight change in cognition over time (e.g. an increase of a few seconds in a timed psycho-motor speed test), the risk of a low specificity and high number of false positive rises (i.e. temporary cognitive impairment unrelated to $\mathrm{AD}$ and disappearing during longer follow-up). In the reviewed studies, the strategy to define cognitive decline was heterogeneous (see table 2 for details). In the three clinical trials, different tests were used to evaluate cognitive decline (see Table 4). Contrarily to the other "determinants" of preclinical AD, the cognitive decline is not mandatory for diagnosis. Both hypothetical frameworks of preclinical AD recognize that the diagnosis can be made when there is 1) a normal cognition and 2) markers of AD pathophysiology [1, 7]. However, evidencing a cognitive decline (even when cognition remains normal with respect to normative data) in an individual is a strong supportive argument of preclinical AD and is the basis on which the clinical trials in preclinical AD are being conducted [18].

\section{"AD pathophysiological signature"}

Three approaches can be of use to search for signs of AD pathophysiology in individuals with a normal cognition. The gold-standard one is the post-mortem brain examination which can be used to directly assess regional $A \beta$ and tau pathology loads and provide a neuropathological diagnosis $[19,20]$. A limit of this method is that it only allows the study of subjects who died without any clinical impairment but it precludes the study of cognitive 
decline. Thus, rather than naming the concept "preclinical AD" in this type of study one could advocate the term "non-clinical AD pathology" or "silent AD pathology" as it is impossible to know if these subjects would have developed clinical symptoms if they have lived for a longer time. This neuropathological validation was performed in 4/55 (7.3\%) of this review's studies. The second method is the identification of a specific Mendelian autosomal dominant genetic mutation for familial AD (FAD). This allows studying preclinical early onset forms of AD as these mutations have a $100 \%$ penetrance so that all carriers will develop the disease. Moreover, the age of onset of symptoms in a mutation carrier is approximately the same as that of his parent. Cross sectional studies have been performed in these asymptomatic carriers to analyse the biomarker differences over time and to hypothesize their evolution [21]. A limitation is that the FAD population represents a minor fraction of all AD patients with differences in the expression, progression and pathophysiology of the disease such as the early age of onset. One out of the 55 studies (2\%) used this method in our review. The third way to identify the underlying $\mathrm{AD}$ physiopathology relies on the use of biomarkers. According to the IWG criteria, only some markers of AD such as CSF biomarkers (Aß, tau or phosphorylated tau) and amyloid and tau positon emission tomography (PET) but not MRI nor functional imaging are considered as pathophysiological markers [6]. In the NIA-AA criteria, brain (especially hippocampal) atrophy on MRI or hypometabolism on ${ }^{18}$ FDG-PET are also considered as suitable biomarkers to identify AD as they reflect a neurodegeneration pattern compatible with the disease [7]. In the present review the more restrictive IWG criteria were used so that each of the selected studies can be considered as relying on specific markers to assess an "AD pathophysiological signature" (CSF and/or amyloid and tau PET assessments). However, to date, there is no consensual biomarker-based method universally recognized to define "AD pathophysiological signature", such as prostate-specific antigen (PSA) values in prostate cancer or glucose values in diabetes [22]. In the studies reviewed herein, fourteen different definitions were applied for CSF biomarkers (CSF collection biomarkers assays, considered markers or panel of markers and cut-offs) and sixteen different definitions for amyloid PET (in terms of tracer, analytical methodology or threshold) out of the fifty biomarker based studies.

\section{Discussion and strategy for the standardization and harmonization of the Preclinical AD diagnostic and follow- up procedure.}

Following the problematic experience with the vastly heterogeneous application of prodromal "MCI" concepts to research studies and drug development programs in AD [23] and failures of recent large-scale trials aiming at slowing down progression of the disease in patients with mild to moderate $\mathrm{AD}[24,25]$, great interest has developed towards the earlier phase of the disease. At present, numerous clinical trials include prodromal AD participants with different definitions [23]. In view of the evolving paradigm change, preclinical $A D$, a concept that could provide a valuable early time window for therapeutic intervention, is under much scrutiny. The standardization of the neuropsychological and biomarker evaluation required for its diagnosis is an important challenge for future studies [26]. This is supported by converging evidence toward the possible efficacy of disease modifying drugs in the early clinical stage of AD [24, 27]. We propose that three issues should be addressed consistently in upcoming research on preclinical AD: the definition and diagnostic procedures of "normal cognition", "cognitive decline" and "AD physiopathological signature". In our review, these three 
determinants are largely heterogeneous which contributes among other, less modifiable factors such as geography of recruitment, to a substantial variability from one study population to the other. For instance, the ratio of stage 1 and 2 preclinical AD is of 78 and $22 \%$ respectively in one study [28] and of 21 and $79 \%$ in another one [29] limiting the generalizability of each study's findings. However, some homogeneity can also be evidenced. The CDR is the most commonly used tool used to define "normal cognition", and frequently used to assess "cognitive decline". Other endpoints are proposed that rely on various cognitive tests, diagnostic criteria for MCI or prodromal AD and since 2014, various composite cognitive scores. Compared to the CDR, these tests and composite scores offer the advantage of a finer delineation of the subtle cognitive changes that might occur many years before dementia is evidenced. On the other side, they are much more heterogeneous than the universally used CDR. Another issue with composite scores is their multiplicity. In the last two years, at least five different scores have been proposed relying on different methodologies (such as item response theory, or mean-to-standard deviation ratio) and including different tests [30-34]. Also, the use of subjective cognitive decline (SCD) was never considered as a marker of decline, even in studies focusing on memory complaints [15, 35-38] although it has been suggested that it might be a marker appearing late in the preclinical stage of the disease [9]. In fact, the main difference between "normal cognition" and "cognitive decline" could be drawn from differences in terms of relative risks (RR) to develop decline to milestones over time. For instance, an individual with SCD [39] should be considered as cognitively healthy since the RR of decline is low [40] and since the SCD condition is not specific of AD. The same can be said about psychomotor slowing and very mild executive changes which correspond to the "subtle cognitive changes" proposed by the NIA-AA. These symptoms arise many years before the dementia syndrome [9, 10, 38], are also non-specific and can be identified in other conditions such as mild vascular brain lesions [41] frontotemporal dementia [42] or even depression. On the other hand, when an individual has a low free recall in the free and cued selective reminding test (FCSRT) his risk to decline over the next years is high (>10 at 5 years) [43]. The specificity of the amnestic syndrome of the hippocampal type which is identifiable by this test allows for the classification of the subject in the clinical phase of $\mathrm{AD}$ (prodromal if it does not impact autonomy or dementia otherwise). This high risk profile and specificity for $\mathrm{AD}$, even at its prodromal stage were the reasons why this test was recommended in the first IWG research criteria for AD diagnosis [44] Likewise, the "subtle cognitive changes", namely attentional/psychomotor speed impairment, mild executive dysfunction should be operationalized as preclinical $\mathrm{AD}$ is more and more frequently studied. The chosen tests should be both the most frequently used ones by expert in the field and those which have demonstrated the best sensitivity to change over time in epidemiological studies on cognition in the elderly. The ten most frequently used tests in the 13 analysed cohorts are the Trail Making Test (TMT), Mini Mental State Evaluation (MMSE), Boston Naming Test, Verbal Fluency (animals), Clinical Dementia Rating scale (CDR), Logical Memory Test from the WMS-R, Rey Auditory Verbal Learning Test, Digit Span Forward and Backward from WMS-R, Digit Symbol Substitution Test (DSST) from WAIS-R and Verbal Fluency (letters). Performances below 1 standard deviations (SD) in cognitive tests and the individuals displaying these changes would still not be considered "cognitively impaired" in the absence of more specific symptoms. Studies conducted on the preclinical AD concept could be harmonized by 1) using tests to assess attention and psychomotor speed (such as the Digit Span Forward and DSST), executive functions (e.g. verbal fluencies, Trail making test), questionnaires to assess SCD, episodic memory (FCSRT), and global cognitive functioning (MMSE, CDR) (see table 5) and 2) by repeating these tests over time to identify "cognitive decline". On a schematic point of view, this aspecific/low risk first symptoms vs. high risk/specific impairments can be represented as in Fig 4 and might determine possible 
interventions. This appears to be the only way to establish prospectively which test performance(s) is (are) the best specific predictor(s) for the transition from the preclinical to the prodromal stage of the disease. Regarding the "AD pathophysiological signature", methods vary even more. This is most probably due to the recent development of these markers [45]. Of note for further studies, the recommendation to consider preclinical AD only in case of $A \beta$ AND tau positive markers points to the need to use either $A B$ and tau combinations of CSF biomarkers (12/50 studies with biomarkers in this review) or both A 3 and tau PET tracers (0 article in this review) [20]. Variability in the choice of cognitive tests and pathophysiological markers as determinants of preclinical $\mathrm{AD}$ was maximized when the authors of the studies made use of open-source databases and was reduced in studies focusing on cohorts that were analysed by individual research groups under the supervision of the same principal investigator [46, 47]. The ongoing innovation (e.g. the replacement of ${ }^{11} \mathrm{C}-\mathrm{PiB}$ by ${ }^{18}$ Fluor labelled amyloid ligands $[48,49]$ ) renders the process of standardization of biomarker results challenging. There are, however, international efforts to homogenize cognitive [50] and biomarker practice in research studies [51-53]. The specific value of different markers has also been studied [54] but no study combining all these markers with further post-mortem brain examination to determine the individual and combined added values of these marker has, to our knowledge, ever been conducted. The value of downstream topographical biomarkers of progression (brain atrophy on MRI and hypometabolism on ${ }^{18}$ FDG-PET) [48] as possible outcomes for decline should also be considered, notably in clinical trials [55]. Of course, it would be simplistic to consider preclinical AD as a homogeneous entity and the idea of proposing a "one size fits all" set of criteria may be problematic. But it is a necessary step to share results from different study groups. A unified definition of preclinical AD would of course not be definite but would evolve as different syndromic entities (eg fast vs. slow decliners) would emerge from ongoing studies. The fast paced innovation of biomarkers in the field also has to be considered. As new markers (such as blood based biomarkers) [56] are discovered and validated, their integration to the diagnostic algorithm of preclinical AD will have to be considered. In the end, a systems biology approach would be needed to propose a comprehensive set of definitions on as many preclinical AD variants as will be identified [57]. Of course, all these diagnostic processes rely on costly and invasive protocols that can, to date, only be proposed in the context of research projects in high income countries. This is reflected by the geographic location of the identified cohorts in this review and the low percentage of ethnic minorities among their participants. When a disease modifying treatment becomes available, the need to devise a pipeline of exams that is both safe and cost effective will be high. Specific neuro-economic studies should be conducted on the balance between the cost and adverse events due to a large scale screening for preclinical AD versus the long term benefit of early intervention at this stage of the disease.

One of the major limitation of this review is that it limits itself to the analysis of studies with more than one hundred participants. This choice was made empirically and the authors of this review recognize that many important insights for the field may be derived from studies with sizes below 100 subjects at this early exploratory and developmental period for the concept of preclinical AD. This choice was mainly made for one reason: to derive robust criteria for a disease or its risk factors you need an epidemiological study with a large number of participants and a long duration of follow-up, much like what the Framingham cohort brought to the cardiovascular field $[58,59]$. Our cut-off of 100 participants ensured that we identified some of the preclinical Alzheimer's field experienced centres with a total of 3854 individuals (sum of the total number of included participants in the latest published study of the 11 biomarker cohorts) with a mean (SEM) percentage of preclinical AD among them of 21.5 (2.2). Relatively to cohorts such as Framingham's, the effort to harmonize the definition of preclinical $\mathrm{AD}$ in order be able to share information among centres appears even more needed. 
In conclusion, even if total standardization of different markers of cognition and AD signature cannot be achieved, the community should agree on the use of some general tools in order to provide robust knowledge on the preclinical AD concept. For instance DSST, CDR, FCSRT for the neurocognitive evaluation, CSF biomarker evaluation adapted to reference analytical procedures such as the Gothenburg measurements [52] and amyloid PET SUVR

standardization for instance to a centiloid scale [51]. Also, an operationalized description in these studies of the various subtle cognitive changes occurring in preclinical AD (as proposed in table 5) could lead to a better understanding of the path to decline to be used as markers in clinical trials. As the first important step has been taken when the scientific community agreed on the general principles to define preclinical $\mathrm{AD}[1]$, the $\mathrm{AD}$ community must take the next step toward a unified procedure to diagnose this disease stage. 


\section{References}

[1] Dubois B, Hampel H, Feldman HH, Scheltens P, Aisen P, Andrieu S, et al. Preclinical Alzheimer's disease: Definition, natural history, and diagnostic criteria. Alzheimers Dement. 2016;12:292-323.

[2] Berg L, McKeel DW, Jr., Miller JP, Storandt M, Rubin EH, Morris JC, et al. Clinicopathologic studies in cognitively healthy aging and Alzheimer's disease: relation of histologic markers to dementia severity, age, sex, and apolipoprotein E genotype. Arch Neurol. 1998;55:326-35.

[3] Delaere P, Duyckaerts C, Masters C, Beyreuther K, Piette F, Hauw JJ. Large amounts of neocortical beta A4 deposits without neuritic plaques nor tangles in a psychometrically assessed, non-demented person. Neurosci Lett. 1990;116:87-93.

[4] Hubbard BM, Fenton GW, Anderson JM. A quantitative histological study of early clinical and preclinical Alzheimer's disease. Neuropathol Appl Neurobiol. 1990;16:111-21. [5] Storandt M, Grant EA, Miller JP, Morris JC. Longitudinal course and neuropathologic outcomes in original vs revised MCI and in pre-MCI. Neurology. 2006;67:467-73.

[6] Dubois B, Feldman HH, Jacova C, Hampel H, Molinuevo JL, Blennow K, et al. Advancing research diagnostic criteria for Alzheimer's disease: the IWG-2 criteria. The Lancet Neurology. 2014;13:614-29.

[7] Sperling RA, Aisen PS, Beckett LA, Bennett DA, Craft S, Fagan AM, et al. Toward defining the preclinical stages of Alzheimer's disease: recommendations from the National Institute on Aging-Alzheimer's Association workgroups on diagnostic guidelines for Alzheimer's disease. Alzheimers Dement. 2011;7:280-92.

[8] Tang EY, Harrison SL, Errington L, Gordon MF, Visser PJ, Novak G, et al. Current Developments in Dementia Risk Prediction Modelling: An Updated Systematic Review. PLoS One. 2015;10:e0136181.

[9] Amieva H, Le Goff M, Millet X, Orgogozo JM, Peres K, Barberger-Gateau P, et al. Prodromal Alzheimer's disease: successive emergence of the clinical symptoms. Ann Neurol. 2008;64:492-8.

[10] Amieva H, Mokri H, Le Goff M, Meillon C, Jacqmin-Gadda H, Foubert-Samier A, et al. Compensatory mechanisms in higher-educated subjects with Alzheimer's disease: a study of 20 years of cognitive decline. Brain. 2014;137:1167-75.

[11] Jack CR, Jr. PART and SNAP. Acta Neuropathol. 2014;128:773-6.

[12] Knopman DS, Jack CR, Jr., Wiste HJ, Weigand SD, Vemuri P, Lowe V, et al. Short-term clinical outcomes for stages of NIA-AA preclinical Alzheimer disease. Neurology.

2012;78:1576-82.

[13] Mormino EC, Betensky RA, Hedden T, Schultz AP, Amariglio RE, Rentz DM, et al. Synergistic effect of beta-amyloid and neurodegeneration on cognitive decline in clinically normal individuals. JAMA Neurol. 2014;71:1379-85.

[14] Mormino EC, Betensky RA, Hedden T, Schultz AP, Ward A, Huijbers W, et al. Amyloid and APOE epsilon4 interact to influence short-term decline in preclinical Alzheimer disease. Neurology. 2014;82:1760-7.

[15] Amariglio RE, Becker JA, Carmasin J, Wadsworth LP, Lorius N, Sullivan C, et al. Subjective cognitive complaints and amyloid burden in cognitively normal older individuals. Neuropsychologia. 2012;50:2880-6.

[16] Ayutyanont N, Langbaum JB, Hendrix SB, Chen K, Fleisher AS, Friesenhahn M, et al. The Alzheimer's prevention initiative composite cognitive test score: sample size estimates for the evaluation of preclinical Alzheimer's disease treatments in presenilin 1 E280A mutation carriers. The Journal of clinical psychiatry. 2014;75:652-60. 
[17] Sperling R, Mormino E, Johnson K. The evolution of preclinical Alzheimer's disease: implications for prevention trials. Neuron. 2014;84:608-22.

[18] Sperling RA, Rentz DM, Johnson KA, Karlawish J, Donohue M, Salmon DP, et al. The A4 study: stopping AD before symptoms begin? Sci Transl Med. 2014;6:228fs13.

[19] Jicha GA, Abner EL, Schmitt FA, Kryscio RJ, Riley KP, Cooper GE, et al. Preclinical AD Workgroup staging: pathological correlates and potential challenges. Neurobiol Aging. 2012;33:622 e1- e16.

[20] Hyman BT, Phelps CH, Beach TG, Bigio EH, Cairns NJ, Carrillo MC, et al. National Institute on Aging-Alzheimer's Association guidelines for the neuropathologic assessment of Alzheimer's disease. Alzheimers Dement. 2012;8:1-13.

[21] Bateman RJ, Xiong C, Benzinger TL, Fagan AM, Goate A, Fox NC, et al. Clinical and biomarker changes in dominantly inherited Alzheimer's disease. N Engl J Med.

2012;367:795-804.

[22] WHO. Definition, diagnosis and classification of diabetes mellitus and its complications.

Part 1: Diagnosis and classification of diabetes mellitus. Geneva1999.

[23] Hampel H, Lista S. Dementia: The rising global tide of cognitive impairment. Nat Rev

Neurol. 2016;12:131-2.

[24] Doody RS, Thomas RG, Farlow M, Iwatsubo T, Vellas B, Joffe S, et al. Phase 3 trials of solanezumab for mild-to-moderate Alzheimer's disease. N Engl J Med. 2014;370:311-21.

[25] Salloway S, Sperling R, Fox NC, Blennow K, Klunk W, Raskind M, et al. Two phase 3 trials of bapineuzumab in mild-to-moderate Alzheimer's disease. N Engl J Med.

2014;370:322-33.

[26] Khachaturian ZS, Mesulam MM, Mohs RC, Khachaturian AS. Toward a consensus recommendation for defining the asymptomatic-preclinical phases of putative Alzheimer's disease? Alzheimers Dement. 2016;12:213-5.

[27] Sevigny J, Chiao P, Bussière T, Weinreb PH, Williams L, Maier M, et al. The antibody aducanumab reduces A $\beta$ plaques in Alzheimer's disease. Nature. 2016;537:50-6.

[28] Brier MR, Thomas JB, Snyder AZ, Wang L, Fagan AM, Benzinger T, et al.

Unrecognized preclinical Alzheimer disease confounds rs-fcMRI studies of normal aging.

Neurology. 2014;83:1613-9.

[29] Fortea J, Vilaplana E, Alcolea D, Carmona-Iragui M, Sanchez-Saudinos MB, Sala I, et al. Cerebrospinal fluid beta-amyloid and phospho-tau biomarker interactions affecting brain structure in preclinical Alzheimer disease. Ann Neurol. 2014;76:223-30.

[30] Clark LR, Racine AM, Koscik RL, Okonkwo OC, Engelman CD, Carlsson CM, et al. Beta-amyloid and cognitive decline in late middle age: Findings from the Wisconsin Registry for Alzheimer's Prevention study. Alzheimers Dement. 2016;12:805-14.

[31] Donohue MC, Sperling RA, Salmon DP, Rentz DM, Raman R, Thomas RG, et al. The preclinical Alzheimer cognitive composite: measuring amyloid-related decline. JAMA Neurol. 2014;71:961-70.

[32] Langbaum JB, Hendrix SB, Ayutyanont N, Chen K, Fleisher AS, Shah RC, et al. An empirically derived composite cognitive test score with improved power to track and evaluate treatments for preclinical Alzheimer's disease. Alzheimers Dement. 2014;10:666-74.

[33] Lim YY, Maruff P, Pietrzak RH, Ames D, Ellis KA, Harrington K, et al. Effect of amyloid on memory and non-memory decline from preclinical to clinical Alzheimer's disease. Brain. 2014;137:221-31.

[34] Lim YY, Snyder PJ, Pietrzak RH, Ukiqi A, Villemagne VL, Ames D, et al. Sensitivity of composite scores to amyloid burden in preclinical Alzheimer's disease: Introducing the Zscores of Attention, Verbal fluency, and Episodic memory for Nondemented older adults composite score. Alzheimer's \& dementia. 2016;2:19-26. 
[35] Mielke MM, Wiste HJ, Weigand SD, Knopman DS, Lowe VJ, Roberts RO, et al. Indicators of amyloid burden in a population-based study of cognitively normal elderly. Neurology. 2012;79:1570-7.

[36] Pietrzak RH, Lim YY, Ames D, Harrington K, Restrepo C, Martins RN, et al.

Trajectories of memory decline in preclinical Alzheimer's disease: results from the Australian Imaging, Biomarkers and Lifestyle Flagship Study of ageing. Neurobiol Aging.

2015;36:1231-8.

[37] Pike KE, Ellis KA, Villemagne VL, Good N, Chetelat G, Ames D, et al. Cognition and beta-amyloid in preclinical Alzheimer's disease: data from the AIBL study. Neuropsychologia. 2011;49:2384-90.

[38] van Harten AC, Smits LL, Teunissen CE, Visser PJ, Koene T, Blankenstein MA, et al. Preclinical AD predicts decline in memory and executive functions in subjective complaints. Neurology. 2013;81:1409-16.

[39] Jessen F, Amariglio RE, van Boxtel M, Breteler M, Ceccaldi M, Chetelat G, et al. A conceptual framework for research on subjective cognitive decline in preclinical Alzheimer's disease. Alzheimers Dement. 2014.

[40] Mitchell AJ, Beaumont H, Ferguson D, Yadegarfar M, Stubbs B. Risk of dementia and mild cognitive impairment in older people with subjective memory complaints: meta-analysis. Acta psychiatrica Scandinavica. 2014;130:439-51.

[41] Jouvent E, Reyes S, De Guio F, Chabriat H. Reaction Time is a Marker of Early Cognitive and Behavioral Alterations in Pure Cerebral Small Vessel Disease. J Alzheimers Dis. 2015;47:413-9.

[42] Rohrer JD, Nicholas JM, Cash DM, van Swieten J, Dopper E, Jiskoot L, et al. Presymptomatic cognitive and neuroanatomical changes in genetic frontotemporal dementia in the Genetic Frontotemporal dementia Initiative (GENFI) study: a cross-sectional analysis. The Lancet Neurology. 2015;14:253-62.

[43] Di Stefano F, Epelbaum S, Coley N, Cantet C, Ousset PJ, Hampel H, et al. Prediction of Alzheimer's Disease Dementia: Data from the GuidAge Prevention Trial. J Alzheimers Dis. 2015;48:793-804.

[44] Dubois B, Feldman HH, Jacova C, Dekosky ST, Barberger-Gateau P, Cummings J, et al. Research criteria for the diagnosis of Alzheimer's disease: revising the NINCDS-ADRDA criteria. The Lancet Neurology. 2007;6:734-46.

[45] Hampel H, Frank R, Broich K, Teipel SJ, Katz RG, Hardy J, et al. Biomarkers for Alzheimer's disease: academic, industry and regulatory perspectives. Nat Rev Drug Discov. 2010;9:560-74.

[46] Morris JC, Roe CM, Grant EA, Head D, Storandt M, Goate AM, et al. Pittsburgh Compound B imaging and prediction of progression from cognitive normality to symptomatic Alzheimer disease. Archives of Neurology. 2009;66:1469-75.

[47] Morris JC, Roe CM, Xiong C, Fagan AM, Goate AM, Holtzman DM, et al. APOE predicts amyloid-beta but not tau Alzheimer pathology in cognitively normal aging. Annals of Neurology.67:122-31.

[48] Clark CM, Schneider JA, Bedell BJ, Beach TG, Bilker WB, Mintun MA, et al. Use of florbetapir-PET for imaging beta-amyloid pathology. JAMA. 2011;305:275-83.

[49] Rinne JO, Wong DF, Wolk DA, Leinonen V, Arnold SE, Buckley C, et al. [(18)F]Flutemetamol PET imaging and cortical biopsy histopathology for fibrillar amyloid beta detection in living subjects with normal pressure hydrocephalus: pooled analysis of four studies. Acta Neuropathol. 2012;124:833-45.

[50] Rabin LA, Smart CM, Crane PK, Amariglio RE, Berman LM, Boada M, et al. Subjective Cognitive Decline in Older Adults: An Overview of Self-Report Measures Used Across 19 International Research Studies. J Alzheimers Dis. 2015;48 Suppl 1:S63-86. 
[51] del Campo M, Mollenhauer B, Bertolotto A, Engelborghs S, Hampel H, Simonsen AH, et al. Recommendations to standardize preanalytical confounding factors in Alzheimer's and Parkinson's disease cerebrospinal fluid biomarkers: an update. Biomark Med. 2012;6:419-30. [52] Jagust WJ, Landau SM, Koeppe RA, Reiman EM, Chen K, Mathis CA, et al. The Alzheimer's Disease Neuroimaging Initiative 2 PET Core: 2015. Alzheimers Dement. 2015;11:757-71.

[53] Toledo JB, Zetterberg H, van Harten AC, Glodzik L, Martinez-Lage P, BocchioChiavetto L, et al. Alzheimer's disease cerebrospinal fluid biomarker in cognitively normal subjects. Brain. 2015;138:2701-15.

[54] Jack CR, Jr., Wiste HJ, Weigand SD, Knopman DS, Mielke MM, Vemuri P, et al. Different definitions of neurodegeneration produce similar amyloid/neurodegeneration biomarker group findings. Brain. 2015;138:3747-59.

[55] Dubois B, Chupin M, Hampel H, Lista S, Cavedo E, Croisile B, et al. Donepezil decreases annual rate of hippocampal atrophy in suspected prodromal Alzheimer's disease. Alzheimers Dement. 2015;11:1041-9.

[56] Hampel H, Lista S, Teipel SJ, Garaci F, Nistico R, Blennow K, et al. Perspective on future role of biological markers in clinical therapy trials of Alzheimer's disease: a long-range point of view beyond 2020. Biochem Pharmacol. 2014;88:426-49.

[57] Lista S, Khachaturian ZS, Rujescu D, Garaci F, Dubois B, Hampel H. Application of systems theory in longitudinal studies on the evolution and progression of Alzheimer's disease. In: Castrillo JI, Oliver SG, editors. Systems Biology of Alzheimer's Disease. New York: Springer Science+Business Media; 2016. p. 49-67.

[58] Chen G, Levy D. Contributions of the Framingham Heart Study to the Epidemiology of Coronary Heart Disease. JAMA cardiology. 2016;1:825-30.

[59] Mahmood SS, Levy D, Vasan RS, Wang TJ. The Framingham Heart Study and the epidemiology of cardiovascular disease: a historical perspective. Lancet. 2014;383:999-1008.

\section{Figures \& Tables}

Figure 1: PRISMA (2009) flow diagram of article selection.

Figure 2: Number of studies categorized by the cohort from which they are derived

Figure 3: Presentation of the different cognitive tests used in the thirteen cohorts

Figure 4: Schematic representation of Alzheimer's disease (AD) clinical spectrum compared to that of Fronto-temporal dementia and Lewy body dementia. The three horizontal lines indicate a change in state from totally asymptomatic preclinical state (lowest quadrant) to preclinical state with subtle cognitive changes to prodromal to dementia (upper quadrant). The initial "preclinical" phase of the disease is represented as a unique triangle encompassing all of the diseases to reflect the difficulty to clinically distinguish one entity from the next at this stage. The five smaller triangles each correspond to one affection. The "..." indicate that the model can be extended with other neurocognitive affections. In the prodromal phase they are well separated as clinical symptoms are often specific of one disease. At the dementia stage, the overlap between these triangles indicate the association of diverse symptoms obfuscating distinct diagnosis. AD physiopathological biomarker status (displayed by the continuity of the yellow dotted line and the yellow triangle) is considered positive from the totally asymptomatic preclinical state to the dementia stage. 
Table 1: Description of studies populations

Table 2: Studies methodology

Table 3: Cohorts collecting cognitive and AD pathophysiological markers data in asymptomatic individuals allowing the study of the preclinical AD concept

Table 4: Clinical trials in preclinical AD patients

Table 5: Proposed guidelines and nomenclature to operationalize Preclinical AD stages.

Supplemental Table 1: Detailed Description of studies populations

Supplemental Table 2: Detailed Studies methodology

Supplemental Table 3: Cognitive batteries performed in the different cohorts
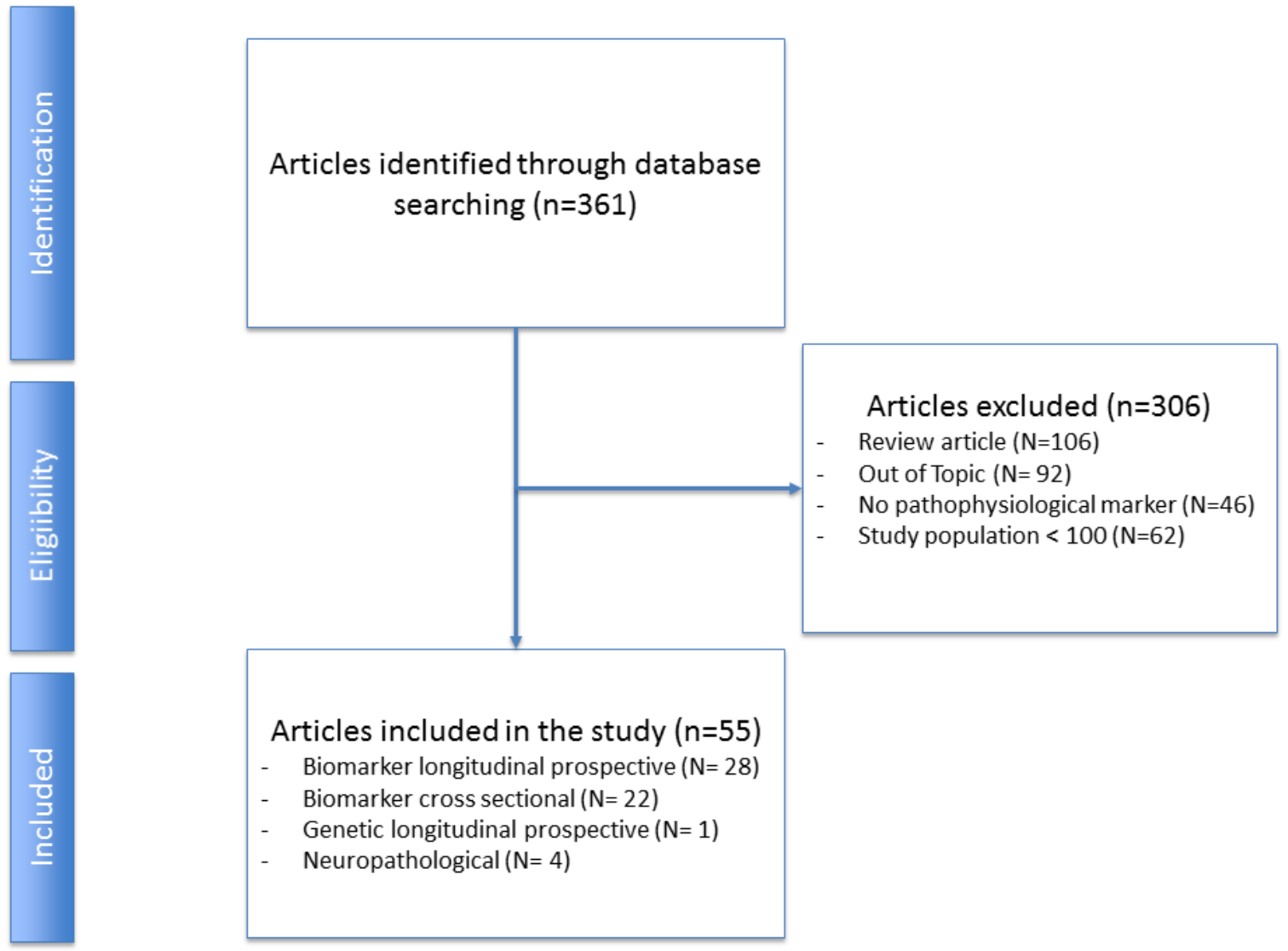

Figure 1 
Figure 2

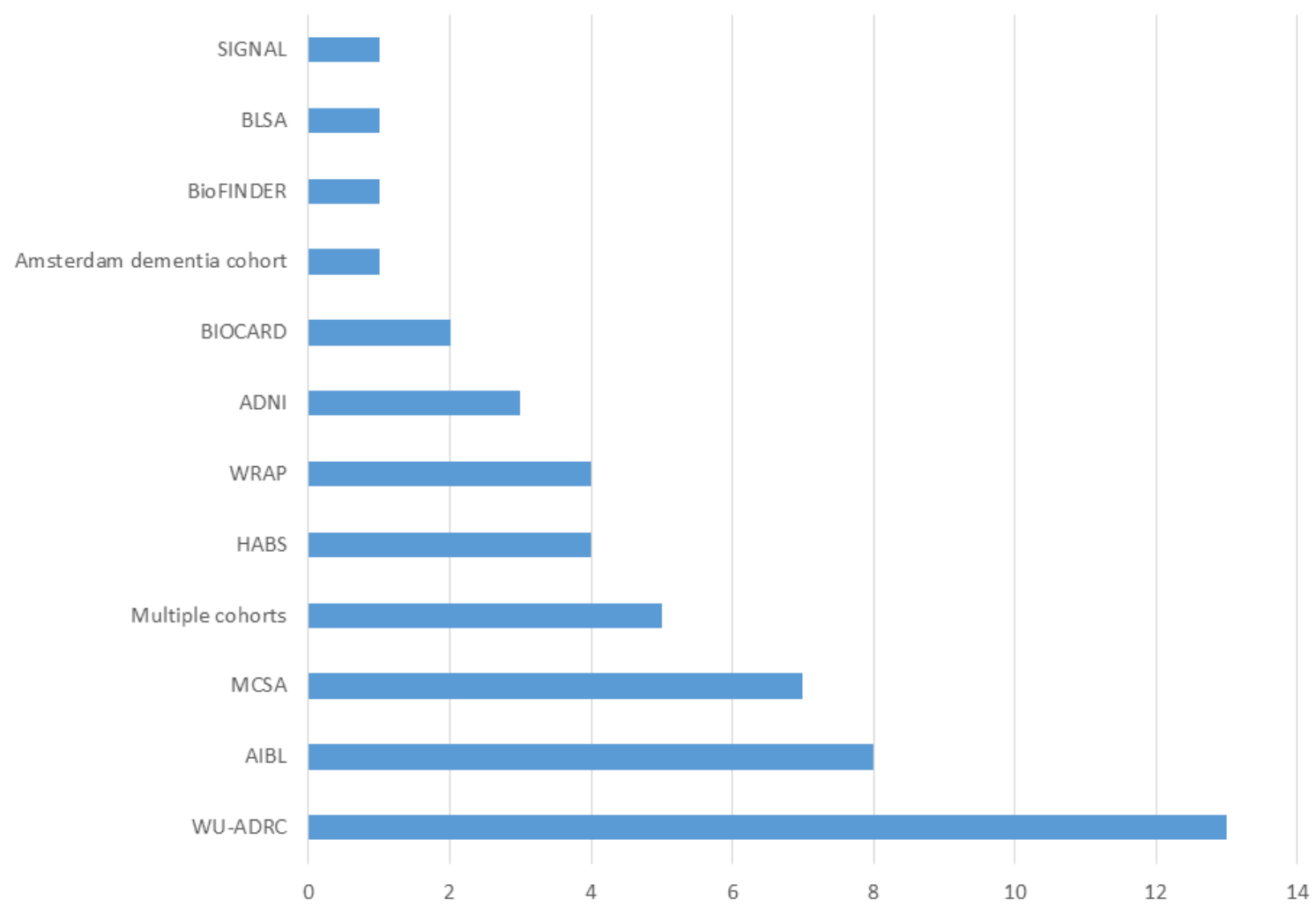

Figure 2 

Controlled Oral Word Frequency
Judgement of Line Orientation

Faces from WMS-III

Wisconsin Card Sort-64

WAIS-III working memory subtest Word list (CERAD)

Consortium to Establish a Registry for Alzheimer's Disease (CERAD) battery Wechsler Memory Scale-Revised (WMS-R) WMS Mental Control

Wide Range Achievement Reading Test (WART) Wechsler Abbreviated Scale of Intelligence (WASI) WAIS Information Wechsler Adult Intelligence Scale-Revised (WAIS-R) Visual Object and Space Perception Battery Visual Association Test Visual Form Discrimination Test Six-trial Selective Reminding Test Number Cancellation Test

Multiple choice vocabulary test (SRB-1) Montreal Cognitive Assessment (MoCA) Memory Functioning Questionnaire (subjective cognitive complaint) Letter Number Sequencing Free and Cued Selective Reminding Test (FCSRT) Cube Copying Cogstate Battery Card Rotation Test Cambridge Evaluation for Global Cognitive Decline A Quick Test of Cognitive Speed (AQT) Word recall (immediate and delayed) form ADAS-Co

American National Adult Reading Test Alzheimer's Disease Asessment Scale (ADAS-Cog) Benton Visual Retention Test Stroop Test Rey-Osterreith Complex Figure E-Cog scale (Everyday cognition) Months Backwards Clock Drawing California Verbal Learning Test (CVLT) Paired Associated Memory Test of the WMS-R Block Design from the WAIS-R

Rey Aud itory Verbal Learning Test Logical Memory Test from WMS-R Digit Symbol Substitution Test (DSST) from WAIS-R Verbal Fluency (letters)

Digit Span Forward and Backward from WMS-R Clinical Dementia Rating scale (CDR) Verbal Fluency (animals) Boston NamingTest

Mini Mental State Evaluation (MMSE) Trail Making Test (TMT)

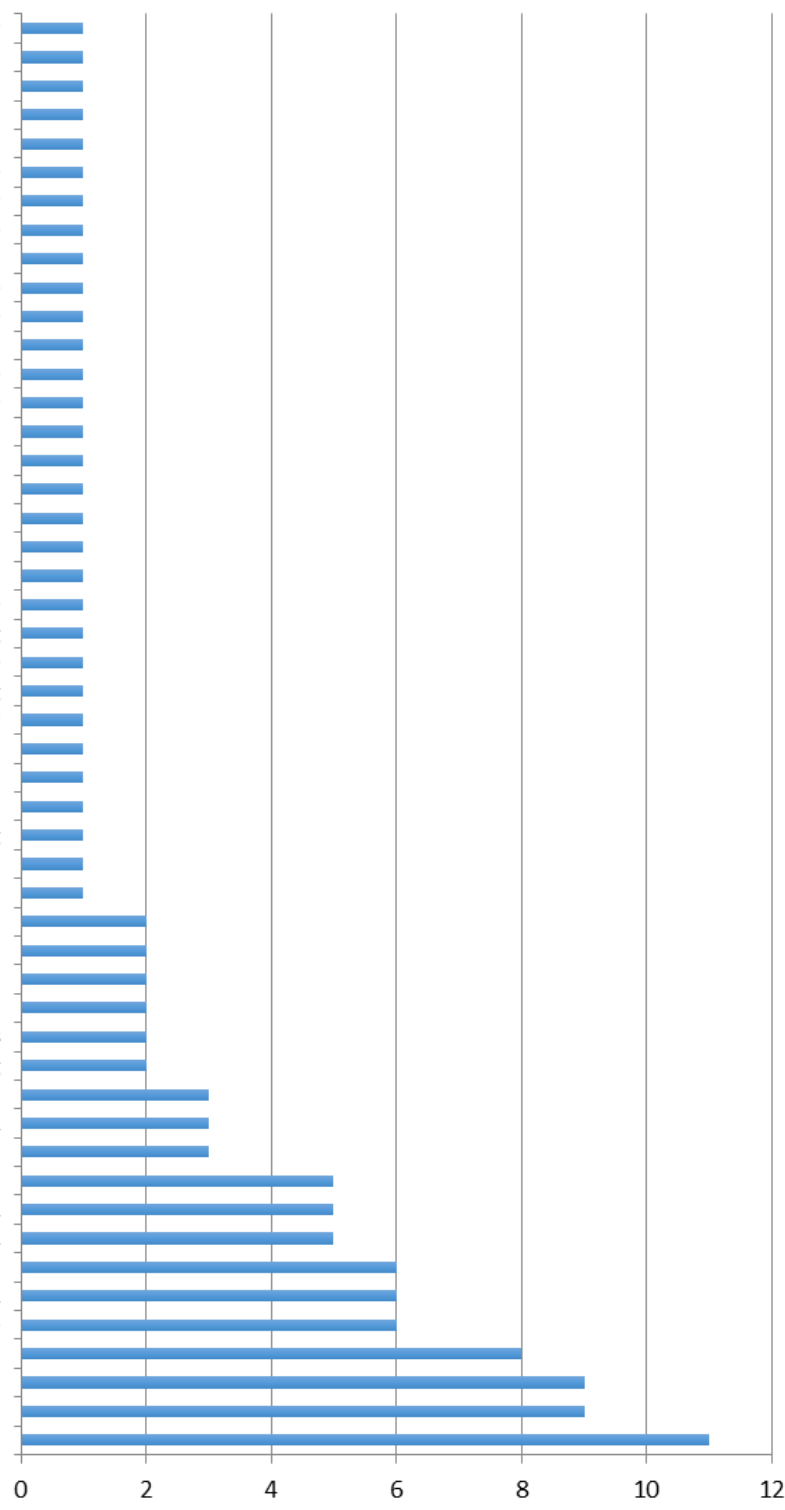

Figure 3 


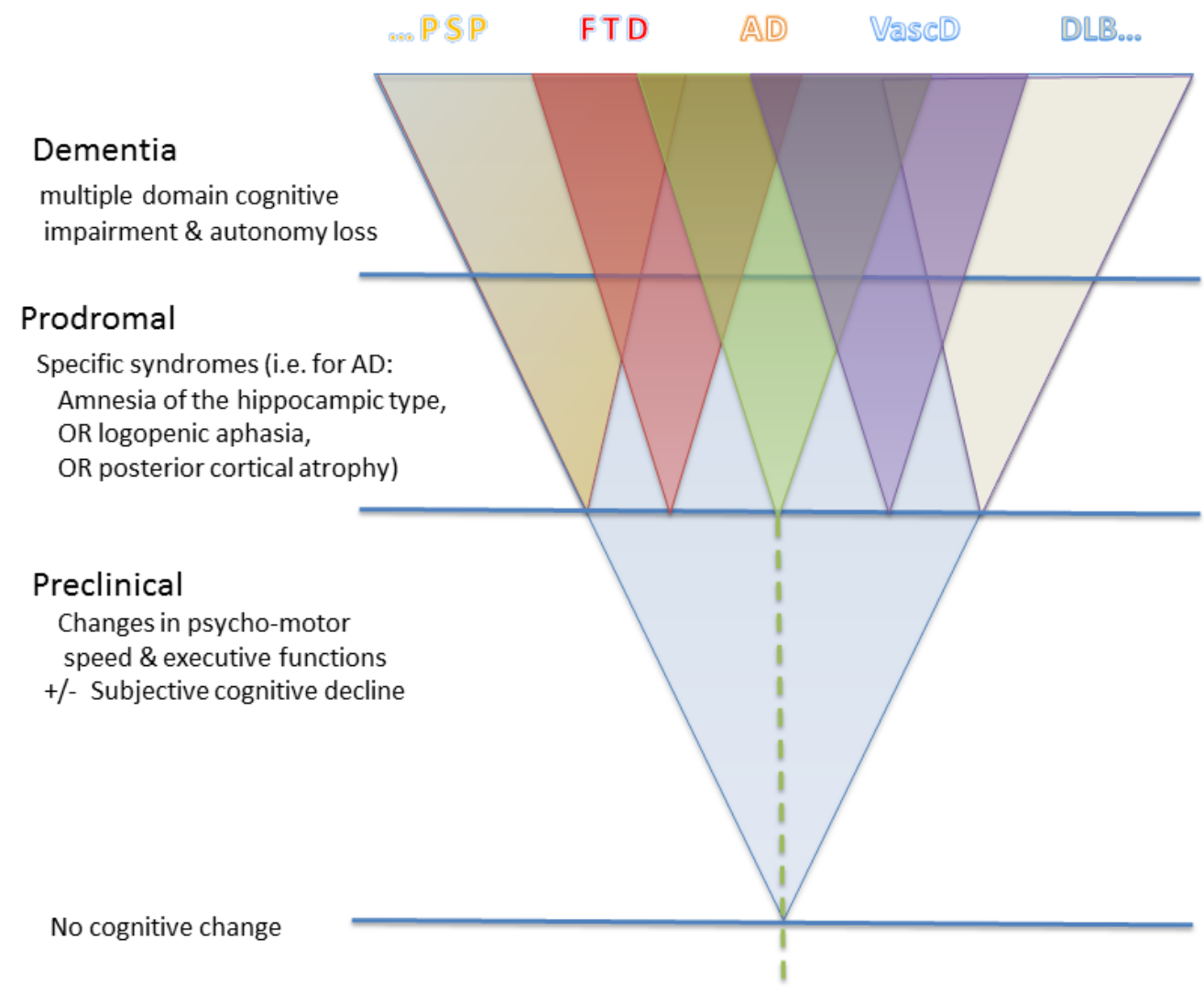

$\underset{m}{\exists}$

Figure 4 


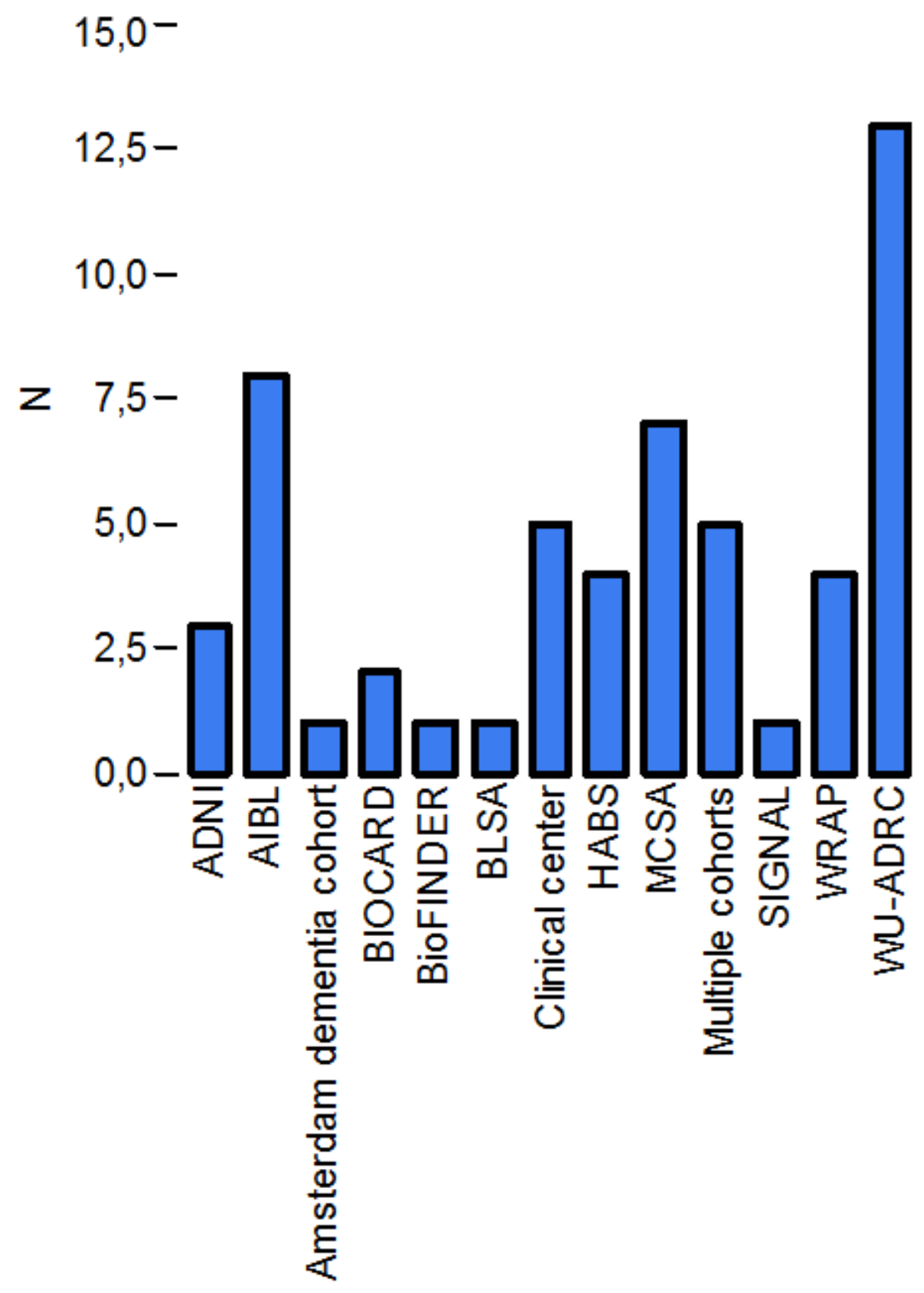

Cohort

Suppl Figure 1 
TABLE 1 STUDIES POPULATIONS

$N(\%)$ or Mean (SEM)

\begin{tabular}{ccc}
$\begin{array}{c}\text { Cross Sectional } \\
\text { studies }\end{array}$ & $\begin{array}{c}\text { Longitudinal } \\
\text { Studies } \\
N=22\end{array}$ & $\begin{array}{c}\text { Neuropathological } \\
\text { studies } \\
N=4\end{array}$ \\
\hline $13(59)$ & $21(72)$ & $0(0)$ \\
$331.5(48.2)$ & $261.1(42)$ & $866(550)^{* *}$ \\
$71.4(1,6)$ & $68.1(1,3)$ & $78.6(3.3)$ \\
$158.4(22.2)$ & $164.3(20.2)$ & $184(110.3)$ \\
$55.8(3.6)$ & $65.2(3.2)$ & $58(8.2)$ \\
$83.6(12.3)$ & $65.4(11.2)$ & $111.3(68.6)$ \\
\hline $27.3(1.5)$ & $26.4(1.4)$ & $33.7(5)$ \\
$8(36.4)$ & $7(24.1)$ & $1(25)$
\end{tabular}

NIA-AA PC AD Criteria [1] or [2]
$N$ of studies using the conceptual framework (\%)
Stage I*

SNAP percentage of total population

Stage $I^{*}$

APOE4 percentage of Total population

APOE4 percentage of $P C A D$

\begin{tabular}{|cc|c|}
\hline $54(5.2)$ & $53(5.6)$ & 24 \\
\hline $41.4(6.3)$ & $43.6(6.3)$ & 28 \\
\hline $20.4(1.7)$ & $21.5(1.6)$ & 10 \\
\hline $31(2)$ & $34.8(1.5)$ & $29(1)$ \\
$50.7(3.7)$ & $44.1(3.3)$ & $32.1(0.9)$ \\
\hline
\end{tabular}

$* \%$ of PC AD. Stage III was only rarely applied (i.e. in 5 of 8 cross sectional studies and 4 of

7 longitudinal studies using this terminology) and so was not included in the table. ** One large study did not give any detail on the number of preclinical AD which accounts for the discrepancy between the Study population total size and the rest of the table figures for the Neuropathology column. PC AD = Preclinical Alzheimer's disease, HC: Healthy control.

[1] Sperling RA, Aisen PS, Beckett LA, Bennett DA, Craft S, Fagan AM, et al. Toward defining the preclinical stages of Alzheimer's disease: recommendations from the National Institute on Aging-Alzheimer's Association workgroups on diagnostic guidelines for Alzheimer's disease. Alzheimers Dement. 2011;7:280-92.

[2] Jicha GA, Abner EL, Schmitt FA, Kryscio RJ, Riley KP, Cooper GE, et al. Preclinical AD Workgroup staging: pathological correlates and potential challenges. Neurobiol Aging. 2012;33:622 e1- e16. 
TABLE 2. STUDIES METHODOLOGY

\begin{tabular}{|c|c|c|c|}
\hline$N(\%)$ & $\begin{array}{c}\text { Cross } \\
\text { Sectional } \\
\text { (CS) } \\
\text { Studies } \\
N=22\end{array}$ & $\begin{array}{l}\text { Longitudinal }(L) \\
\text { Studies } \\
N=29\end{array}$ & $\begin{array}{c}\text { Neuropathology }(N) \\
\text { Studies } \\
N=4\end{array}$ \\
\hline $\begin{array}{l}\text { Criteria for } \\
\text { Normal } \\
\text { Cognition }\end{array}$ & $\begin{array}{l}\text { Available in } \\
\mathrm{N}=20 \\
\text { (90.9\% of CS } \\
\text { studies) }\end{array}$ & $\begin{array}{c}\text { Available in } \mathrm{N}= \\
27 \\
\text { (93.1\% of } \mathrm{L} \\
\text { studies) }\end{array}$ & $\begin{array}{l}\text { Available in } \mathrm{N}=3 \\
\text { (75\% of } \mathrm{N} \text { studies) }\end{array}$ \\
\hline$C D R$ & $4(20)$ & $8(29.6)$ & $1(33.3)$ \\
\hline$C D R+$ & $4(30)$ & $4(14.8)$ & 0 \\
\hline Cognitive battery & $8(40)$ & $4(14.8)$ & $1(33.3)$ \\
\hline MMSE & $2(10)$ & $10(37.1)$ & $1(33.3)$ \\
\hline Clinical Consensus & 0 & $1(3.7)$ & 0 \\
\hline $\begin{array}{l}\text { Criteria for } \\
\text { Cognitive } \\
\text { Decline }\end{array}$ & $\begin{array}{c}\text { Not } \\
\text { applicable }\end{array}$ & $\begin{array}{c}\text { Available in } \mathrm{N}= \\
27 \\
\text { (93.1\% of } \mathrm{L} \\
\text { studies) }\end{array}$ & $\begin{array}{l}\text { Available in } N=1 \\
(25 \% \text { of } N \text { studies) }\end{array}$ \\
\hline$C D R$ & - & $6(22.3)$ & $1(100)$ \\
\hline$C D R+$ & - & $1(3.7)$ & 0 \\
\hline Cognitive battery & - & $3(11.1)$ & 0 \\
\hline Composite scores & - & $12(44.4)$ & 0 \\
\hline Clinical consensus & - & $5(18.5)$ & 0 \\
\hline \multicolumn{4}{|l|}{$\begin{array}{l}\text { Criteria for } A D \\
\text { pathophysiological } \\
\text { signature }\end{array}$} \\
\hline CSF biomarkers & $9(40.9)$ & $7(24.2)$ & 0 \\
\hline Amyloid PET & $11(50)$ & $17(58.6)$ & 0 \\
\hline Both & $2(9.1)$ & $4(13.8)$ & 0 \\
\hline Mutation & 0 & $1(3.4)$ & 0 \\
\hline Neuropathology & 0 & 0 & $4(100)$ \\
\hline
\end{tabular}

CDR: Clinical dementia rating scale, CDR+: association of a Clinical dementia rating scale and at least one other neuropsychological test, Cognitive battery: association of at least two cognitive test, Clinical consensus: Adjudication by an expert committee of clinicians into one of three categories (normal cognition, mild cognitive impairment or dementia), CSF biomarkers: use of either cerebrospinal fluid $A ß_{1-42}$, tau, phosphorylated tau concentrations or a combination of these markers. Amyloid PET : use of either PIB, florbetapir or flutemetamol. Mutation : evidence of the presenilin 1 (PSEN1) E280A mutation. Neuropathology: Neuropathological evidence of Alzheimer's disease. 
Table 3.

\begin{tabular}{|c|c|c|c|c|c|c|c|c|c|c|c|c|c|c|c|c|}
\hline $\begin{array}{c}\text { Cohor } \\
t\end{array}$ & $\mathbf{N}$ & $\begin{array}{l}\text { T } \\
y \\
p \\
\text { e }\end{array}$ & $\begin{array}{l}\text { D } \\
\text { e } \\
\text { si } \\
\text { g } \\
\text { n }\end{array}$ & $\begin{array}{c}\text { Cou } \\
\text { ntry } \\
\text { /sta } \\
\text { te }\end{array}$ & $\begin{array}{c}\text { Eth } \\
\text { nici } \\
\text { ty } \\
/ \mathbf{m} \\
\text { ino } \\
\text { riti } \\
\text { es }\end{array}$ & $\begin{array}{l}\text { Po } \\
\text { pul } \\
\text { ati } \\
\text { on } \\
\text { (M } \\
\text { /F; }\end{array}$ & $\begin{array}{c}\text { Age } \\
\text { (Me } \\
\text { an } \\
+/- \\
\text { SD } \\
\text { or } \\
\text { Ran } \\
\text { ge) }\end{array}$ & $\begin{array}{l}\text { Cognit } \\
\text { ive } \\
\text { Integr } \\
\text { ity } \\
\text { Criteri } \\
\text { a }\end{array}$ & $\begin{array}{c}\text { Nps } \\
\text { y } \\
\text { Batt } \\
\text { ery }\end{array}$ & $\begin{array}{c}\text { CS } \\
\text { F }\end{array}$ & $\begin{array}{c}\text { MR } \\
\text { I } \\
\text { se } \\
\text { qu } \\
\text { en } \\
\text { ces }\end{array}$ & $\begin{array}{c}18 \\
\text { FD } \\
\text { G- } \\
\text { PE } \\
\text { T }\end{array}$ & $\begin{array}{l}\text { Amy } \\
\text { loid } \\
\text { PET }\end{array}$ & $\begin{array}{l}\text { Blo } \\
\text { od }\end{array}$ & $\begin{array}{c}\text { Other } \\
\text { Biom } \\
\text { arker } \\
\quad \text { s }\end{array}$ & $\begin{array}{c}\text { Cohor } \\
\text { t } \\
\text { Refer } \\
\text { ence }\end{array}$ \\
\hline $\begin{array}{c}\text { ADNI } \\
\text { 1-GO- } \\
2\end{array}$ & $\begin{array}{c}14 \\
5\end{array}$ & $\mathrm{R}$ & $\begin{array}{l}\text { M } \\
\mathrm{u} \\
\mathrm{lt} \\
\mathrm{i}\end{array}$ & USA & $\begin{array}{c}\text { Ca } \\
\text { uca } \\
\text { sia } \\
\text { ns } \\
93 \\
\%\end{array}$ & $\begin{array}{l}\mathrm{M} / \\
\mathrm{F}= \\
58 \\
/ 4 \\
2\end{array}$ & $\begin{array}{l}55- \\
90\end{array}$ & $\begin{array}{c}\text { MMSE } \\
>24 \text {, } \\
\text { CDR=0 } \\
\text {, No- } \\
\text { depres } \\
\text { sed, } \\
\text { MCI } \\
\text { nor } \\
\text { demen } \\
\text { ted }\end{array}$ & Yes & $\begin{array}{c}\mathrm{Su} \\
\mathrm{bsa} \\
\mathrm{mp} \\
\mathrm{le}\end{array}$ & Yes & $\begin{array}{c}\mathrm{Su} \\
\mathrm{bsa} \\
\mathrm{mp} \\
\mathrm{le}\end{array}$ & $\begin{array}{c}\text { Subs } \\
\text { amp } \\
\text { le }\end{array}$ & Yes & NA & $\begin{array}{c}\text { Weine } \\
\mathrm{r} \text { et al. } \\
2010\end{array}$ \\
\hline $\begin{array}{c}\text { AMST } \\
\text { ERDA } \\
\text { M } \\
\text { Deme } \\
\text { ntia } \\
\text { Cohor } \\
\text { t } \\
\end{array}$ & $\begin{array}{c}13 \\
2\end{array}$ & C & $\begin{array}{l}\mathrm{M} \\
\mathrm{o} \\
\mathrm{n} \\
\mathrm{o}\end{array}$ & $\mathrm{NL}$ & $\begin{array}{c}\text { Not } \\
\text { me } \\
\text { nti } \\
\text { on } \\
\text { ne } \\
\text { d }\end{array}$ & $\begin{array}{l}\mathrm{M} / \\
\mathrm{F}= \\
56 \\
/ 4 \\
4\end{array}$ & $\begin{array}{l}64 \\
+/- \\
10\end{array}$ & $\begin{array}{c}\text { No CI } \\
\text { based } \\
\text { on a } \\
\text { NRPSY } \\
\text { Batter } \\
y\end{array}$ & Yes & $\begin{array}{c}\mathrm{Su} \\
\mathrm{bsa} \\
\mathrm{mp} \\
\mathrm{le}\end{array}$ & $\begin{array}{c}\mathrm{Su} \\
\mathrm{bsa} \\
\mathrm{mp} \\
\mathrm{le}\end{array}$ & $\begin{array}{c}\text { Su } \\
\text { bsa } \\
\text { mp } \\
\text { le }\end{array}$ & $\begin{array}{c}\text { Subs } \\
\text { amp } \\
\text { le }\end{array}$ & $\begin{array}{c}\mathrm{Su} \\
\mathrm{bsa} \\
\mathrm{mp} \\
\mathrm{le}\end{array}$ & $\begin{array}{c}\text { EEG } \\
\text { Subsa } \\
\text { mple }\end{array}$ & $\begin{array}{l}\text { van } \\
\text { der } \\
\text { Flier } \\
\text { et al. } \\
2014\end{array}$ \\
\hline $\begin{array}{c}\text { AIBL } \\
\text { study } \\
\text { (Aust } \\
\text { ralian } \\
\text { Imagi } \\
\text { ng, } \\
\text { Biom } \\
\text { arker } \\
\text { s and } \\
\text { Lifest } \\
\text { yle } \\
\text { study } \\
\text { ) }\end{array}$ & $\begin{array}{c}42 \\
3\end{array}$ & $\mathrm{R}$ & $\begin{array}{c}\mathrm{M} \\
\mathrm{u} \\
\mathrm{lt} \\
\mathrm{i}\end{array}$ & $\begin{array}{l}\text { Aust } \\
\text { ralia }\end{array}$ & $\begin{array}{c}\text { Not } \\
\text { me } \\
\text { nti } \\
\text { on } \\
\text { ne } \\
\text { d }\end{array}$ & $\begin{array}{l}\mathrm{M} / \\
\mathrm{F}= \\
42 \\
/ 5 \\
8\end{array}$ & $\begin{array}{c}70 \\
+/- \\
7\end{array}$ & $\begin{array}{c}\text { No CI } \\
\text { based } \\
\text { on a } \\
\text { NRPSY } \\
\text { Batter } \\
y\end{array}$ & Yes & $\begin{array}{c}\mathrm{Su} \\
\mathrm{bsa} \\
\mathrm{mp} \\
\mathrm{le}\end{array}$ & Yes & Yes & Yes & Yes & $\begin{array}{c}\text { EEG } \\
\text { Subsa } \\
\text { mple }\end{array}$ & $\begin{array}{c}\text { Ellis } \\
2009\end{array}$ \\
\hline $\begin{array}{l}\text { BIOC } \\
\text { ARD } \\
\text { (Pros } \\
\text { pectiv } \\
\text { e } \\
\text { Study } \\
\text { of } \\
\text { Bioma } \\
\text { rkers } \\
\text { for } \\
\text { Older } \\
\text { Contr } \\
\text { ols at } \\
\text { Risk } \\
\text { for } \\
\text { Alzhei } \\
\text { mer's } \\
\text { Diseas } \\
\text { e) }\end{array}$ & $\begin{array}{c}30 \\
2\end{array}$ & $\mathrm{R}$ & $\begin{array}{l}\mathrm{M} \\
\mathrm{o} \\
\mathrm{n} \\
\mathrm{o}\end{array}$ & $\begin{array}{l}\text { USA, } \\
\text { MD }\end{array}$ & $\begin{array}{c}\text { Not } \\
\text { me } \\
\text { nti } \\
\text { on } \\
\text { ne } \\
\text { d }\end{array}$ & $\begin{array}{l}\text { M/ } \\
\text { F : } \\
41 \\
/ 5 \\
9\end{array}$ & $\begin{array}{l}\text { Mid } \\
\text { dle- } \\
\text { age }\end{array}$ & $\begin{array}{c}\text { Mattis } \\
\text { Demen } \\
\text { tia } \\
\text { Rating } \\
\text { Scale, } \\
\text { Buschk } \\
\text { e } \\
\text { Selecti } \\
\text { ve } \\
\text { Remin } \\
\text { ding } \\
\text { Test } \\
\text { (Busch } \\
\text { ke, } \\
\text { 1973), } \\
\text { and } \\
\text { Wechsl } \\
\text { er } \\
\text { Memor } \\
\text { y } \\
\text { Scale } \\
- \\
\text { Revise } \\
\text { d } \\
\text { (WMS- } \\
\text { R; } \\
\text { Wechsl } \\
\text { er, } \\
\text { 1987) } \\
\text { perfor } \\
\text { mance } \\
\text { within } \\
\text { the }\end{array}$ & Yes & Yes & Yes & No & $\begin{array}{c}\text { Subs } \\
\text { amp } \\
\text { le }\end{array}$ & Yes & $\begin{array}{c}\text { postm } \\
\text { ortem } \\
\text { neuro } \\
\text { patho } \\
\text { logic } \\
\text { evalu } \\
\text { ations } \\
\text { in a } \\
\text { subsa } \\
\text { mle }\end{array}$ & $\begin{array}{c}\text { Green } \\
\text { wood } \\
\text { et al. } \\
2005\end{array}$ \\
\hline
\end{tabular}




\begin{tabular}{|c|c|c|c|c|c|c|c|c|c|c|c|c|c|c|c|c|}
\hline & & & & & & & & $\begin{array}{c}\text { normal } \\
\text { age- } \\
\text { related } \\
\text { range } \\
\text { of } \\
\text { scores } \\
\end{array}$ & & & & & & & & \\
\hline $\begin{array}{c}\text { BioFI } \\
\text { NDER } \\
\text { (Biom } \\
\text { arkers } \\
\text { For } \\
\text { Identi } \\
\text { fying } \\
\text { Neuro } \\
\text { degen } \\
\text { erativ } \\
\text { e } \\
\text { Disor } \\
\text { ders } \\
\text { Early } \\
\text { and } \\
\text { Reliab } \\
\text { ly } \\
\text { cognit } \\
\text { ively } \\
\text { health } \\
\text { y } \\
\text { cohort } \\
\text { ) }\end{array}$ & $\begin{array}{c}35 \\
2\end{array}$ & $\mathrm{R}$ & $\begin{array}{l}\text { M } \\
u \\
\text { lt } \\
i\end{array}$ & $\begin{array}{c}\text { Swede } \\
\mathrm{n}\end{array}$ & $\begin{array}{c}\text { Not } \\
\text { ment } \\
\text { ionne } \\
\text { d }\end{array}$ & $\begin{array}{c}\text { M/F: } \\
46 / 5 \\
4\end{array}$ & $>60$ & $\begin{array}{c}\text { MMSE } \\
28-30 \\
\text { at } \\
\text { screeni } \\
\text { ng visit }\end{array}$ & Yes & Yes & Yes & No & Yes & Yes & $\begin{array}{l}\text { Tau } \\
\text { PET }\end{array}$ & $\begin{array}{l}\frac{\text { http:// }}{\text { biofind }} \\
\frac{\text { er.se/b }}{\text { iofinde }} \\
\frac{\underline{r \text { coho }}}{\text { rts/co }} \\
\frac{\text { gnitive }}{\text { ly- }} \\
\frac{\underline{\text { health }}}{\mathrm{y}-} \\
\frac{\text { elderly }}{L}\end{array}$ \\
\hline $\begin{array}{c}\text { BLSA } \\
\text { (Balti } \\
\text { more } \\
\text { longit } \\
\text { udina } \\
\text { l } \\
\text { study } \\
\text { of } \\
\text { Aging } \\
\text { J }\end{array}$ & $\begin{array}{c}10 \\
4\end{array}$ & $\mathrm{R}$ & $\begin{array}{l}\mathrm{M} \\
\mathrm{o} \\
\mathrm{n} \\
\mathrm{o}\end{array}$ & $\begin{array}{l}\text { USA, } \\
\text { MD. }\end{array}$ & $\begin{array}{c}73 . \\
6 \% \\
\text { Ca } \\
\text { uca } \\
\text { sia } \\
\text { ns }\end{array}$ & $\begin{array}{c}\text { M/ } \\
\text { F: } \\
50 . \\
5 / \\
49 . \\
5\end{array}$ & $\begin{array}{c}\text { Mea } \\
\mathrm{n} \\
77.3 \\
\text { year } \\
\mathrm{s}\end{array}$ & $\begin{array}{c}\text { No } \\
\text { MCI or } \\
\text { demen } \\
\text { tia by } \\
\text { clinical } \\
\text { evalua } \\
\text { tion } \\
\text { (i.e. No } \\
\text { substa } \\
\text { ntial CI } \\
\text { based } \\
\text { on } \\
\text { mental } \\
\text { status } \\
\text { screeni } \\
\text { ng } \\
\text { tests) }\end{array}$ & Yes & No & Yes & No & Yes & Yes & No & $\begin{array}{l}\frac{\text { https:/ }}{\text { Lwww. }} \\
\underline{\text { blsa.ni }} \\
\text { h.gov/ }\end{array}$ \\
\hline $\begin{array}{c}\text { HABS } \\
\text { (Harv } \\
\text { ard } \\
\text { Aging } \\
\text { Brain } \\
\text { Study } \\
\text { ) }\end{array}$ & $\begin{array}{c}27 \\
5\end{array}$ & $\mathrm{R}$ & $\begin{array}{l}\mathrm{M} \\
\mathrm{o} \\
\mathrm{n} \\
\mathrm{o}\end{array}$ & $\begin{array}{l}\text { USA, } \\
\text { MA }\end{array}$ & $\begin{array}{c}81 \\
\% \\
\text { Ca } \\
\text { uca } \\
\text { sia } \\
\text { ns }\end{array}$ & $\begin{array}{l}\text { M/ } \\
\text { F: } \\
41 \\
/ 5 \\
9\end{array}$ & $\begin{array}{c}62- \\
90\end{array}$ & $\begin{array}{c}\text { GDS }<1 \\
1, \\
\text { CDR=0 } \\
\text { MMSE } \\
>25 \\
\text { and } \\
\text { Norma } \\
\text { l } \\
\text { Perfor } \\
\text { mance } \\
\text { s at } \\
\text { Logical } \\
\text { Memor } \\
y \\
\text { delaye } \\
d \\
\text { recall }\end{array}$ & Yes & $\begin{array}{c}\text { Su } \\
\text { bsa } \\
\text { mp } \\
\text { le }\end{array}$ & Yes & Yes & Yes & Yes & NA & $\begin{array}{c}\text { Dagley } \\
\text { A } \\
2015\end{array}$ \\
\hline $\begin{array}{c}\text { MCSA } \\
\text { (May } \\
\text { o } \\
\text { Clinic } \\
\text { Study } \\
\text { of } \\
\text { Aging }\end{array}$ & $\begin{array}{l}13 \\
31\end{array}$ & $\mathrm{R}$ & $\begin{array}{l}\mathrm{M} \\
\mathrm{o} \\
\mathrm{n} \\
\mathrm{o}\end{array}$ & $\begin{array}{l}\text { USA, } \\
\text { MN }\end{array}$ & $\begin{array}{c}98, \\
6 \% \\
\mathrm{Ca} \\
\text { uca } \\
\text { sia } \\
\text { ns }\end{array}$ & $\begin{array}{c}\text { M/ } \\
\text { F : } \\
46 \\
/ 5 \\
4\end{array}$ & $\begin{array}{c}70- \\
90 \\
\text { year } \\
\text { s }\end{array}$ & $\begin{array}{c}\text { CDR } \\
=0 ; \\
\text { Norm } \\
\text { al } \\
\text { functio } \\
\text { nal } \\
\text { status ; }\end{array}$ & Yes & No & No & No & No & Yes & No & $\begin{array}{c}\text { Robert } \\
\text { s et al., } \\
2008 \\
\text { and } \\
2012\end{array}$ \\
\hline
\end{tabular}




\begin{tabular}{|c|c|c|c|c|c|c|c|c|c|c|c|c|c|c|c|c|}
\hline ) & & & & & & & & $\begin{array}{c}\text { NRPSY } \\
\text { testing } \\
\text { within } \\
\text { normal } \\
\text { limits }\end{array}$ & & & & & & & & \\
\hline $\begin{array}{c}\text { NACC } \\
\text { (Nati } \\
\text { onal } \\
\text { Alzhe } \\
\text { imer' } \\
\text { s } \\
\text { Coord } \\
\text { inatin } \\
\text { g } \\
\text { Cente } \\
\text { r } \\
\text { datab } \\
\text { ase) }\end{array}$ & - & $\mathrm{R}$ & $\begin{array}{l}\mathrm{M} \\
\mathrm{o} \\
\mathrm{n} \\
\mathrm{o}\end{array}$ & USA & $\begin{array}{c}80 \\
\% \\
\text { Ca } \\
\text { uca } \\
\text { sia } \\
\text { ns }\end{array}$ & $\begin{array}{l}\mathrm{M} / \\
\mathrm{F}: \\
43 \\
/ 5 \\
7\end{array}$ & $\begin{array}{c}<40- \\
>90 \\
\text { year } \\
\mathrm{s}\end{array}$ & $\begin{array}{c}\text { No CI } \\
\text { based } \\
\text { on a } \\
\text { NRPSY } \\
\text { Batter } \\
y \\
\text { descri } \\
\text { ption } \\
\text { report } \\
\text { ed } \\
\text { [Weint } \\
\text { raub } \\
2009]\end{array}$ & Yes & No & $\begin{array}{c}\text { Su } \\
\text { bsa } \\
\text { mp } \\
\text { le }\end{array}$ & No & No & $\begin{array}{c}\mathrm{Su} \\
\mathrm{bsa} \\
\mathrm{mp} \\
\mathrm{le}\end{array}$ & $\begin{array}{c}\text { postm } \\
\text { ortem } \\
\text { neuro } \\
\text { patho } \\
\text { logic } \\
\text { evalu } \\
\text { ations } \\
\text { in a } \\
\text { subsa } \\
\text { mle }\end{array}$ & $\begin{array}{l}\text { https:/ } \\
\text { /www. } \\
\text { alz.wa } \\
\text { shingt } \\
\text { on.edu }\end{array}$ \\
\hline $\begin{array}{l}\text { Nun } \\
\text { Study }\end{array}$ & $\begin{array}{c}67 \\
8\end{array}$ & C & $\begin{array}{l}\mathrm{M} \\
\mathrm{o} \\
\mathrm{n} \\
\mathrm{o}\end{array}$ & $\begin{array}{l}\text { USA, } \\
\text { MN }\end{array}$ & $\begin{array}{l}\text { Eth } \\
\text { nici } \\
\text { ty } \\
\text { not } \\
\text { me } \\
\text { nti } \\
\text { on } \\
\text { ed. } \\
\text { Spe } \\
\text { cifi } \\
\text { city } \\
\text { of } \\
\text { the } \\
\text { coh } \\
\text { ort } \\
\text { po } \\
\text { pul } \\
\text { ati } \\
\text { on: } \\
\text { Nu } \\
\text { ns }\end{array}$ & $\begin{array}{c}\mathrm{M} / \\
\mathrm{F}: \\
0 / \\
10 \\
0\end{array}$ & $\begin{array}{c}\text { Mea } \\
\mathrm{n} 85 \\
\text { year } \\
\mathrm{s}\end{array}$ & $\begin{array}{c}\text { NRPSY } \\
\text { battery } \\
\text { (Delay } \\
\text { ed } \\
\text { Word } \\
\text { Recall, } \\
\text { Word } \\
\text { Recog } \\
\text { nition; } \\
\text { Word } \\
\text { List } \\
\text { Memor } \\
\text { y; } \\
\text { Verbal } \\
\text { Fluenc } \\
\text { y; } \\
\text { Constr } \\
\text { uction } \\
\text { al } \\
\text { Praxis; } \\
\text { Boston } \\
\text { Namin } \\
\text { g; } \\
\text { MMSE) }\end{array}$ & Yes & No & No & No & No & Yes & $\begin{array}{c}\text { postm } \\
\text { ortem } \\
\text { neuro } \\
\text { patho } \\
\text { logic } \\
\text { evalu } \\
\text { ations }\end{array}$ & $\begin{array}{c}\text { Snowd } \\
\text { on et } \\
\text { al. } \\
1996\end{array}$ \\
\hline $\begin{array}{c}\text { SIGN } \\
\text { AL }\end{array}$ & $\begin{array}{c}26 \\
6\end{array}$ & $\mathrm{R}$ & $\begin{array}{c}\mathrm{M} \\
\mathrm{u} \\
\mathrm{lt} \\
\mathrm{i}\end{array}$ & $\begin{array}{c}\text { Spai } \\
\mathrm{n}\end{array}$ & $\begin{array}{c}\text { Not } \\
\text { me } \\
\text { nti } \\
\text { on } \\
\text { ne } \\
\text { d }\end{array}$ & - & $\begin{array}{l}50- \\
75\end{array}$ & $\begin{array}{c}\text { MMSE } \\
\text { score } \\
\geq 24 \\
\text { and } \\
\text { normal } \\
\text { memor } \\
\text { y } \\
\text { perfor } \\
\text { mance } \\
\text { on } \\
\text { FCSRT } \\
\text { Signifi } \\
\text { cant } \\
\text { impair } \\
\text { ment } \\
\text { in } \\
\text { other } \\
\text { cogniti } \\
\text { ve } \\
\text { domai } \\
\text { ns } \\
\text { exclud } \\
\text { ed } \\
\text { throug } \\
\text { h a } \\
\text { formal } \\
\text { cogniti } \\
\text { ve } \\
\text { evalua } \\
\text { tion. }\end{array}$ & Yes & Yes & Yes & No & $\begin{array}{l}\text { Opti } \\
\text { onal }\end{array}$ & Yes & None & $\begin{array}{c}\text { Alcole } \\
\text { a et al } \\
2015\end{array}$ \\
\hline
\end{tabular}




\begin{tabular}{|c|c|c|c|c|c|c|c|c|c|c|c|c|c|c|c|c|}
\hline $\begin{array}{c}\text { WU- } \\
\text { ADRC } \\
\text { (Was } \\
\text { hingt } \\
\text { on } \\
\text { Unive } \\
\text { rsity' } \\
\text { s } \\
\text { Alzhe } \\
\text { imer' } \\
\text { s } \\
\text { Disea } \\
\text { se } \\
\text { Resea } \\
\text { rch } \\
\text { Cente } \\
\text { r } \\
\text { study } \\
\text { ) }\end{array}$ & $\begin{array}{c}34 \\
0\end{array}$ & $\mathrm{R}$ & $\begin{array}{l}\mathrm{M} \\
\mathrm{o} \\
\mathrm{n} \\
\mathrm{o}\end{array}$ & $\begin{array}{l}\text { USA, } \\
\text { MS. }\end{array}$ & $\begin{array}{c}92 \\
\% \\
\mathrm{Ca} \\
\text { uca } \\
\text { sia } \\
\text { ns }\end{array}$ & $\begin{array}{c}\text { M/ } \\
\text { F: } \\
45 \\
/ 5 \\
5\end{array}$ & $\geq 65$ & $\mathrm{CDR}=0$ & Yes & Yes & No & No & No & Yes & No & $\begin{array}{l}\text { Vos SJ } \\
\text { et al., } \\
2013\end{array}$ \\
\hline $\begin{array}{c}\text { WRA } \\
\text { P } \\
\text { (Wisc } \\
\text { onsin } \\
\text { Regis } \\
\text { try } \\
\text { for } \\
\text { Alzhe } \\
\text { imer' } \\
\text { s } \\
\text { Preve } \\
\text { ntion } \\
\text { ) }\end{array}$ & $\begin{array}{c}18 \\
4\end{array}$ & $\mathrm{R}$ & $\begin{array}{l}\mathrm{M} \\
\mathrm{o} \\
\mathrm{n} \\
\mathrm{o}\end{array}$ & $\begin{array}{l}\text { USA, } \\
\text { WI. }\end{array}$ & $\begin{array}{l}98 \\
\% \\
\text { Ca } \\
\text { uca } \\
\text { sia } \\
\text { ns }\end{array}$ & $\begin{array}{l}\mathrm{M} / \\
\mathrm{F}: \\
29 \\
/ 7 \\
1\end{array}$ & $\begin{array}{c}40- \\
65 \\
\text { year } \\
s\end{array}$ & $\begin{array}{l}\text { NRPSY } \\
\text { battery } \\
\text { (Sager } \\
2005 \text { ) }\end{array}$ & Yes & No & No & No & No & Yes & No & $\begin{array}{l}\text { La Rue } \\
\text { A et } \\
\text { al., } \\
2008 \text {; } \\
\text { Sager } \\
\text { MA } \\
2005\end{array}$ \\
\hline
\end{tabular}

$\mathrm{R}=$ Research; $\mathrm{C}=$ Clinical; Mono= Monocentric; Multi= Multicentric; $\mathrm{CI}=$ Cognitive Impairment; GDS=Geriatric Depression Scale; CDR=Clinical Dementia Rating Scale; FCSRT: Free and Cued Selective Reminding Test; NRPSY=Neuropsychological; MMSE Mini Mental State Examination. NA=Not Applicable

For some monocentric studies the name of center is reported as some cohorts may be pooled in the publication.

1 - Weiner MW(1), Aisen PS, Jack CR Jr, Jagust WJ, Trojanowski JQ, Shaw L, Saykin AJ, Morris JC, Cairns N, Beckett LA, Toga A, Green R, Walter S, Soares H, Snyder P,Siemers E, Potter W, Cole PE, Schmidt M; Alzheimer's Disease Neuroimaging Initiative.The Alzheimer's disease neuroimaging initiative: progress report and future plans.. Alzheimers Dement. 2010 May;6(3):202-11.e7. doi: 10.1016/j.jalz.2010.03.007.

2 - van der Flier WM, Pijnenburg YA, Prins N, Lemstra AW, Bouwman FH, Teunissen CE, van Berckel BN, Stam CJ, Barkhof F, Visser PJ, van Egmond E, Scheltens P. Optimizing patient care and research: the Amsterdam Dementia Cohort. J Alzheimers Dis. 2014;41(1):313-27.

3 - Ellis KA, Bush AI, Darby D, De Fazio D, Foster J, Hudson P, Lautenschlager NT, Lenzo N, Martins RN, Maruff P, Masters C, Milner A, Pike K, Rowe C, Savage G, Szoeke C, Taddei K, Villemagne V, Woodward M, Ames D; AIBL Research Group. The Australian Imaging, Biomarkers and Lifestyle (AIBL) study of aging: methodology and baseline characteristics of 1112 individuals recruited for a longitudinal study of Alzheimer's disease. Int Psychogeriatr. 2009 Aug;21(4):672-87. 
4 - Greenwood PM, Lambert C, Sunderland T, Parasuraman R.4Effects of apolipoprotein E genotype on spatial attention, working memory, and their interaction in healthy, middle-aged adults: results From the National Institute of Mental Health's BIOCARD study.

Neuropsychology. 2005 Mar;19(2):199-211.

5 - Palmqvist S, Zetterberg H, Blennow K(2), Vestberg S, Andreasson U, Brooks DJ, Owenius R, Hägerström D, Wollmer P, Minthon L, Hansson O.Accuracy of brain amyloid detection in clinical practice using cerebrospinal fluid $\beta$-amyloid 42: a cross-validation study against amyloid positron emission tomography. JAMA Neurol. 2014 Oct;71(10):1282-9. doi: 10.1001/jamaneurol.2014.1358.

6 - The Baltimore Longitudinal Study on Aging (BLSA):

https://clinicaltrials.gov/ct2/show/NCT00233272

7 - Dagley A, LaPoint M, Huijbers W, Hedden T, McLaren DG, Chatwal JP, Papp KV, Amariglio RE, Blacker D, Rentz DM, Johnson KA, Sperling RA, Schultz AP. Harvard Aging Brain Study: Dataset and accessibility. Neuroimage. 2015 Apr 3. pii: S1053-8119(15)00265-7.

8 - Roberts RO, Geda YE, Knopman DS, Cha RH, Pankratz VS, Boeve BF, Ivnik RJ, Tangalos EG, Petersen RC, Rocca WA. The Mayo Clinic Study of Aging: design and sampling, participation, baseline measures and sample characteristics. Neuroepidemiology. 2008;30(1):58-69.

Roberts RO, Cha RH, Knopman DS, Petersen RC, Rocca WA. Postmenopausal estrogen therapy and Alzheimer disease: overall negative findings. Alzheimer Dis Assoc Disord. 2006 Jul-Sep;20(3):141-6.

9 - National Alzheimer's Coordinating Center (NACC)

https://www.alz.washington.edu/cgibin/broker93?_service=naccnew9\&_program=naccwww. pubrep1.sas\&TYPEF=DISPLAYIDS

10 - Snowdon DA, Kemper SJ, Mortimer JA, Greiner LH, Wekstein DR, Markesbery WR. Linguistic ability in early life and cognitive function and Alzheimer's disease in late life. Findings from the Nun Study.JAMA. 1996 Feb 21;275(7):528-32.

\section{1- SIGNAL: http://signalstudy.es/en/objectives.html}

Alcolea D, Martínez-Lage P, Sánchez-Juan P, Olazarán J, Antúnez C, Izagirre A, Ecay-Torres M, Estanga A, Clerigué M, Guisasola MC,

Sánchez Ruiz D, Marín Muñoz J, Calero M, Blesa R, Clarimón J, Carmona-Iragui M, Morenas-Rodríguez E, Rodríguez-Rodríguez E, Vázquez

Higuera JL, Fortea J, Lleó A. Amyloid precursor protein metabolism and inflammation markers in preclinical

Alzheimer disease. Neurology. 2015 Aug 18;85(7):626-33. doi:

10.1212/WNL.0000000000001859. Epub 2015

Jul 15. 
12- Vos SJ, Xiong C, Visser PJ, Jasielec MS, Hassenstab J, Grant EA, Cairns NJ, Morris JC, Holtzman DM, Fagan AM. Preclinical Alzheimer's disease and its outcome: a longitudinal cohort study. Lancet Neurol. 2013 Oct;12(10):957-65.

13- Sager MA, Hermann B, La Rue A. Middle-aged children of persons with Alzheimer's disease: APOE genotypes and cognitive function in the Wisconsin Registry for Alzheimer's Prevention. J Geriatr Psychiatry Neurol. 2005 Dec;18(4):245-9.

La Rue A, Hermann B, Jones JE, Johnson S, Asthana S, Sager MA. Effect of parental family history of Alzheimer's disease on serial position profiles. Alzheimers Dement. 2008 Jul;4(4):285-90. doi: 10.1016/j.jalz.2008.03.009. 

Cohort(s) used

for the study

[or center for

unnamed

monocentric

studies)]

Total ge $\mathbf{N}(\%$

total

populatio

n)

\begin{tabular}{|c|c|}
\hline N PC & stage \\
N $(\%$ & 1 \\
total & N (\% \\
popula & of \\
tion) & PC)
\end{tabular}

stage

stag N AD

\begin{tabular}{|l|l|l|}
$\mathbf{N}$ & $\mathbf{N}$ & $\mathbf{N}$ other \\
\hline
\end{tabular}

\begin{tabular}{|c|c} 
N other & ApoE \\
N $(\%$ of & $4+$
\end{tabular}

$\mathrm{N}(\%$

N

$\mathrm{N}$
$(\%$

of

or

MCI

total

in

PC)

PC)

popu

PART

of

populati total

total

on)

popul

ation

n) total ation)

(\%)

CROSS-SECTIONAL STUDIES

\begin{tabular}{|c|c|c|c|c|c|c|c|c|c|c|c|c|c|}
\hline $\begin{array}{l}\text { (Morris, Roe } \\
\text { et al. 2010) }\end{array}$ & WU-ADRC & 241 & $\begin{array}{l}66 \\
, 8 \\
\end{array}$ & - & $44(18)$ & - & - & - & - & - & - & - & $\begin{array}{c}82 \\
(34) \\
\end{array}$ \\
\hline $\begin{array}{l}\text { (Pike, Ellis et } \\
\text { al. 2011) }\end{array}$ & AIBL & 177 & 70 & $\begin{array}{l}119 \\
(67)\end{array}$ & $\begin{array}{c}58 \\
(33)\end{array}$ & - & - & - & - & - & - & - & - \\
\hline $\begin{array}{c}\text { (Mielke, } \\
\text { Wiste et al. } \\
\text { 2012) }\end{array}$ & MCSA & 483 & - & $\begin{array}{l}332 \\
(69)\end{array}$ & $\begin{array}{l}151 \\
(31)\end{array}$ & - & - & - & - & - & - & - & $\begin{array}{l}121 \\
(25)\end{array}$ \\
\hline $\begin{array}{c}\text { (Amariglio, } \\
\text { Becker et al. } \\
2012)\end{array}$ & HABS & 131 & $\begin{array}{l}73 \\
, 5\end{array}$ & $\begin{array}{l}97 \\
(74)\end{array}$ & $\begin{array}{c}34 \\
(26)\end{array}$ & - & - & - & - & - & - & - & - \\
\hline $\begin{array}{c}\text { (Jack, } \\
\text { Knopman et } \\
\text { al. 2012) } \\
\end{array}$ & MCSA & 450 & 78 & $\begin{array}{l}193 \\
(43)\end{array}$ & $\begin{array}{l}139 \\
(31)\end{array}$ & $\begin{array}{c}70 \\
(50)\end{array}$ & $\begin{array}{c}56 \\
(40)\end{array}$ & $\begin{array}{l}13 \\
(9)\end{array}$ & $\begin{array}{l}42 \\
(9)\end{array}$ & $\begin{array}{l}103 \\
(23)\end{array}$ & - & $\begin{array}{l}15 \\
(3)\end{array}$ & $\begin{array}{l}117 \\
(26)\end{array}$ \\
\hline $\begin{array}{l}\text { (Harrington, } \\
\text { Chiang et al. } \\
\text { 2013) } \\
\end{array}$ & [Pasadena (Cal)] & 149 & 78 & $\begin{array}{l}36 \\
(24)\end{array}$ & $\begin{array}{c}34 \\
(23)\end{array}$ & - & - & - & $\begin{array}{l}29 \\
(19)\end{array}$ & - & $\begin{array}{c}40 \\
(27 \\
) \\
\end{array}$ & $\begin{array}{l}10 \\
(7)\end{array}$ & - \\
\hline $\begin{array}{l}\text { (Whitwell, } \\
\text { Tosakulwong } \\
\text { et al. 2013) }\end{array}$ & $\begin{array}{c}\text { Mayo Clinic } \\
\text { ADRC cohort or } \\
\text { MCSA }\end{array}$ & 318 & 80 & $\begin{array}{l}115 \\
(36)\end{array}$ & $\begin{array}{l}115 \\
(36)\end{array}$ & - & - & - & - & - & $\begin{array}{l}88 \\
(28 \\
)\end{array}$ & - & - \\
\hline $\begin{array}{c}(\mathrm{Ju}, \\
\text { McLeland et } \\
\text { al. 2013) }\end{array}$ & WU-ADRC & 142 & $\begin{array}{r}65 \\
, 6\end{array}$ & $\begin{array}{l}110 \\
(77)\end{array}$ & $\begin{array}{c}32 \\
(23)\end{array}$ & - & - & - & - & - & - & - & $\begin{array}{c}52 \\
(36.6)\end{array}$ \\
\hline $\begin{array}{c}\text { (Knopman, } \\
\text { Jack et al. } \\
\text { 2013) }\end{array}$ & MCSA & 430 & 78 & $\begin{array}{l}191 \\
(44)\end{array}$ & $\begin{array}{l}137 \\
(32)\end{array}$ & $\begin{array}{c}68 \\
(50)\end{array}$ & $\begin{array}{c}56 \\
(41)\end{array}$ & $\begin{array}{l}13 \\
(9)\end{array}$ & - & $\begin{array}{l}102 \\
(24)\end{array}$ & - & - & $\begin{array}{l}107 \\
(25)\end{array}$ \\
\hline $\begin{array}{l}\text { (Brier, } \\
\text { Thomas et al. } \\
\text { 2014) }\end{array}$ & WU-ADRC & 297 & 68 & $\begin{array}{l}200 \\
(67)\end{array}$ & $\begin{array}{c}97 \\
(33)\end{array}$ & - & - & - & - & - & - & - & - \\
\hline $\begin{array}{l}\text { (Brier, } \\
\text { Thomas et al. } \\
\text { 2014) }\end{array}$ & WU-ADRC & 326 & 69 & $\begin{array}{l}132 \\
(40)\end{array}$ & $\begin{array}{l}59 \\
(18)\end{array}$ & $\begin{array}{c}46 \\
(78)\end{array}$ & $\begin{array}{l}13 \\
(22)\end{array}$ & - & $\begin{array}{l}31 \\
(9)\end{array}$ & - & $\begin{array}{c}90 \\
(28 \\
) \\
\end{array}$ & $\begin{array}{l}14 \\
(4)\end{array}$ & - \\
\hline $\begin{array}{l}\text { (Jack, Wiste } \\
\text { et al. 2014) }\end{array}$ & MCSA & 985 & 74 & $\begin{array}{l}503 \\
(51)\end{array}$ & $\begin{array}{l}352 \\
(36)\end{array}$ & $\begin{array}{l}213 \\
(60)\end{array}$ & $\begin{array}{l}130 \\
(40)\end{array}$ & - & - & $\begin{array}{l}139 \\
(14)\end{array}$ & - & - & $\begin{array}{l}256 \\
(26)\end{array}$ \\
\hline $\begin{array}{c}\text { (Racine, } \\
\text { Adluru et al. } \\
\text { 2014) }\end{array}$ & WRAP & 139 & $\begin{array}{c}60 \\
, 6\end{array}$ & $\begin{array}{l}112 \\
(81)\end{array}$ & $\begin{array}{c}27 \\
(19)\end{array}$ & - & - & - & - & - & - & - & - \\
\hline $\begin{array}{l}\text { (Fortea, } \\
\text { Vilaplana et } \\
\text { al. 2014) } \\
\end{array}$ & ADNI & 145 & $\begin{array}{l}73 \\
, 4\end{array}$ & $\begin{array}{c}74 \\
(51)\end{array}$ & $\begin{array}{c}39 \\
(27)\end{array}$ & $\begin{array}{c}8 \\
(21)\end{array}$ & $\begin{array}{c}31 \\
(79)\end{array}$ & - & - & $\begin{array}{c}32 \\
(22)\end{array}$ & - & - & - \\
\hline $\begin{array}{l}\text { (Wang, } \\
\text { Benzinger et } \\
\text { al. 2015) } \\
\end{array}$ & WU-ADRC & 188 & 73 & - & - & - & - & - & - & - & - & - & - \\
\hline $\begin{array}{c}\text { (Doherty, } \\
\text { Schultz et al. } \\
\text { 2015) }\end{array}$ & WRAP & 109 & $\begin{array}{c}60 \\
, 7\end{array}$ & $74(68)$ & $35(32)$ & - & - & - & - & - & - & - & $\begin{array}{l}45 \\
(41)\end{array}$ \\
\hline $\begin{array}{l}\text { (Valech, } \\
\text { Mollica et al. } \\
\text { 2015) }\end{array}$ & [Barcelona] & 111 & & $\begin{array}{c}59 \\
(53)\end{array}$ & $19(24)$ & - & - & - & $10(9)$ & - & $\begin{array}{l}23 \\
(21 \\
)\end{array}$ & - & - \\
\hline
\end{tabular}




\begin{tabular}{c|c|c|c|c|c|c|c|c|c|c|c|c|c}
\hline $\begin{array}{c}\text { (Jack, Wiste } \\
\text { et al. 2015) }\end{array}$ & MCSA & 1331 & 71 & - & - & - & - & - & - & - & - & - & 27 \\
$\begin{array}{c}\text { (Alcolea, } \\
\text { Martinez- } \\
\text { Lage et al. } \\
\text { 2015) }\end{array}$ & SIGNAL & 266 & 58 & $203(76.3)$ & $\begin{array}{c}36 \\
(13.5)\end{array}$ & $\begin{array}{c}26 \\
(72)\end{array}$ & $\begin{array}{c}<10 \\
(28)\end{array}$ & $\begin{array}{c}<10 \\
(28)\end{array}$ & 0 & $\begin{array}{c}27 \\
(10.1)\end{array}$ & 0 & 0 & 67 \\
\hline $\begin{array}{c}\text { (Papp, } \\
\text { Amariglio et } \\
\text { al. 2015) }\end{array}$ & HABS & 260 & 73 & $126(48)$ & $70(27)$ & $\begin{array}{c}32 \\
(46)\end{array}$ & $\begin{array}{c}38 \\
(54)\end{array}$ & - & - & $64(25)$ & - & - & - \\
\hline $\begin{array}{c}\text { (Hassenstab, } \\
\text { Chasse et al. } \\
\text { 2016) }\end{array}$ & WU-ADRC & 264 & 72 & $177(67)$ & $87(33)$ & - & - & - & - & - & - & - & 83 \\
\hline $\begin{array}{c}\text { Voevodskaya } \\
\text { Sundgren et } \\
\text { al. 2016) }\end{array}$ & BioFINDER & 352 & 72 & $156(44)$ & $\begin{array}{c}108 \\
(31)\end{array}$ & $\begin{array}{c}59 \\
(55)\end{array}$ & - & $\begin{array}{c}49 \\
(45)\end{array}$ & - & - & $\begin{array}{c}88 \\
(25\end{array}$ & - & $\begin{array}{c}142 \\
(40)\end{array}$ \\
\hline
\end{tabular}

\section{LONGITUDINAL STUDIES}

\begin{tabular}{|c|c|c|c|c|c|c|c|c|c|c|c|c|c|}
\hline $\begin{array}{l}\text { (Morris, Roe } \\
\text { et al. 2009) }\end{array}$ & WU-ADRC & 159 & $\begin{array}{r}71 \\
, 5 \\
\end{array}$ & - & - & - & - & - & - & - & - & - & - \\
\hline $\begin{array}{c}\text { (Craig- } \\
\text { Schapiro, } \\
\text { Perrin et al. } \\
\text { 2010) }\end{array}$ & WU-ADRC & 340 & 71 & - & - & - & - & - & - & - & - & - & - \\
\hline $\begin{array}{c}\text { (Desikan, } \\
\text { McEvoy et al. } \\
\text { 2012) }\end{array}$ & ADNI & 115 & 76 & $\begin{array}{c}46 \\
(40)\end{array}$ & $\begin{array}{c}41 \\
(36)\end{array}$ & $\begin{array}{c}20 \\
(49)\end{array}$ & $\begin{array}{c}21 \\
(51)\end{array}$ & - & - & $\begin{array}{c}19 \\
(17)\end{array}$ & - & - & $\begin{array}{c}27 \\
(23.3)\end{array}$ \\
\hline $\begin{array}{c}\text { (Knopman, } \\
\text { Jack et al. } \\
\text { 2012) }\end{array}$ & MCSA & 286 & 79 & $\begin{array}{l}127 \\
(44)\end{array}$ & $\begin{array}{c}90 \\
(31)\end{array}$ & $\begin{array}{c}41 \\
(46)\end{array}$ & $\begin{array}{c}39 \\
(43)\end{array}$ & $\begin{array}{c}7 \\
(8)\end{array}$ & - & $\begin{array}{c}69 \\
(24)\end{array}$ & - & - & $\begin{array}{c}74 \\
(26)\end{array}$ \\
\hline $\begin{array}{l}\text { (van Harten, } \\
\text { Smits et al. } \\
\text { 2013) }\end{array}$ & $\begin{array}{c}\text { Amsterdam } \\
\text { dementia cohort }\end{array}$ & 132 & 61 & $\begin{array}{c}80 \\
(60)\end{array}$ & $\begin{array}{c}21 \\
(16)\end{array}$ & $\begin{array}{c}11 \\
(52)\end{array}$ & $\begin{array}{c}10 \\
(48)\end{array}$ & - & - & $\begin{array}{c}31 \\
(23)\end{array}$ & - & - & $\begin{array}{c}54 \\
(41)\end{array}$ \\
\hline $\begin{array}{l}\text { (Vos, Xiong } \\
\text { et al. 2013) }\end{array}$ & WU-ADRC & 311 & $\begin{array}{r}72 \\
.9 \\
\end{array}$ & $\begin{array}{l}129 \\
(41) \\
\end{array}$ & $\begin{array}{c}96 \\
(31) \\
\end{array}$ & $\begin{array}{c}47 \\
(49) \\
\end{array}$ & $\begin{array}{c}36 \\
(38) \\
\end{array}$ & $\begin{array}{c}13 \\
(14) \\
\end{array}$ & $\begin{array}{c}0 \\
(0) \\
\end{array}$ & $\begin{array}{c}72 \\
(23) \\
\end{array}$ & - & $\begin{array}{l}14 \\
(5) \\
\end{array}$ & $\begin{array}{l}106 \\
(34) \\
\end{array}$ \\
\hline $\begin{array}{l}\text { (Stark, Roe et } \\
\text { al. 2013) }\end{array}$ & WU-ADRC & 119 & $\begin{array}{l}74 \\
.4 \\
\end{array}$ & $\begin{array}{l}101 \\
(85) \\
\end{array}$ & $\begin{array}{c}18 \\
(15) \\
\end{array}$ & - & - & - & - & - & - & - & - \\
\hline $\begin{array}{c}\text { (Lim, } \\
\text { Villemagne et } \\
\text { al. 2013) }\end{array}$ & AIBL & 165 & $\begin{array}{l}71 \\
4\end{array}$ & $\begin{array}{l}116 \\
(70)\end{array}$ & $\begin{array}{c}49 \\
(30)\end{array}$ & - & - & - & - & - & - & - & $\begin{array}{c}70 \\
(42,4)\end{array}$ \\
\hline $\begin{array}{l}\text { (Lim, Maruff } \\
\text { et al. 2014) }\end{array}$ & AIBL & 333 & 70 & $\begin{array}{l}249 \\
(75)\end{array}$ & $\begin{array}{c}84 \\
(25) \\
\end{array}$ & - & - & - & - & - & - & - & $\begin{array}{c}109 \\
(32,7)\end{array}$ \\
\hline $\begin{array}{l}\text { (Mormino, } \\
\text { Betensky et } \\
\text { al. 2014) }\end{array}$ & HABS & 166 & 74 & $\begin{array}{c}81 \\
(49)\end{array}$ & $\begin{array}{c}47 \\
(28)\end{array}$ & $\begin{array}{c}19 \\
(40)\end{array}$ & $\begin{array}{c}28 \\
(60)\end{array}$ & - & - & $\begin{array}{l}38 \\
(23)\end{array}$ & - & - & $\begin{array}{c}50 \\
(30)\end{array}$ \\
\hline $\begin{array}{l}\text { (Donohue, } \\
\text { Sperling et al. } \\
\text { 2014) }\end{array}$ & ADNI/AIBL & $\begin{array}{c}97 / 16 \\
4\end{array}$ & $\begin{array}{c}75 \\
, 8 \\
1 / \\
71 \\
, 3 \\
7\end{array}$ & $\begin{array}{c}60 / 114(62 / \\
70)\end{array}$ & $\begin{array}{c}37 / 50 \\
(38 / 30)\end{array}$ & - & - & - & - & - & - & - & $\begin{array}{c}24 / 70 \\
(25 / 4 \\
3)\end{array}$ \\
\hline $\begin{array}{l}\text { (Ayutyanont, } \\
\text { Langbaum et } \\
\text { al. 2014) }\end{array}$ & $\begin{array}{l}\text { E280A } \\
\text { Antioquia } \\
\text { cohort }\end{array}$ & 134 & 44 & $\begin{array}{c}78 \\
(58)\end{array}$ & $\begin{array}{c}56 \\
(42)\end{array}$ & - & - & - & - & - & - & - & - \\
\hline $\begin{array}{l}\text { (Mormino, } \\
\text { Betensky et } \\
\text { al. 2014) }\end{array}$ & $\begin{array}{c}\text { HABS/ADNI/A } \\
\text { IBL }\end{array}$ & 490 & 75 & $\begin{array}{l}355 \\
(72)\end{array}$ & $\begin{array}{l}135 \\
(28)\end{array}$ & - & - & - & - & - & - & - & $\begin{array}{l}225 \\
(46)\end{array}$ \\
\hline
\end{tabular}




\begin{tabular}{|c|c|c|c|c|c|c|c|c|c|c|c|c|c|}
\hline $\begin{array}{l}\text { (Pietrzak, Lim } \\
\text { et al. 2015) }\end{array}$ & AIBL & 333 & 70 & $\begin{array}{l}249 \\
(75)\end{array}$ & $\begin{array}{c}84 \\
(25)\end{array}$ & - & - & - & - & - & - & - & $\begin{array}{c}109 \\
(32,7)\end{array}$ \\
\hline $\begin{array}{l}\text { (Pietrzak, Lim } \\
\text { et al. 2015) }\end{array}$ & AIBL & 333 & 70 & $\begin{array}{l}249 \\
(75)\end{array}$ & $\begin{array}{c}84 \\
(25)\end{array}$ & - & - & - & - & - & - & - & $\begin{array}{c}109 \\
(32,7)\end{array}$ \\
\hline $\begin{array}{l}\text { (Nation, } \\
\text { Edmonds et } \\
\text { al. 2015) }\end{array}$ & ADNI & 877 & $\begin{array}{l}72 \\
, 6\end{array}$ & - & - & - & - & - & - & $\begin{array}{l}167 \\
(19)\end{array}$ & $\begin{array}{l}602 \\
(69)\end{array}$ & - & $\begin{array}{l}360 \\
(41)\end{array}$ \\
\hline $\begin{array}{l}\text { (Thai, Lim et } \\
\text { al. 2015) }\end{array}$ & AIBL & 317 & $\begin{array}{r}69 \\
.9\end{array}$ & - & $76(24)$ & - & - & - & - & - & - & - & $\begin{array}{l}104 \\
(33)\end{array}$ \\
\hline $\begin{array}{c}\text { (Pettigrew, } \\
\text { Soldan et al. } \\
2015)\end{array}$ & BIOCARD & 302 & $\begin{array}{c}56 \\
.6\end{array}$ & $240(79)$ & $62(21)$ & - & - & - & - & - & - & - & $\begin{array}{c}94 \\
(32)\end{array}$ \\
\hline $\begin{array}{c}\text { (Sutphen, } \\
\text { Jasielec et al. } \\
\text { 2015) }\end{array}$ & WU-ADRC & 169 & $\begin{array}{r}60 \\
.7\end{array}$ & $118(70)$ & $51(30)$ & - & - & - & - & - & - & - & $\begin{array}{c}61(36 \\
)\end{array}$ \\
\hline $\begin{array}{c}\text { (Papp, } \\
\text { Mormino et } \\
\text { al. 2016) }\end{array}$ & HABS & 275 & & $205(75)$ & $70(25)$ & - & - & - & - & - & - & - & - \\
\hline $\begin{array}{l}\text { (Soldan, } \\
\text { Pettigrew et } \\
\text { al. 2016) }\end{array}$ & BIOCARD & 222 & $\begin{array}{c}56 \\
.9\end{array}$ & $102(46)$ & $74(33)$ & $\begin{array}{c}46 \\
(62)\end{array}$ & $\begin{array}{c}28 \\
(38)\end{array}$ & - & - & $46(21)$ & - & - & $\begin{array}{c}73 \\
(33)\end{array}$ \\
\hline $\begin{array}{c}\text { (Racine, } \\
\text { Koscik et al. } \\
2016)\end{array}$ & $\begin{array}{l}\text { WRAP and } \\
\text { Wisconsin } \\
\text { ADRC }\end{array}$ & 175 & 59 & $76(43)$ & $54(31)$ & - & - & - & - & $45(26)$ & - & - & $\begin{array}{c}79 \\
(45)\end{array}$ \\
\hline $\begin{array}{c}\text { (Pascoal, } \\
\text { Mathotaarach } \\
\text { chi et al. } \\
\text { 2016) }\end{array}$ & ADNI & 120 & $\begin{array}{c}74 \\
.9\end{array}$ & - & - & - & - & - & - & - & - & - & - \\
\hline $\begin{array}{l}\text { (Vos, Gordon } \\
\text { et al. 2016)i }\end{array}$ & WU-ADRC & 212 & $\begin{array}{l}66 \\
.1\end{array}$ & $\begin{array}{l}\text { imaging: } \\
\text { 127 (60), } \\
\text { CSF: } 114 \\
(54)\end{array}$ & $\begin{array}{c}\text { imagin } \\
\text { g: } 45 \\
(21), \\
\text { CSF:58 } \\
(27)\end{array}$ & $\begin{array}{c}\text { imagi } \\
\text { ng: } \\
26, \\
\text { CSF:4 } \\
2 \\
\end{array}$ & $\begin{array}{c}\text { imag } \\
\text { ing: } \\
19 \text {, } \\
\text { CSF: } \\
16 \\
\end{array}$ & - & - & 40 (19) & - & - & $\begin{array}{c}70 \\
(33)\end{array}$ \\
\hline $\begin{array}{c}\text { (Bilgel, } \\
\text { Prince et al. } \\
\text { 2016) }\end{array}$ & BLSA & 104 & 77 & - & - & - & - & - & - & - & - & - & - \\
\hline $\begin{array}{c}\text { (Brier, } \\
\text { McCarthy et } \\
\text { al. 2016) }\end{array}$ & WU-ADRC & 157 & $\begin{array}{l}61 \\
.6\end{array}$ & $131(83)$ & $26(17)$ & - & - & - & - & - & - & - & $\begin{array}{c}49 \\
(31.2)\end{array}$ \\
\hline $\begin{array}{c}\text { (Clark, } \\
\text { Racine et al. } \\
2016)\end{array}$ & WRAP & 184 & $\begin{array}{l}58 \\
.6\end{array}$ & $156(84.8)$ & $\begin{array}{c}28 \\
(15.2)\end{array}$ & - & - & - & - & - & - & - & $\begin{array}{c}73 \\
(40)\end{array}$ \\
\hline $\begin{array}{l}\text { (Harrington, } \\
\text { Gould et al. } \\
2016)\end{array}$ & AIBL & 359 & $\begin{array}{l}69 \\
.7\end{array}$ & $278(77)$ & $\begin{array}{c}81 \\
(22.6)\end{array}$ & - & - & - & - & - & - & - & - \\
\hline $\begin{array}{l}\text { (Lim, Snyder } \\
\text { et al. 2016) }\end{array}$ & AIBL & 423 & $\begin{array}{r}69 \\
, 4\end{array}$ & $326(77)$ & $\begin{array}{c}97 \\
(23)\end{array}$ & - & - & - & - & - & - & - & $\begin{array}{l}115 \\
(27)\end{array}$ \\
\hline
\end{tabular}

NEUROPATHOLOGICAL STUDIES

\begin{tabular}{c|c|c|c|c|c|c|c|c|c|c|c|c|c|c|}
\hline $\begin{array}{c}\text { (Jicha, Abner } \\
\text { et al. 2012) }\end{array}$ & $\begin{array}{c}\text { UK-ADC } \\
\text { (Kentucky) }\end{array}$ & 126 & 85 & 59 & 54 & 13 & 15 & 26 & 14 & 13 & 24 & - & 38 \\
$(10)$ & $(19)$ & $(28)$ & $(48)$ & $(11)$ & $(10)$ \\
\hline $\begin{array}{c}\text { (Abner, } \\
\text { Kryscio et al. } \\
2011)\end{array}$ & $\begin{array}{c}\text { NACC and Nun } \\
\text { Study }\end{array}$ & 2451 & - & - & - & - & - & - & - & - & - & - \\
\hline
\end{tabular}




\begin{tabular}{c|c|c|c|c|c|c|c|c|c|c|c|c|c}
\hline $\begin{array}{c}\text { Riley, Jicha } \\
\text { et al. 2011) }\end{array}$ & $\begin{array}{c}\text { UK-ADC } \\
\text { (Kentucky) }\end{array}$ & 121 & 76 & $\begin{array}{c}89 \\
(74)\end{array}$ & $\begin{array}{c}32 \\
(26)\end{array}$ & - & - & - & - & - & - & - & 34 \\
\hline $\begin{array}{c}\text { Thal, von } \\
\text { Arnim et al. } \\
2013)\end{array}$ & $\begin{array}{c}\text { Multicentric } \\
\text { autopsy study. }\end{array}$ & 766 & 74 & 404 & 248 & - & - & - & 114 & - & - & - & - \\
$(152)$ &, 7 & $(53)$ & & & & & & & & & \\
\end{tabular}

TABLE 1. DESCRIPTION OF STUDIES POPULATIONS

ADNI : Alzheimer's Disease Neuroimaging Initiative ; AIBL: Australian Imaging, Biomarkers and Lifestyle Flagship Study of Ageing; BIOCARD: Biomarkers of Cognitive Decline Among Normal Individuals; HABS: Harvard Aging Brain Study ; MCSA: Mayo Clinic Study of Aging Mayo Clinic ADRC: Mayo Clinic Alzheimer Disease Research Center; NACC : National Alzheimer's Coordinating Center database; SIGNAL study: Spanish project on biomarkers in the preclinical phase of Alzheimer Disease (AD). UK-ADC: University of Kentucky, Alzheimer Disease Center ; BioFINDER: Biomarkers For Identifying Neurodegenerative Disorders Early and Reliably (Sweden); VA SanDiego : Veteran Administration San Diego, CAL ; WU-ADRC : Charles and Joanne Knight Alzheimer's Disease Research Center at Washington University in Saint Louis ; WRAP : Wisconsin Registry for Alzheimer's Prevention. Multicentric autopsy study: All autopsy brains collected from individuals who died in university or municipal Hospitals in Germany (Bonn, Frankfurt/Main, Mainz, Offenbach/Main, Ulm), USA (Little Rock, AR), the United Kingdom (Newcastle upon Tyne), or Austria (Vienna)

HC : Healthy Controls

PC: Preclinical

AD : Alzheimer Disease

MCI : Mild Cognitive Impairment

SNAP : Suspected Non-Amyloid Pathology

PART : Primary Age Related Taupathy

Stage 1-3: according to NIA-AA proposed classification of preclinical $\mathrm{AD}$

Abner, E. L., R. J. Kryscio, F. A. Schmitt, K. S. Santacruz, G. A. Jicha, Y. Lin, J. M. Neltner, C. D. Smith, L. J. Van Eldik and P. T. Nelson (2011). ""End-stage" neurofibrillary tangle pathology in preclinical Alzheimer's disease: fact or fiction?" J Alzheimers Dis 25(3): 445453. 
Alcolea, D., P. Martinez-Lage, P. Sanchez-Juan, J. Olazaran, C. Antunez, A. Izagirre, M. Ecay-Torres, A. Estanga, M. Clerigue, M. C. Guisasola, D. Sanchez Ruiz, J. Marin Munoz, M. Calero, R. Blesa, J. Clarimon, M. Carmona-Iragui, E. Morenas-Rodriguez, E. RodriguezRodriguez, J. L. Vazquez Higuera, J. Fortea and A. Lleo (2015). "Amyloid precursor protein metabolism and inflammation markers in preclinical Alzheimer disease." Neurology 85(7): 626-633.

Amariglio, R. E., J. A. Becker, J. Carmasin, L. P. Wadsworth, N. Lorius, C. Sullivan, J. E. Maye, C. Gidicsin, L. C. Pepin, R. A. Sperling, K. A. Johnson and D. M. Rentz (2012).

"Subjective cognitive complaints and amyloid burden in cognitively normal older individuals." Neuropsychologia 50(12): 2880-2886.

Ayutyanont, N., J. B. Langbaum, S. B. Hendrix, K. Chen, A. S. Fleisher, M. Friesenhahn, M. Ward, C. Aguirre, N. Acosta-Baena, L. Madrigal, C. Munoz, V. Tirado, S. Moreno, P. N. Tariot, F. Lopera and E. M. Reiman (2014). "The Alzheimer's prevention initiative composite cognitive test score: sample size estimates for the evaluation of preclinical Alzheimer's disease treatments in presenilin 1 E280A mutation carriers." J Clin Psychiatry 75(6): 652-660. Bilgel, M., J. L. Prince, D. F. Wong, S. M. Resnick and B. M. Jedynak (2016). "A multivariate nonlinear mixed effects model for longitudinal image analysis: Application to amyloid imaging." Neuroimage 134: 658-670.

Brier, M. R., J. E. McCarthy, T. L. Benzinger, A. Stern, Y. Su, K. A. Friedrichsen, J. C. Morris, B. M. Ances and A. G. Vlassenko (2016). "Local and distributed PiB accumulation associated with development of preclinical Alzheimer's disease." Neurobiol Aging 38: 104111.

Brier, M. R., J. B. Thomas, A. M. Fagan, J. Hassenstab, D. M. Holtzman, T. L. Benzinger, J. C. Morris and B. M. Ances (2014). "Functional connectivity and graph theory in preclinical Alzheimer's disease." Neurobiol Aging 35(4): 757-768.

Brier, M. R., J. B. Thomas, A. Z. Snyder, L. Wang, A. M. Fagan, T. Benzinger, J. C. Morris and B. M. Ances (2014). "Unrecognized preclinical Alzheimer disease confounds rs-fcMRI studies of normal aging." Neurology 83(18): 1613-1619.

Clark, L. R., A. M. Racine, R. L. Koscik, O. C. Okonkwo, C. D. Engelman, C. M. Carlsson, S. Asthana, B. B. Bendlin, R. Chappell, C. R. Nicholas, H. A. Rowley, J. M. Oh, B. P. Hermann, M. A. Sager, B. T. Christian and S. C. Johnson (2016). "Beta-amyloid and cognitive decline in late middle age: Findings from the Wisconsin Registry for Alzheimer's Prevention study." Alzheimers Dement.

Craig-Schapiro, R., R. J. Perrin, C. M. Roe, C. Xiong, D. Carter, N. J. Cairns, M. A. Mintun, E. R. Peskind, G. Li, D. R. Galasko, C. M. Clark, J. F. Quinn, G. D'Angelo, J. P. Malone, R. R. Townsend, J. C. Morris, A. M. Fagan and D. M. Holtzman (2010). "YKL-40: a novel prognostic fluid biomarker for preclinical Alzheimer's disease." Biol Psychiatry 68(10): 903912.

Desikan, R. S., L. K. McEvoy, W. K. Thompson, D. Holland, J. B. Brewer, P. S. Aisen, R. A. Sperling, A. M. Dale and I. Alzheimer's Disease Neuroimaging (2012). "Amyloid-beta-associated clinical decline occurs only in the presence of elevated P-tau." Arch Neurol 69(6): 709-713.

Doherty, B. M., S. A. Schultz, J. M. Oh, R. L. Koscik, N. M. Dowling, T. E. Barnhart, D. Murali, C. L. Gallagher, C. M. Carlsson, B. B. Bendlin, A. LaRue, B. P. Hermann, H. A. Rowley, S. Asthana, M. A. Sager, B. T. Christian, S. C. Johnson and O. C. Okonkwo (2015). "Amyloid burden, cortical thickness, and cognitive function in the Wisconsin Registry for Alzheimer's Prevention." Alzheimers Dement (Amst) 1(2): 160-169.

Donohue, M. C., R. A. Sperling, D. P. Salmon, D. M. Rentz, R. Raman, R. G. Thomas, M. Weiner, P. S. Aisen, B. Australian Imaging, A. Lifestyle Flagship Study of, I. Alzheimer's Disease Neuroimaging and S. Alzheimer's Disease Cooperative (2014). "The preclinical 
Alzheimer cognitive composite: measuring amyloid-related decline." JAMA Neurol 71(8): 961-970.

Fortea, J., E. Vilaplana, D. Alcolea, M. Carmona-Iragui, M. B. Sanchez-Saudinos, I. Sala, S. Anton-Aguirre, S. Gonzalez, S. Medrano, J. Pegueroles, E. Morenas, J. Clarimon, R. Blesa, A. Lleo and I. Alzheimer's Disease Neuroimaging (2014). "Cerebrospinal fluid beta-amyloid and phospho-tau biomarker interactions affecting brain structure in preclinical Alzheimer disease." Ann Neurol 76(2): 223-230.

Harrington, K. D., E. Gould, Y. Y. Lim, D. Ames, R. H. Pietrzak, A. Rembach, S. RaineySmith, R. N. Martins, O. Salvado, V. L. Villemagne, C. C. Rowe, C. L. Masters, P. Maruff and A. R. Group (2016). "Amyloid burden and incident depressive symptoms in cognitively normal older adults." Int J Geriatr Psychiatry.

Harrington, M. G., J. Chiang, J. M. Pogoda, M. Gomez, K. Thomas, S. D. Marion, K. J. Miller, P. Siddarth, X. Yi, F. Zhou, S. Lee, X. Arakaki, R. P. Cowan, T. Tran, C. Charleswell, B. D. Ross and A. N. Fonteh (2013). "Executive function changes before memory in preclinical Alzheimer's pathology: a prospective, cross-sectional, case control study." PLoS One 8(11): e79378.

Hassenstab, J., R. Chasse, P. Grabow, T. L. Benzinger, A. M. Fagan, C. Xiong, M. Jasielec, E. Grant and J. C. Morris (2016). "Certified normal: Alzheimer's disease biomarkers and normative estimates of cognitive functioning." Neurobiol Aging 43: 23-33.

Jack, C. R., Jr., D. S. Knopman, S. D. Weigand, H. J. Wiste, P. Vemuri, V. Lowe, K. Kantarci, J. L. Gunter, M. L. Senjem, R. J. Ivnik, R. O. Roberts, W. A. Rocca, B. F. Boeve and R. C. Petersen (2012). "An operational approach to National Institute on Aging-Alzheimer's Association criteria for preclinical Alzheimer disease." Ann Neurol 71(6): 765-775.

Jack, C. R., Jr., H. J. Wiste, S. D. Weigand, D. S. Knopman, M. M. Mielke, P. Vemuri, V. Lowe, M. L. Senjem, J. L. Gunter, D. Reyes, M. M. Machulda, R. Roberts and R. C. Petersen (2015). "Different definitions of neurodegeneration produce similar amyloid/neurodegeneration biomarker group findings." Brain 138(Pt 12): 3747-3759.

Jack, C. R., Jr., H. J. Wiste, S. D. Weigand, W. A. Rocca, D. S. Knopman, M. M. Mielke, V. J. Lowe, M. L. Senjem, J. L. Gunter, G. M. Preboske, V. S. Pankratz, P. Vemuri and R. C. Petersen (2014). "Age-specific population frequencies of cerebral beta-amyloidosis and neurodegeneration among people with normal cognitive function aged 50-89 years: a crosssectional study." Lancet Neurol 13(10): 997-1005.

Jicha, G. A., E. L. Abner, F. A. Schmitt, R. J. Kryscio, K. P. Riley, G. E. Cooper, N. Stiles, M. S. Mendiondo, C. D. Smith, L. J. Van Eldik and P. T. Nelson (2012). "Preclinical AD Workgroup staging: pathological correlates and potential challenges." Neurobiol Aging 33(3): 622 e621-622 e616.

Ju, Y. E., J. S. McLeland, C. D. Toedebusch, C. Xiong, A. M. Fagan, S. P. Duntley, J. C. Morris and D. M. Holtzman (2013). "Sleep quality and preclinical Alzheimer disease." JAMA Neurol 70(5): 587-593.

Knopman, D. S., C. R. Jack, Jr., H. J. Wiste, S. D. Weigand, P. Vemuri, V. Lowe, K. Kantarci, J. L. Gunter, M. L. Senjem, R. J. Ivnik, R. O. Roberts, B. F. Boeve and R. C. Petersen (2012). "Short-term clinical outcomes for stages of NIA-AA preclinical Alzheimer disease." Neurology 78(20): 1576-1582.

Knopman, D. S., C. R. Jack, Jr., H. J. Wiste, S. D. Weigand, P. Vemuri, V. J. Lowe, K. Kantarci, J. L. Gunter, M. L. Senjem, M. M. Mielke, R. O. Roberts, B. F. Boeve and R. C. Petersen (2013). "Brain injury biomarkers are not dependent on beta-amyloid in normal elderly." Ann Neurol 73(4): 472-480.

Lim, Y. Y., P. Maruff, R. H. Pietrzak, D. Ames, K. A. Ellis, K. Harrington, N. T. Lautenschlager, C. Szoeke, R. N. Martins, C. L. Masters, V. L. Villemagne, C. C. Rowe and 
A. R. Group (2014). "Effect of amyloid on memory and non-memory decline from preclinical to clinical Alzheimer's disease." Brain 137(Pt 1): 221-231.

Lim, Y. Y., P. J. Snyder, R. H. Pietrzak, A. Ukiqi, V. L. Villemagne, D. Ames, O. Salvado, P. Bourgeat, R. N. Martins, C. L. Masters, C. C. Rowe and P. Maruff (2016). "Sensitivity of composite scores to amyloid burden in preclinical Alzheimer's disease: Introducing the Zscores of Attention, Verbal fluency, and Episodic memory for Nondemented older adults composite score." Alzheimers Dement (Amst) 2: 19-26.

Lim, Y. Y., V. L. Villemagne, S. M. Laws, D. Ames, R. H. Pietrzak, K. A. Ellis, K. D. Harrington, P. Bourgeat, O. Salvado, D. Darby, P. J. Snyder, A. I. Bush, R. N. Martins, C. L. Masters, C. C. Rowe, P. J. Nathan, P. Maruff, B. Australian Imaging and G. Lifestyle Research (2013). "BDNF Val66Met, Abeta amyloid, and cognitive decline in preclinical Alzheimer's disease." Neurobiol Aging 34(11): 2457-2464.

Mielke, M. M., H. J. Wiste, S. D. Weigand, D. S. Knopman, V. J. Lowe, R. O. Roberts, Y. E. Geda, D. M. Swenson-Dravis, B. F. Boeve, M. L. Senjem, P. Vemuri, R. C. Petersen and C. R. Jack, Jr. (2012). "Indicators of amyloid burden in a population-based study of cognitively normal elderly." Neurology 79(15): 1570-1577.

Mormino, E. C., R. A. Betensky, T. Hedden, A. P. Schultz, R. E. Amariglio, D. M. Rentz, K. A. Johnson and R. A. Sperling (2014). "Synergistic effect of beta-amyloid and neurodegeneration on cognitive decline in clinically normal individuals." JAMA Neurol 71(11): 1379-1385.

Mormino, E. C., R. A. Betensky, T. Hedden, A. P. Schultz, A. Ward, W. Huijbers, D. M. Rentz, K. A. Johnson, R. A. Sperling, I. Alzheimer's Disease Neuroimaging, B. Australian Imaging, A. Lifestyle Flagship Study of and S. Harvard Aging Brain (2014). "Amyloid and APOE epsilon4 interact to influence short-term decline in preclinical Alzheimer disease." Neurology 82(20): 1760-1767.

Morris, J. C., C. M. Roe, E. A. Grant, D. Head, M. Storandt, A. M. Goate, A. M. Fagan, D. M. Holtzman and M. A. Mintun (2009). "Pittsburgh Compound B imaging and prediction of progression from cognitive normality to symptomatic Alzheimer disease." Archives of Neurology 66(12): 1469-1475.

Morris, J. C., C. M. Roe, C. Xiong, A. M. Fagan, A. M. Goate, D. M. Holtzman and M. A. Mintun (2010). "APOE predicts amyloid-beta but not tau Alzheimer pathology in cognitively normal aging." Ann Neurol 67(1): 122-131.

Nation, D. A., E. C. Edmonds, K. J. Bangen, L. Delano-Wood, B. K. Scanlon, S. D. Han, S. D. Edland, D. P. Salmon, D. R. Galasko, M. W. Bondi and I. Alzheimer's Disease Neuroimaging Initiative (2015). "Pulse pressure in relation to tau-mediated neurodegeneration, cerebral amyloidosis, and progression to dementia in very old adults." JAMA Neurol 72(5): 546-553. Papp, K. V., R. E. Amariglio, E. C. Mormino, T. Hedden, M. Dekhytar, K. A. Johnson, R. A. Sperling and D. M. Rentz (2015). "Free and cued memory in relation to biomarker-defined abnormalities in clinically normal older adults and those at risk for Alzheimer's disease." Neuropsychologia 73: 169-175.

Papp, K. V., E. C. Mormino, R. E. Amariglio, C. Munro, A. Dagley, A. P. Schultz, K. A. Johnson, R. A. Sperling and D. M. Rentz (2016). "Biomarker validation of a decline in semantic processing in preclinical Alzheimer's disease." Neuropsychology 30(5): 624-630. Pascoal, T. A., S. Mathotaarachchi, S. Mohades, A. L. Benedet, C. O. Chung, M. Shin, S. Wang, T. Beaudry, M. S. Kang, J. P. Soucy, A. Labbe, S. Gauthier and P. Rosa-Neto (2016). "Amyloid-beta and hyperphosphorylated tau synergy drives metabolic decline in preclinical Alzheimer's disease." Mol Psychiatry.

Pettigrew, C., A. Soldan, A. Moghekar, M. C. Wang, A. L. Gross, R. O'Brien and M. Albert (2015). "Relationship between cerebrospinal fluid biomarkers of Alzheimer's disease and cognition in cognitively normal older adults." Neuropsychologia 78: 63-72. 
Pietrzak, R. H., Y. Y. Lim, D. Ames, K. Harrington, C. Restrepo, R. N. Martins, A. Rembach, S. M. Laws, C. L. Masters, V. L. Villemagne, C. C. Rowe, P. Maruff, B. Australian Imaging and G. Lifestyle Research (2015). "Trajectories of memory decline in preclinical Alzheimer's disease: results from the Australian Imaging, Biomarkers and Lifestyle Flagship Study of ageing." Neurobiol Aging 36(3): 1231-1238.

Pietrzak, R. H., Y. Y. Lim, A. Neumeister, D. Ames, K. A. Ellis, K. Harrington, N. T. Lautenschlager, C. Restrepo, R. N. Martins, C. L. Masters, V. L. Villemagne, C. C. Rowe, P. Maruff, B. Australian Imaging and G. Lifestyle Research (2015). "Amyloid-beta, anxiety, and cognitive decline in preclinical Alzheimer disease: a multicenter, prospective cohort study." JAMA Psychiatry 72(3): 284-291.

Pike, K. E., K. A. Ellis, V. L. Villemagne, N. Good, G. Chetelat, D. Ames, C. Szoeke, S. M. Laws, G. Verdile, R. N. Martins, C. L. Masters and C. C. Rowe (2011). "Cognition and betaamyloid in preclinical Alzheimer's disease: data from the AIBL study." Neuropsychologia 49(9): 2384-2390.

Racine, A. M., N. Adluru, A. L. Alexander, B. T. Christian, O. C. Okonkwo, J. Oh, C. A. Cleary, A. Birdsill, A. T. Hillmer, D. Murali, T. E. Barnhart, C. L. Gallagher, C. M. Carlsson, H. A. Rowley, N. M. Dowling, S. Asthana, M. A. Sager, B. B. Bendlin and S. C. Johnson (2014). "Associations between white matter microstructure and amyloid burden in preclinical Alzheimer's disease: A multimodal imaging investigation." Neuroimage Clin 4: 604-614.

Racine, A. M., R. L. Koscik, S. E. Berman, C. R. Nicholas, L. R. Clark, O. C. Okonkwo, H. A. Rowley, S. Asthana, B. B. Bendlin, K. Blennow, H. Zetterberg, C. E. Gleason, C. M. Carlsson and S. C. Johnson (2016). "Biomarker clusters are differentially associated with longitudinal cognitive decline in late midlife." Brain.

Riley, K. P., G. A. Jicha, D. Davis, E. L. Abner, G. E. Cooper, N. Stiles, C. D. Smith, R. J. Kryscio, P. T. Nelson, L. J. Van Eldik and F. A. Schmitt (2011). "Prediction of preclinical Alzheimer's disease: longitudinal rates of change in cognition." J Alzheimers Dis 25(4): 707717.

Soldan, A., C. Pettigrew, Q. Cai, M. C. Wang, A. R. Moghekar, R. J. O'Brien, O. A. Selnes, M. S. Albert and B. R. Team (2016). "Hypothetical Preclinical Alzheimer Disease Groups and Longitudinal Cognitive Change." JAMA Neurol 73(6): 698-705.

Stark, S. L., C. M. Roe, E. A. Grant, H. Hollingsworth, T. L. Benzinger, A. M. Fagan, V. D. Buckles and J. C. Morris (2013). "Preclinical Alzheimer disease and risk of falls." Neurology 81(5): 437-443.

Sutphen, C. L., M. S. Jasielec, A. R. Shah, E. M. Macy, C. Xiong, A. G. Vlassenko, T. L. Benzinger, E. E. Stoops, H. M. Vanderstichele, B. Brix, H. D. Darby, M. L. Vandijck, J. H. Ladenson, J. C. Morris, D. M. Holtzman and A. M. Fagan (2015). "Longitudinal Cerebrospinal Fluid Biomarker Changes in Preclinical Alzheimer Disease During Middle Age." JAMA Neurol 72(9): 1029-1042.

Thai, C., Y. Y. Lim, V. L. Villemagne, S. M. Laws, D. Ames, K. A. Ellis, S. R. Rainey-Smith, R. N. Martins, C. L. Masters, C. C. Rowe, P. Maruff, B. Australian Imaging and G. Lifestyle Research (2015). "Amyloid-Related Memory Decline in Preclinical Alzheimer's Disease Is Dependent on APOE epsilon4 and Is Detectable over 18-Months." PLoS One 10(10): e0139082.

Thal, D. R., C. von Arnim, W. S. Griffin, H. Yamaguchi, R. E. Mrak, J. Attems and A. R. Upadhaya (2013). "Pathology of clinical and preclinical Alzheimer's disease." Eur Arch Psychiatry Clin Neurosci 263 Suppl 2: S137-145.

Valech, N., M. A. Mollica, J. Olives, A. Tort, J. Fortea, A. Lleo, S. S. Belen, J. L. Molinuevo and L. Rami (2015). "Informants' Perception of Subjective Cognitive Decline Helps to Discriminate Preclinical Alzheimer's Disease from Normal Aging." J Alzheimers Dis 48 Suppl 1: S87-98. 
van Harten, A. C., L. L. Smits, C. E. Teunissen, P. J. Visser, T. Koene, M. A. Blankenstein, P. Scheltens and W. M. van der Flier (2013). "Preclinical AD predicts decline in memory and executive functions in subjective complaints." Neurology 81(16): 1409-1416.

Voevodskaya, O., P. C. Sundgren, O. Strandberg, H. Zetterberg, L. Minthon, K. Blennow, L. O. Wahlund, E. Westman, O. Hansson and F. s. g. Swedish Bio (2016). "Myo-inositol changes precede amyloid pathology and relate to APOE genotype in Alzheimer disease." Neurology 86(19): 1754-1761.

Vos, S. J., B. A. Gordon, Y. Su, P. J. Visser, D. M. Holtzman, J. C. Morris, A. M. Fagan and T. L. Benzinger (2016). "NIA-AA staging of preclinical Alzheimer disease: discordance and concordance of CSF and imaging biomarkers." Neurobiol Aging 44: 1-8.

Vos, S. J., C. Xiong, P. J. Visser, M. S. Jasielec, J. Hassenstab, E. A. Grant, N. J. Cairns, J. C. Morris, D. M. Holtzman and A. M. Fagan (2013). "Preclinical Alzheimer's disease and its outcome: a longitudinal cohort study." Lancet Neurol 12(10): 957-965.

Wang, L., T. L. Benzinger, J. Hassenstab, T. Blazey, C. Owen, J. Liu, A. M. Fagan, J. C. Morris and B. M. Ances (2015). "Spatially distinct atrophy is linked to beta-amyloid and tau in preclinical Alzheimer disease." Neurology 84(12): 1254-1260.

Whitwell, J. L., N. Tosakulwong, S. D. Weigand, M. L. Senjem, V. J. Lowe, J. L. Gunter, B. F. Boeve, D. S. Knopman, B. C. Dickerson, R. C. Petersen and C. R. Jack, Jr. (2013). "Does amyloid deposition produce a specific atrophic signature in cognitively normal subjects?" Neuroimage Clin 2: 249-257.

Abner, E. L., R. J. Kryscio, F. A. Schmitt, K. S. Santacruz, G. A. Jicha, Y. Lin, J. M. Neltner, C. D. Smith, L. J. Van Eldik and P. T. Nelson (2011). ""End-stage" neurofibrillary tangle pathology in preclinical Alzheimer's disease: fact or fiction?" J Alzheimers Dis 25(3): 445453.

Alcolea, D., P. Martinez-Lage, P. Sanchez-Juan, J. Olazaran, C. Antunez, A. Izagirre, M. Ecay-Torres, A. Estanga, M. Clerigue, M. C. Guisasola, D. Sanchez Ruiz, J. Marin Munoz, M. Calero, R. Blesa, J. Clarimon, M. Carmona-Iragui, E. Morenas-Rodriguez, E. RodriguezRodriguez, J. L. Vazquez Higuera, J. Fortea and A. Lleo (2015). "Amyloid precursor protein metabolism and inflammation markers in preclinical Alzheimer disease." Neurology 85(7): 626-633.

Amariglio, R. E., J. A. Becker, J. Carmasin, L. P. Wadsworth, N. Lorius, C. Sullivan, J. E. Maye, C. Gidicsin, L. C. Pepin, R. A. Sperling, K. A. Johnson and D. M. Rentz (2012).

"Subjective cognitive complaints and amyloid burden in cognitively normal older individuals." Neuropsychologia 50(12): 2880-2886.

Ayutyanont, N., J. B. Langbaum, S. B. Hendrix, K. Chen, A. S. Fleisher, M. Friesenhahn, M. Ward, C. Aguirre, N. Acosta-Baena, L. Madrigal, C. Munoz, V. Tirado, S. Moreno, P. N. Tariot, F. Lopera and E. M. Reiman (2014). "The Alzheimer's prevention initiative composite cognitive test score: sample size estimates for the evaluation of preclinical Alzheimer's disease treatments in presenilin 1 E280A mutation carriers." J Clin Psychiatry 75(6): 652-660. Bilgel, M., J. L. Prince, D. F. Wong, S. M. Resnick and B. M. Jedynak (2016). "A multivariate nonlinear mixed effects model for longitudinal image analysis: Application to amyloid imaging." Neuroimage 134: 658-670.

Brier, M. R., J. E. McCarthy, T. L. Benzinger, A. Stern, Y. Su, K. A. Friedrichsen, J. C. Morris, B. M. Ances and A. G. Vlassenko (2016). "Local and distributed PiB accumulation associated with development of preclinical Alzheimer's disease." Neurobiol Aging 38: 104111.

Brier, M. R., J. B. Thomas, A. M. Fagan, J. Hassenstab, D. M. Holtzman, T. L. Benzinger, J. C. Morris and B. M. Ances (2014). "Functional connectivity and graph theory in preclinical Alzheimer's disease." Neurobiol Aging 35(4): 757-768. 
Brier, M. R., J. B. Thomas, A. Z. Snyder, L. Wang, A. M. Fagan, T. Benzinger, J. C. Morris and B. M. Ances (2014). "Unrecognized preclinical Alzheimer disease confounds rs-fcMRI studies of normal aging." Neurology 83(18): 1613-1619.

Clark, L. R., A. M. Racine, R. L. Koscik, O. C. Okonkwo, C. D. Engelman, C. M. Carlsson, S. Asthana, B. B. Bendlin, R. Chappell, C. R. Nicholas, H. A. Rowley, J. M. Oh, B. P. Hermann, M. A. Sager, B. T. Christian and S. C. Johnson (2016). "Beta-amyloid and cognitive decline in late middle age: Findings from the Wisconsin Registry for Alzheimer's Prevention study." Alzheimers Dement.

Craig-Schapiro, R., R. J. Perrin, C. M. Roe, C. Xiong, D. Carter, N. J. Cairns, M. A. Mintun, E. R. Peskind, G. Li, D. R. Galasko, C. M. Clark, J. F. Quinn, G. D'Angelo, J. P. Malone, R. R. Townsend, J. C. Morris, A. M. Fagan and D. M. Holtzman (2010). "YKL-40: a novel prognostic fluid biomarker for preclinical Alzheimer's disease." Biol Psychiatry 68(10): 903912.

Desikan, R. S., L. K. McEvoy, W. K. Thompson, D. Holland, J. B. Brewer, P. S. Aisen, R. A. Sperling, A. M. Dale and I. Alzheimer's Disease Neuroimaging (2012). "Amyloid-beta-associated clinical decline occurs only in the presence of elevated P-tau." Arch Neurol 69(6): 709-713.

Doherty, B. M., S. A. Schultz, J. M. Oh, R. L. Koscik, N. M. Dowling, T. E. Barnhart, D. Murali, C. L. Gallagher, C. M. Carlsson, B. B. Bendlin, A. LaRue, B. P. Hermann, H. A. Rowley, S. Asthana, M. A. Sager, B. T. Christian, S. C. Johnson and O. C. Okonkwo (2015). "Amyloid burden, cortical thickness, and cognitive function in the Wisconsin Registry for Alzheimer's Prevention." Alzheimers Dement (Amst) 1(2): 160-169.

Donohue, M. C., R. A. Sperling, D. P. Salmon, D. M. Rentz, R. Raman, R. G. Thomas, M. Weiner, P. S. Aisen, B. Australian Imaging, A. Lifestyle Flagship Study of, I. Alzheimer's Disease Neuroimaging and S. Alzheimer's Disease Cooperative (2014). "The preclinical Alzheimer cognitive composite: measuring amyloid-related decline." JAMA Neurol 71(8): 961-970.

Fortea, J., E. Vilaplana, D. Alcolea, M. Carmona-Iragui, M. B. Sanchez-Saudinos, I. Sala, S. Anton-Aguirre, S. Gonzalez, S. Medrano, J. Pegueroles, E. Morenas, J. Clarimon, R. Blesa, A. Lleo and I. Alzheimer's Disease Neuroimaging (2014). "Cerebrospinal fluid beta-amyloid and phospho-tau biomarker interactions affecting brain structure in preclinical Alzheimer disease." Ann Neurol 76(2): 223-230.

Harrington, K. D., E. Gould, Y. Y. Lim, D. Ames, R. H. Pietrzak, A. Rembach, S. RaineySmith, R. N. Martins, O. Salvado, V. L. Villemagne, C. C. Rowe, C. L. Masters, P. Maruff and A. R. Group (2016). "Amyloid burden and incident depressive symptoms in cognitively normal older adults." Int J Geriatr Psychiatry.

Harrington, M. G., J. Chiang, J. M. Pogoda, M. Gomez, K. Thomas, S. D. Marion, K. J. Miller, P. Siddarth, X. Yi, F. Zhou, S. Lee, X. Arakaki, R. P. Cowan, T. Tran, C. Charleswell, B. D. Ross and A. N. Fonteh (2013). "Executive function changes before memory in preclinical Alzheimer's pathology: a prospective, cross-sectional, case control study." PLoS One 8(11): e79378.

Jack, C. R., Jr., D. S. Knopman, S. D. Weigand, H. J. Wiste, P. Vemuri, V. Lowe, K. Kantarci, J. L. Gunter, M. L. Senjem, R. J. Ivnik, R. O. Roberts, W. A. Rocca, B. F. Boeve and R. C. Petersen (2012). "An operational approach to National Institute on Aging-Alzheimer's Association criteria for preclinical Alzheimer disease." Ann Neurol 71(6): 765-775. Jack, C. R., Jr., H. J. Wiste, S. D. Weigand, D. S. Knopman, M. M. Mielke, P. Vemuri, V. Lowe, M. L. Senjem, J. L. Gunter, D. Reyes, M. M. Machulda, R. Roberts and R. C. Petersen (2015). "Different definitions of neurodegeneration produce similar amyloid/neurodegeneration biomarker group findings." Brain 138(Pt 12): 3747-3759. 
Jack, C. R., Jr., H. J. Wiste, S. D. Weigand, W. A. Rocca, D. S. Knopman, M. M. Mielke, V. J. Lowe, M. L. Senjem, J. L. Gunter, G. M. Preboske, V. S. Pankratz, P. Vemuri and R. C. Petersen (2014). "Age-specific population frequencies of cerebral beta-amyloidosis and neurodegeneration among people with normal cognitive function aged 50-89 years: a crosssectional study." Lancet Neurol 13(10): 997-1005.

Jicha, G. A., E. L. Abner, F. A. Schmitt, R. J. Kryscio, K. P. Riley, G. E. Cooper, N. Stiles, M. S. Mendiondo, C. D. Smith, L. J. Van Eldik and P. T. Nelson (2012). "Preclinical AD Workgroup staging: pathological correlates and potential challenges." Neurobiol Aging 33(3): 622 e621-622 e616.

Ju, Y. E., J. S. McLeland, C. D. Toedebusch, C. Xiong, A. M. Fagan, S. P. Duntley, J. C. Morris and D. M. Holtzman (2013). "Sleep quality and preclinical Alzheimer disease." JAMA Neurol 70(5): 587-593.

Knopman, D. S., C. R. Jack, Jr., H. J. Wiste, S. D. Weigand, P. Vemuri, V. Lowe, K. Kantarci, J. L. Gunter, M. L. Senjem, R. J. Ivnik, R. O. Roberts, B. F. Boeve and R. C. Petersen (2012). "Short-term clinical outcomes for stages of NIA-AA preclinical Alzheimer disease." Neurology 78(20): 1576-1582.

Knopman, D. S., C. R. Jack, Jr., H. J. Wiste, S. D. Weigand, P. Vemuri, V. J. Lowe, K. Kantarci, J. L. Gunter, M. L. Senjem, M. M. Mielke, R. O. Roberts, B. F. Boeve and R. C. Petersen (2013). "Brain injury biomarkers are not dependent on beta-amyloid in normal elderly." Ann Neurol 73(4): 472-480.

Lim, Y. Y., P. Maruff, R. H. Pietrzak, D. Ames, K. A. Ellis, K. Harrington, N. T.

Lautenschlager, C. Szoeke, R. N. Martins, C. L. Masters, V. L. Villemagne, C. C. Rowe and A. R. Group (2014). "Effect of amyloid on memory and non-memory decline from preclinical to clinical Alzheimer's disease." Brain 137(Pt 1): 221-231.

Lim, Y. Y., P. J. Snyder, R. H. Pietrzak, A. Ukiqi, V. L. Villemagne, D. Ames, O. Salvado, P. Bourgeat, R. N. Martins, C. L. Masters, C. C. Rowe and P. Maruff (2016). "Sensitivity of composite scores to amyloid burden in preclinical Alzheimer's disease: Introducing the Zscores of Attention, Verbal fluency, and Episodic memory for Nondemented older adults composite score." Alzheimers Dement (Amst) 2: 19-26.

Lim, Y. Y., V. L. Villemagne, S. M. Laws, D. Ames, R. H. Pietrzak, K. A. Ellis, K. D. Harrington, P. Bourgeat, O. Salvado, D. Darby, P. J. Snyder, A. I. Bush, R. N. Martins, C. L. Masters, C. C. Rowe, P. J. Nathan, P. Maruff, B. Australian Imaging and G. Lifestyle Research (2013). "BDNF Val66Met, Abeta amyloid, and cognitive decline in preclinical Alzheimer's disease." Neurobiol Aging 34(11): 2457-2464.

Mielke, M. M., H. J. Wiste, S. D. Weigand, D. S. Knopman, V. J. Lowe, R. O. Roberts, Y. E. Geda, D. M. Swenson-Dravis, B. F. Boeve, M. L. Senjem, P. Vemuri, R. C. Petersen and C. R. Jack, Jr. (2012). "Indicators of amyloid burden in a population-based study of cognitively normal elderly." Neurology 79(15): 1570-1577.

Mormino, E. C., R. A. Betensky, T. Hedden, A. P. Schultz, R. E. Amariglio, D. M. Rentz, K. A. Johnson and R. A. Sperling (2014). "Synergistic effect of beta-amyloid and neurodegeneration on cognitive decline in clinically normal individuals." JAMA Neurol 71(11): 1379-1385.

Mormino, E. C., R. A. Betensky, T. Hedden, A. P. Schultz, A. Ward, W. Huijbers, D. M. Rentz, K. A. Johnson, R. A. Sperling, I. Alzheimer's Disease Neuroimaging, B. Australian Imaging, A. Lifestyle Flagship Study of and S. Harvard Aging Brain (2014). "Amyloid and APOE epsilon4 interact to influence short-term decline in preclinical Alzheimer disease." Neurology 82(20): 1760-1767.

Morris, J. C., C. M. Roe, E. A. Grant, D. Head, M. Storandt, A. M. Goate, A. M. Fagan, D. M. Holtzman and M. A. Mintun (2009). "Pittsburgh Compound B imaging and prediction of 
progression from cognitive normality to symptomatic Alzheimer disease." Archives of Neurology 66(12): 1469-1475.

Morris, J. C., C. M. Roe, C. Xiong, A. M. Fagan, A. M. Goate, D. M. Holtzman and M. A. Mintun (2010). "APOE predicts amyloid-beta but not tau Alzheimer pathology in cognitively normal aging." Ann Neurol 67(1): 122-131.

Nation, D. A., E. C. Edmonds, K. J. Bangen, L. Delano-Wood, B. K. Scanlon, S. D. Han, S. D. Edland, D. P. Salmon, D. R. Galasko, M. W. Bondi and I. Alzheimer's Disease Neuroimaging Initiative (2015). "Pulse pressure in relation to tau-mediated neurodegeneration, cerebral amyloidosis, and progression to dementia in very old adults." JAMA Neurol 72(5): 546-553. Papp, K. V., R. E. Amariglio, E. C. Mormino, T. Hedden, M. Dekhytar, K. A. Johnson, R. A. Sperling and D. M. Rentz (2015). "Free and cued memory in relation to biomarker-defined abnormalities in clinically normal older adults and those at risk for Alzheimer's disease." Neuropsychologia 73: 169-175.

Papp, K. V., E. C. Mormino, R. E. Amariglio, C. Munro, A. Dagley, A. P. Schultz, K. A. Johnson, R. A. Sperling and D. M. Rentz (2016). "Biomarker validation of a decline in semantic processing in preclinical Alzheimer's disease." Neuropsychology 30(5): 624-630. Pascoal, T. A., S. Mathotaarachchi, S. Mohades, A. L. Benedet, C. O. Chung, M. Shin, S. Wang, T. Beaudry, M. S. Kang, J. P. Soucy, A. Labbe, S. Gauthier and P. Rosa-Neto (2016). "Amyloid-beta and hyperphosphorylated tau synergy drives metabolic decline in preclinical Alzheimer's disease." Mol Psychiatry.

Pettigrew, C., A. Soldan, A. Moghekar, M. C. Wang, A. L. Gross, R. O'Brien and M. Albert (2015). "Relationship between cerebrospinal fluid biomarkers of Alzheimer's disease and cognition in cognitively normal older adults." Neuropsychologia 78: 63-72.

Pietrzak, R. H., Y. Y. Lim, D. Ames, K. Harrington, C. Restrepo, R. N. Martins, A. Rembach, S. M. Laws, C. L. Masters, V. L. Villemagne, C. C. Rowe, P. Maruff, B. Australian Imaging and G. Lifestyle Research (2015). "Trajectories of memory decline in preclinical Alzheimer's disease: results from the Australian Imaging, Biomarkers and Lifestyle Flagship Study of ageing." Neurobiol Aging 36(3): 1231-1238.

Pietrzak, R. H., Y. Y. Lim, A. Neumeister, D. Ames, K. A. Ellis, K. Harrington, N. T. Lautenschlager, C. Restrepo, R. N. Martins, C. L. Masters, V. L. Villemagne, C. C. Rowe, P. Maruff, B. Australian Imaging and G. Lifestyle Research (2015). "Amyloid-beta, anxiety, and cognitive decline in preclinical Alzheimer disease: a multicenter, prospective cohort study." JAMA Psychiatry 72(3): 284-291.

Pike, K. E., K. A. Ellis, V. L. Villemagne, N. Good, G. Chetelat, D. Ames, C. Szoeke, S. M. Laws, G. Verdile, R. N. Martins, C. L. Masters and C. C. Rowe (2011). "Cognition and betaamyloid in preclinical Alzheimer's disease: data from the AIBL study." Neuropsychologia 49(9): 2384-2390.

Racine, A. M., N. Adluru, A. L. Alexander, B. T. Christian, O. C. Okonkwo, J. Oh, C. A. Cleary, A. Birdsill, A. T. Hillmer, D. Murali, T. E. Barnhart, C. L. Gallagher, C. M. Carlsson, H. A. Rowley, N. M. Dowling, S. Asthana, M. A. Sager, B. B. Bendlin and S. C. Johnson (2014). "Associations between white matter microstructure and amyloid burden in preclinical Alzheimer's disease: A multimodal imaging investigation." Neuroimage Clin 4: 604-614.

Racine, A. M., R. L. Koscik, S. E. Berman, C. R. Nicholas, L. R. Clark, O. C. Okonkwo, H. A. Rowley, S. Asthana, B. B. Bendlin, K. Blennow, H. Zetterberg, C. E. Gleason, C. M. Carlsson and S. C. Johnson (2016). "Biomarker clusters are differentially associated with longitudinal cognitive decline in late midlife." Brain.

Riley, K. P., G. A. Jicha, D. Davis, E. L. Abner, G. E. Cooper, N. Stiles, C. D. Smith, R. J. Kryscio, P. T. Nelson, L. J. Van Eldik and F. A. Schmitt (2011). "Prediction of preclinical Alzheimer's disease: longitudinal rates of change in cognition." J Alzheimers Dis 25(4): 707717. 
Soldan, A., C. Pettigrew, Q. Cai, M. C. Wang, A. R. Moghekar, R. J. O'Brien, O. A. Selnes, M. S. Albert and B. R. Team (2016). "Hypothetical Preclinical Alzheimer Disease Groups and Longitudinal Cognitive Change." JAMA Neurol 73(6): 698-705.

Stark, S. L., C. M. Roe, E. A. Grant, H. Hollingsworth, T. L. Benzinger, A. M. Fagan, V. D. Buckles and J. C. Morris (2013). "Preclinical Alzheimer disease and risk of falls." Neurology 81(5): 437-443.

Sutphen, C. L., M. S. Jasielec, A. R. Shah, E. M. Macy, C. Xiong, A. G. Vlassenko, T. L. Benzinger, E. E. Stoops, H. M. Vanderstichele, B. Brix, H. D. Darby, M. L. Vandijck, J. H. Ladenson, J. C. Morris, D. M. Holtzman and A. M. Fagan (2015). "Longitudinal Cerebrospinal Fluid Biomarker Changes in Preclinical Alzheimer Disease During Middle Age." JAMA Neurol 72(9): 1029-1042.

Thai, C., Y. Y. Lim, V. L. Villemagne, S. M. Laws, D. Ames, K. A. Ellis, S. R. Rainey-Smith, R. N. Martins, C. L. Masters, C. C. Rowe, P. Maruff, B. Australian Imaging and G. Lifestyle Research (2015). "Amyloid-Related Memory Decline in Preclinical Alzheimer's Disease Is Dependent on APOE epsilon4 and Is Detectable over 18-Months." PLoS One 10(10): e0139082.

Thal, D. R., C. von Arnim, W. S. Griffin, H. Yamaguchi, R. E. Mrak, J. Attems and A. R. Upadhaya (2013). "Pathology of clinical and preclinical Alzheimer's disease." Eur Arch Psychiatry Clin Neurosci 263 Suppl 2: S137-145.

Valech, N., M. A. Mollica, J. Olives, A. Tort, J. Fortea, A. Lleo, S. S. Belen, J. L. Molinuevo and L. Rami (2015). "Informants' Perception of Subjective Cognitive Decline Helps to Discriminate Preclinical Alzheimer's Disease from Normal Aging." J Alzheimers Dis 48 Suppl 1: S87-98.

van Harten, A. C., L. L. Smits, C. E. Teunissen, P. J. Visser, T. Koene, M. A. Blankenstein, P. Scheltens and W. M. van der Flier (2013). "Preclinical AD predicts decline in memory and executive functions in subjective complaints." Neurology 81(16): 1409-1416.

Voevodskaya, O., P. C. Sundgren, O. Strandberg, H. Zetterberg, L. Minthon, K. Blennow, L. O. Wahlund, E. Westman, O. Hansson and F. s. g. Swedish Bio (2016). "Myo-inositol changes precede amyloid pathology and relate to APOE genotype in Alzheimer disease." Neurology 86(19): 1754-1761.

Vos, S. J., B. A. Gordon, Y. Su, P. J. Visser, D. M. Holtzman, J. C. Morris, A. M. Fagan and T. L. Benzinger (2016). "NIA-AA staging of preclinical Alzheimer disease: discordance and concordance of CSF and imaging biomarkers." Neurobiol Aging 44: 1-8.

Vos, S. J., C. Xiong, P. J. Visser, M. S. Jasielec, J. Hassenstab, E. A. Grant, N. J. Cairns, J. C. Morris, D. M. Holtzman and A. M. Fagan (2013). "Preclinical Alzheimer's disease and its outcome: a longitudinal cohort study." Lancet Neurol 12(10): 957-965.

Wang, L., T. L. Benzinger, J. Hassenstab, T. Blazey, C. Owen, J. Liu, A. M. Fagan, J. C. Morris and B. M. Ances (2015). "Spatially distinct atrophy is linked to beta-amyloid and tau in preclinical Alzheimer disease." Neurology 84(12): 1254-1260.

Whitwell, J. L., N. Tosakulwong, S. D. Weigand, M. L. Senjem, V. J. Lowe, J. L. Gunter, B. F. Boeve, D. S. Knopman, B. C. Dickerson, R. C. Petersen and C. R. Jack, Jr. (2013). "Does amyloid deposition produce a specific atrophic signature in cognitively normal subjects?" Neuroimage Clin 2: 249-257.

Abner, E. L., R. J. Kryscio, F. A. Schmitt, K. S. Santacruz, G. A. Jicha, Y. Lin, J. M. Neltner, C. D. Smith, L. J. Van Eldik and P. T. Nelson (2011). ""End-stage" neurofibrillary tangle pathology in preclinical Alzheimer's disease: fact or fiction?" J Alzheimers Dis 25(3): 445453.

Alcolea, D., P. Martinez-Lage, P. Sanchez-Juan, J. Olazaran, C. Antunez, A. Izagirre, M. Ecay-Torres, A. Estanga, M. Clerigue, M. C. Guisasola, D. Sanchez Ruiz, J. Marin Munoz, M. Calero, R. Blesa, J. Clarimon, M. Carmona-Iragui, E. Morenas-Rodriguez, E. Rodriguez- 
Rodriguez, J. L. Vazquez Higuera, J. Fortea and A. Lleo (2015). "Amyloid precursor protein metabolism and inflammation markers in preclinical Alzheimer disease." Neurology 85(7): 626-633.

Amariglio, R. E., J. A. Becker, J. Carmasin, L. P. Wadsworth, N. Lorius, C. Sullivan, J. E. Maye, C. Gidicsin, L. C. Pepin, R. A. Sperling, K. A. Johnson and D. M. Rentz (2012).

"Subjective cognitive complaints and amyloid burden in cognitively normal older individuals." Neuropsychologia 50(12): 2880-2886.

Ayutyanont, N., J. B. Langbaum, S. B. Hendrix, K. Chen, A. S. Fleisher, M. Friesenhahn, M. Ward, C. Aguirre, N. Acosta-Baena, L. Madrigal, C. Munoz, V. Tirado, S. Moreno, P. N. Tariot, F. Lopera and E. M. Reiman (2014). "The Alzheimer's prevention initiative composite cognitive test score: sample size estimates for the evaluation of preclinical Alzheimer's disease treatments in presenilin 1 E280A mutation carriers." J Clin Psychiatry 75(6): 652-660. Bilgel, M., J. L. Prince, D. F. Wong, S. M. Resnick and B. M. Jedynak (2016). "A multivariate nonlinear mixed effects model for longitudinal image analysis: Application to amyloid imaging." Neuroimage 134: 658-670.

Brier, M. R., J. E. McCarthy, T. L. Benzinger, A. Stern, Y. Su, K. A. Friedrichsen, J. C. Morris, B. M. Ances and A. G. Vlassenko (2016). "Local and distributed PiB accumulation associated with development of preclinical Alzheimer's disease." Neurobiol Aging 38: 104111.

Brier, M. R., J. B. Thomas, A. M. Fagan, J. Hassenstab, D. M. Holtzman, T. L. Benzinger, J. C. Morris and B. M. Ances (2014). "Functional connectivity and graph theory in preclinical Alzheimer's disease." Neurobiol Aging 35(4): 757-768.

Brier, M. R., J. B. Thomas, A. Z. Snyder, L. Wang, A. M. Fagan, T. Benzinger, J. C. Morris and B. M. Ances (2014). "Unrecognized preclinical Alzheimer disease confounds rs-fcMRI studies of normal aging." Neurology 83(18): 1613-1619.

Clark, L. R., A. M. Racine, R. L. Koscik, O. C. Okonkwo, C. D. Engelman, C. M. Carlsson, S. Asthana, B. B. Bendlin, R. Chappell, C. R. Nicholas, H. A. Rowley, J. M. Oh, B. P. Hermann, M. A. Sager, B. T. Christian and S. C. Johnson (2016). "Beta-amyloid and cognitive decline in late middle age: Findings from the Wisconsin Registry for Alzheimer's Prevention study." Alzheimers Dement.

Craig-Schapiro, R., R. J. Perrin, C. M. Roe, C. Xiong, D. Carter, N. J. Cairns, M. A. Mintun, E. R. Peskind, G. Li, D. R. Galasko, C. M. Clark, J. F. Quinn, G. D'Angelo, J. P. Malone, R. R. Townsend, J. C. Morris, A. M. Fagan and D. M. Holtzman (2010). "YKL-40: a novel prognostic fluid biomarker for preclinical Alzheimer's disease." Biol Psychiatry 68(10): 903912.

Desikan, R. S., L. K. McEvoy, W. K. Thompson, D. Holland, J. B. Brewer, P. S. Aisen, R. A. Sperling, A. M. Dale and I. Alzheimer's Disease Neuroimaging (2012). "Amyloid-beta-associated clinical decline occurs only in the presence of elevated P-tau." Arch Neurol 69(6): 709-713.

Doherty, B. M., S. A. Schultz, J. M. Oh, R. L. Koscik, N. M. Dowling, T. E. Barnhart, D. Murali, C. L. Gallagher, C. M. Carlsson, B. B. Bendlin, A. LaRue, B. P. Hermann, H. A. Rowley, S. Asthana, M. A. Sager, B. T. Christian, S. C. Johnson and O. C. Okonkwo (2015). "Amyloid burden, cortical thickness, and cognitive function in the Wisconsin Registry for Alzheimer's Prevention." Alzheimers Dement (Amst) 1(2): 160-169.

Donohue, M. C., R. A. Sperling, D. P. Salmon, D. M. Rentz, R. Raman, R. G. Thomas, M. Weiner, P. S. Aisen, B. Australian Imaging, A. Lifestyle Flagship Study of, I. Alzheimer's Disease Neuroimaging and S. Alzheimer's Disease Cooperative (2014). "The preclinical Alzheimer cognitive composite: measuring amyloid-related decline." JAMA Neurol 71(8): 961-970. 
Fortea, J., E. Vilaplana, D. Alcolea, M. Carmona-Iragui, M. B. Sanchez-Saudinos, I. Sala, S. Anton-Aguirre, S. Gonzalez, S. Medrano, J. Pegueroles, E. Morenas, J. Clarimon, R. Blesa, A. Lleo and I. Alzheimer's Disease Neuroimaging (2014). "Cerebrospinal fluid beta-amyloid and phospho-tau biomarker interactions affecting brain structure in preclinical Alzheimer disease." Ann Neurol 76(2): 223-230.

Harrington, K. D., E. Gould, Y. Y. Lim, D. Ames, R. H. Pietrzak, A. Rembach, S. RaineySmith, R. N. Martins, O. Salvado, V. L. Villemagne, C. C. Rowe, C. L. Masters, P. Maruff and A. R. Group (2016). "Amyloid burden and incident depressive symptoms in cognitively normal older adults." Int J Geriatr Psychiatry.

Harrington, M. G., J. Chiang, J. M. Pogoda, M. Gomez, K. Thomas, S. D. Marion, K. J. Miller, P. Siddarth, X. Yi, F. Zhou, S. Lee, X. Arakaki, R. P. Cowan, T. Tran, C. Charleswell, B. D. Ross and A. N. Fonteh (2013). "Executive function changes before memory in preclinical Alzheimer's pathology: a prospective, cross-sectional, case control study." PLoS One 8(11): e79378.

Hassenstab, J., D. Ruvolo, M. Jasielec, C. Xiong, E. Grant and J. C. Morris (2015). "Absence of practice effects in preclinical Alzheimer's disease." Neuropsychology 29(6): 940-948. Jack, C. R., Jr., D. S. Knopman, S. D. Weigand, H. J. Wiste, P. Vemuri, V. Lowe, K. Kantarci, J. L. Gunter, M. L. Senjem, R. J. Ivnik, R. O. Roberts, W. A. Rocca, B. F. Boeve and R. C. Petersen (2012). "An operational approach to National Institute on Aging-Alzheimer's Association criteria for preclinical Alzheimer disease." Ann Neurol 71(6): 765-775.

Jack, C. R., Jr., H. J. Wiste, S. D. Weigand, D. S. Knopman, M. M. Mielke, P. Vemuri, V. Lowe, M. L. Senjem, J. L. Gunter, D. Reyes, M. M. Machulda, R. Roberts and R. C. Petersen (2015). "Different definitions of neurodegeneration produce similar amyloid/neurodegeneration biomarker group findings." Brain 138(Pt 12): 3747-3759. Jack, C. R., Jr., H. J. Wiste, S. D. Weigand, W. A. Rocca, D. S. Knopman, M. M. Mielke, V. J. Lowe, M. L. Senjem, J. L. Gunter, G. M. Preboske, V. S. Pankratz, P. Vemuri and R. C. Petersen (2014). "Age-specific population frequencies of cerebral beta-amyloidosis and neurodegeneration among people with normal cognitive function aged 50-89 years: a crosssectional study." Lancet Neurol 13(10): 997-1005.

Jicha, G. A., E. L. Abner, F. A. Schmitt, R. J. Kryscio, K. P. Riley, G. E. Cooper, N. Stiles, M. S. Mendiondo, C. D. Smith, L. J. Van Eldik and P. T. Nelson (2012). "Preclinical AD Workgroup staging: pathological correlates and potential challenges." Neurobiol Aging 33(3): 622 e $621-622$ e616.

Ju, Y. E., J. S. McLeland, C. D. Toedebusch, C. Xiong, A. M. Fagan, S. P. Duntley, J. C. Morris and D. M. Holtzman (2013). "Sleep quality and preclinical Alzheimer disease." JAMA Neurol 70(5): 587-593.

Knopman, D. S., C. R. Jack, Jr., H. J. Wiste, S. D. Weigand, P. Vemuri, V. Lowe, K. Kantarci, J. L. Gunter, M. L. Senjem, R. J. Ivnik, R. O. Roberts, B. F. Boeve and R. C. Petersen (2012). "Short-term clinical outcomes for stages of NIA-AA preclinical Alzheimer disease." Neurology 78(20): 1576-1582.

Knopman, D. S., C. R. Jack, Jr., H. J. Wiste, S. D. Weigand, P. Vemuri, V. J. Lowe, K. Kantarci, J. L. Gunter, M. L. Senjem, M. M. Mielke, R. O. Roberts, B. F. Boeve and R. C. Petersen (2013). "Brain injury biomarkers are not dependent on beta-amyloid in normal elderly." Ann Neurol 73(4): 472-480.

Lim, Y. Y., P. Maruff, R. H. Pietrzak, D. Ames, K. A. Ellis, K. Harrington, N. T. Lautenschlager, C. Szoeke, R. N. Martins, C. L. Masters, V. L. Villemagne, C. C. Rowe and A. R. Group (2014). "Effect of amyloid on memory and non-memory decline from preclinical to clinical Alzheimer's disease." Brain 137(Pt 1): 221-231.

Lim, Y. Y., P. J. Snyder, R. H. Pietrzak, A. Ukiqi, V. L. Villemagne, D. Ames, O. Salvado, P. Bourgeat, R. N. Martins, C. L. Masters, C. C. Rowe and P. Maruff (2016). "Sensitivity of 
composite scores to amyloid burden in preclinical Alzheimer's disease: Introducing the Zscores of Attention, Verbal fluency, and Episodic memory for Nondemented older adults composite score." Alzheimers Dement (Amst) 2: 19-26.

Lim, Y. Y., V. L. Villemagne, S. M. Laws, D. Ames, R. H. Pietrzak, K. A. Ellis, K. D. Harrington, P. Bourgeat, O. Salvado, D. Darby, P. J. Snyder, A. I. Bush, R. N. Martins, C. L. Masters, C. C. Rowe, P. J. Nathan, P. Maruff, B. Australian Imaging and G. Lifestyle Research (2013). "BDNF Val66Met, Abeta amyloid, and cognitive decline in preclinical Alzheimer's disease." Neurobiol Aging 34(11): 2457-2464.

Mielke, M. M., H. J. Wiste, S. D. Weigand, D. S. Knopman, V. J. Lowe, R. O. Roberts, Y. E. Geda, D. M. Swenson-Dravis, B. F. Boeve, M. L. Senjem, P. Vemuri, R. C. Petersen and C. R. Jack, Jr. (2012). "Indicators of amyloid burden in a population-based study of cognitively normal elderly." Neurology 79(15): 1570-1577.

Mormino, E. C., R. A. Betensky, T. Hedden, A. P. Schultz, R. E. Amariglio, D. M. Rentz, K. A. Johnson and R. A. Sperling (2014). "Synergistic effect of beta-amyloid and neurodegeneration on cognitive decline in clinically normal individuals." JAMA Neurol 71(11): 1379-1385.

Mormino, E. C., R. A. Betensky, T. Hedden, A. P. Schultz, A. Ward, W. Huijbers, D. M. Rentz, K. A. Johnson, R. A. Sperling, I. Alzheimer's Disease Neuroimaging, B. Australian Imaging, A. Lifestyle Flagship Study of and S. Harvard Aging Brain (2014). "Amyloid and APOE epsilon4 interact to influence short-term decline in preclinical Alzheimer disease." Neurology 82(20): 1760-1767.

Morris, J. C., C. M. Roe, E. A. Grant, D. Head, M. Storandt, A. M. Goate, A. M. Fagan, D. M. Holtzman and M. A. Mintun (2009). "Pittsburgh Compound B imaging and prediction of progression from cognitive normality to symptomatic Alzheimer disease." Archives of Neurology 66(12): 1469-1475.

Morris, J. C., C. M. Roe, C. Xiong, A. M. Fagan, A. M. Goate, D. M. Holtzman and M. A. Mintun (2010). "APOE predicts amyloid-beta but not tau Alzheimer pathology in cognitively normal aging." Ann Neurol 67(1): 122-131.

Nation, D. A., E. C. Edmonds, K. J. Bangen, L. Delano-Wood, B. K. Scanlon, S. D. Han, S. D. Edland, D. P. Salmon, D. R. Galasko, M. W. Bondi and I. Alzheimer's Disease Neuroimaging Initiative (2015). "Pulse pressure in relation to tau-mediated neurodegeneration, cerebral amyloidosis, and progression to dementia in very old adults." JAMA Neurol 72(5): 546-553. Papp, K. V., R. E. Amariglio, E. C. Mormino, T. Hedden, M. Dekhytar, K. A. Johnson, R. A. Sperling and D. M. Rentz (2015). "Free and cued memory in relation to biomarker-defined abnormalities in clinically normal older adults and those at risk for Alzheimer's disease." Neuropsychologia 73: 169-175.

Papp, K. V., E. C. Mormino, R. E. Amariglio, C. Munro, A. Dagley, A. P. Schultz, K. A. Johnson, R. A. Sperling and D. M. Rentz (2016). "Biomarker validation of a decline in semantic processing in preclinical Alzheimer's disease." Neuropsychology 30(5): 624-630. Pascoal, T. A., S. Mathotaarachchi, S. Mohades, A. L. Benedet, C. O. Chung, M. Shin, S. Wang, T. Beaudry, M. S. Kang, J. P. Soucy, A. Labbe, S. Gauthier and P. Rosa-Neto (2016). "Amyloid-beta and hyperphosphorylated tau synergy drives metabolic decline in preclinical Alzheimer's disease." Mol Psychiatry.

Pettigrew, C., A. Soldan, A. Moghekar, M. C. Wang, A. L. Gross, R. O'Brien and M. Albert (2015). "Relationship between cerebrospinal fluid biomarkers of Alzheimer's disease and cognition in cognitively normal older adults." Neuropsychologia 78: 63-72.

Pietrzak, R. H., Y. Y. Lim, D. Ames, K. Harrington, C. Restrepo, R. N. Martins, A. Rembach, S. M. Laws, C. L. Masters, V. L. Villemagne, C. C. Rowe, P. Maruff, B. Australian Imaging and G. Lifestyle Research (2015). "Trajectories of memory decline in preclinical Alzheimer's 
disease: results from the Australian Imaging, Biomarkers and Lifestyle Flagship Study of ageing." Neurobiol Aging 36(3): 1231-1238.

Pietrzak, R. H., Y. Y. Lim, A. Neumeister, D. Ames, K. A. Ellis, K. Harrington, N. T. Lautenschlager, C. Restrepo, R. N. Martins, C. L. Masters, V. L. Villemagne, C. C. Rowe, P. Maruff, B. Australian Imaging and G. Lifestyle Research (2015). "Amyloid-beta, anxiety, and cognitive decline in preclinical Alzheimer disease: a multicenter, prospective cohort study." JAMA Psychiatry 72(3): 284-291.

Pike, K. E., K. A. Ellis, V. L. Villemagne, N. Good, G. Chetelat, D. Ames, C. Szoeke, S. M. Laws, G. Verdile, R. N. Martins, C. L. Masters and C. C. Rowe (2011). "Cognition and betaamyloid in preclinical Alzheimer's disease: data from the AIBL study." Neuropsychologia 49(9): 2384-2390.

Racine, A. M., N. Adluru, A. L. Alexander, B. T. Christian, O. C. Okonkwo, J. Oh, C. A. Cleary, A. Birdsill, A. T. Hillmer, D. Murali, T. E. Barnhart, C. L. Gallagher, C. M. Carlsson, H. A. Rowley, N. M. Dowling, S. Asthana, M. A. Sager, B. B. Bendlin and S. C. Johnson (2014). "Associations between white matter microstructure and amyloid burden in preclinical Alzheimer's disease: A multimodal imaging investigation." Neuroimage Clin 4: 604-614. Racine, A. M., R. L. Koscik, S. E. Berman, C. R. Nicholas, L. R. Clark, O. C. Okonkwo, H. A. Rowley, S. Asthana, B. B. Bendlin, K. Blennow, H. Zetterberg, C. E. Gleason, C. M. Carlsson and S. C. Johnson (2016). "Biomarker clusters are differentially associated with longitudinal cognitive decline in late midlife." Brain.

Riley, K. P., G. A. Jicha, D. Davis, E. L. Abner, G. E. Cooper, N. Stiles, C. D. Smith, R. J. Kryscio, P. T. Nelson, L. J. Van Eldik and F. A. Schmitt (2011). "Prediction of preclinical Alzheimer's disease: longitudinal rates of change in cognition." J Alzheimers Dis 25(4): 707717.

(73Soldan, A., C. Pettigrew, Q. Cai, M. C. Wang, A. R. Moghekar, R. J. O'Brien, O. A. Selnes, M. S. Albert and B. R. Team (2016). "Hypothetical Preclinical Alzheimer Disease Groups and Longitudinal Cognitive Change." JAMA Neurol 73(6): 698-705.

Stark, S. L., C. M. Roe, E. A. Grant, H. Hollingsworth, T. L. Benzinger, A. M. Fagan, V. D. Buckles and J. C. Morris (2013). "Preclinical Alzheimer disease and risk of falls." Neurology 81(5): 437-443.

Sutphen, C. L., M. S. Jasielec, A. R. Shah, E. M. Macy, C. Xiong, A. G. Vlassenko, T. L. Benzinger, E. E. Stoops, H. M. Vanderstichele, B. Brix, H. D. Darby, M. L. Vandijck, J. H. Ladenson, J. C. Morris, D. M. Holtzman and A. M. Fagan (2015). "Longitudinal Cerebrospinal Fluid Biomarker Changes in Preclinical Alzheimer Disease During Middle Age." JAMA Neurol 72(9): 1029-1042.

Thai, C., Y. Y. Lim, V. L. Villemagne, S. M. Laws, D. Ames, K. A. Ellis, S. R. Rainey-Smith, R. N. Martins, C. L. Masters, C. C. Rowe, P. Maruff, B. Australian Imaging and G. Lifestyle Research (2015). "Amyloid-Related Memory Decline in Preclinical Alzheimer's Disease Is Dependent on APOE epsilon4 and Is Detectable over 18-Months." PLoS One 10(10): $\mathrm{e} 0139082$.

Thal, D. R., C. von Arnim, W. S. Griffin, H. Yamaguchi, R. E. Mrak, J. Attems and A. R. Upadhaya (2013). "Pathology of clinical and preclinical Alzheimer's disease." Eur Arch Psychiatry Clin Neurosci 263 Suppl 2: S137-145.

Valech, N., M. A. Mollica, J. Olives, A. Tort, J. Fortea, A. Lleo, S. S. Belen, J. L. Molinuevo and L. Rami (2015). "Informants' Perception of Subjective Cognitive Decline Helps to Discriminate Preclinical Alzheimer's Disease from Normal Aging." J Alzheimers Dis 48 Suppl 1: S87-98.

van Harten, A. C., L. L. Smits, C. E. Teunissen, P. J. Visser, T. Koene, M. A. Blankenstein, P. Scheltens and W. M. van der Flier (2013). "Preclinical AD predicts decline in memory and executive functions in subjective complaints." Neurology 81(16): 1409-1416. 
Voevodskaya, O., P. C. Sundgren, O. Strandberg, H. Zetterberg, L. Minthon, K. Blennow, L. O. Wahlund, E. Westman, O. Hansson and F. s. g. Swedish Bio (2016). "Myo-inositol changes precede amyloid pathology and relate to APOE genotype in Alzheimer disease." Neurology 86(19): 1754-1761.

Vos, S. J., B. A. Gordon, Y. Su, P. J. Visser, D. M. Holtzman, J. C. Morris, A. M. Fagan and T. L. Benzinger (2016). "NIA-AA staging of preclinical Alzheimer disease: discordance and concordance of CSF and imaging biomarkers." Neurobiol Aging 44: 1-8.

Vos, S. J., C. Xiong, P. J. Visser, M. S. Jasielec, J. Hassenstab, E. A. Grant, N. J. Cairns, J. C. Morris, D. M. Holtzman and A. M. Fagan (2013). "Preclinical Alzheimer's disease and its outcome: a longitudinal cohort study." Lancet Neurol 12(10): 957-965.

Wang, L., T. L. Benzinger, J. Hassenstab, T. Blazey, C. Owen, J. Liu, A. M. Fagan, J. C.

Morris and B. M. Ances (2015). "Spatially distinct atrophy is linked to beta-amyloid and tau in preclinical Alzheimer disease." Neurology 84(12): 1254-1260.

Whitwell, J. L., N. Tosakulwong, S. D. Weigand, M. L. Senjem, V. J. Lowe, J. L. Gunter, B. F. Boeve, D. S. Knopman, B. C. Dickerson, R. C. Petersen and C. R. Jack, Jr. (2013). "Does amyloid deposition produce a specific atrophic signature in cognitively normal subjects?" Neuroimage Clin 2: 249-257. 
TABLE 2. STUDIES METHODOLOGY

\begin{tabular}{|c|c|c|c|c|c|}
\hline $\begin{array}{l}\text { Author and } \\
\text { year of } \\
\text { publication }\end{array}$ & $\begin{array}{l}\text { Follow-up } \\
\text { duration } \\
\text { (months) }\end{array}$ & $\begin{array}{c}\text { normal } \\
\text { cognition }\end{array}$ & $\begin{array}{c}\text { Cognitive } \\
\text { decline/outcome }\end{array}$ & $\begin{array}{l}\text { AD } \\
\text { pathophysiologi } \\
\text { cal signature }\end{array}$ & $\begin{array}{l}\text { Definition of } \\
\text { biomarker }\end{array}$ \\
\hline \multicolumn{6}{|c|}{ CROSS-SECTIONAL STUDIES } \\
\hline $\begin{array}{l}\text { (Morris, Roe } \\
\text { et al. 2010) }\end{array}$ & - & CDR & - & $\begin{array}{l}\text { PET-PIB and } \\
\text { CSF }\end{array}$ & $\begin{array}{c}\text { PET-Aß DEFINITION 1: PIB } \\
\text { MCBP } \geq 0.18 \\
\text { CSF DEFINITION } 1 \\
\text { CSF A } \beta 42 \text { levels below } 500 \\
\mathrm{pg} / \mathrm{mL}, \text { tau levels above } 500 \\
\mathrm{pg} / \mathrm{mL}, \text { and Ptau } 181 \text { levels } \\
\text { above } 80 \mathrm{pg} / \mathrm{mL} \\
\text { CSF biomarkers assay Innotest } \\
\text { ELISA }\end{array}$ \\
\hline $\begin{array}{l}\text { (Pike, Ellis } \\
\text { et al. 2011) }\end{array}$ & - & Cognitive battery & - & PET-PIB & $\begin{array}{c}\text { PET-Aß DEFINITION } 2 \\
\text { PIB SUVR } \geq 1.5\end{array}$ \\
\hline $\begin{array}{l}\text { (Mielke, } \\
\text { Wiste et al. } \\
\text { 2012) }\end{array}$ & - & Cognitive battery & - & PET-PIB & $\begin{array}{l}\text { PET-Aß DEFINITION } 3 \\
\text { PIB SUVR > } 1.4 \text { and } 1.5\end{array}$ \\
\hline $\begin{array}{l}\text { (Amariglio, } \\
\text { Becker et al. } \\
\text { 2012) }\end{array}$ & - & $\begin{array}{l}\text { CDR, MMSE, } \\
\text { GDS }\end{array}$ & - & PET-PIB & $\begin{array}{l}\text { PET-Aß DEFINITION } 4 \\
\text { PIB DVR } \geq 1.25\end{array}$ \\
\hline $\begin{array}{c}\text { (Jack, } \\
\text { Knopman et } \\
\text { al. 2012) }\end{array}$ & - & Cognitive battery & - & PET-PIB & $\begin{array}{l}\text { PET-Aß DEFINITION } 2 \\
\text { PIB SUVR } \geq 1.5\end{array}$ \\
\hline $\begin{array}{l}\text { (Harrington, } \\
\text { Chiang et al. } \\
\text { 2013) }\end{array}$ & - & $\begin{array}{c}\text { CDR and } \\
\text { cognitive battery }\end{array}$ & - & CSF A $3-42$ & $\begin{array}{c}\text { CSF DEFINITION } 2 \\
\text { Aß42/Tau ratio cutoff calculated } \\
\text { as } 2.7132 \\
\text { CSF biomarkers assay Innotest } \\
\text { ELISA }\end{array}$ \\
\hline $\begin{array}{l}\text { (Whitwell, } \\
\text { Tosakulwon } \\
\text { g et al. 2013) }\end{array}$ & - & $\begin{array}{c}\text { CDR and } \\
\text { Cognitive battery }\end{array}$ & - & PET-PIB & $\begin{array}{c}\text { PET-Aß DEFINITION } 2 \\
\text { PIB SUVR } \geq 1.5\end{array}$ \\
\hline $\begin{array}{l}(\mathrm{Ju}, \\
\text { McLeland et } \\
\text { al. 2013) }\end{array}$ & - & CDR & - & CSF A $3-42$ & $\begin{array}{c}\text { CSF DEFINITION } 3 \\
\text { A beta } 42<500 \mathrm{nmol} / \mathrm{mL} \\
\text { CSF biomarkers assay Innotest } \\
\text { ELISA }\end{array}$ \\
\hline $\begin{array}{l}\text { (Knopman, } \\
\text { Jack et al. } \\
\text { 2013) }\end{array}$ & - & $\begin{array}{c}\text { CDR and } \\
\text { Cognitive battery }\end{array}$ & - & PET-PIB & $\begin{array}{c}\text { PET-Aß DEFINITION } 2 \\
\text { PiB SUVR } \geq 1.5\end{array}$ \\
\hline $\begin{array}{l}\text { (Brier, } \\
\text { Thomas et } \\
\text { al. 2014) }\end{array}$ & - & NA & - & CSF $A ß$ or Tau & $\begin{array}{c}\text { CSF DEFINITION } 4 \\
\text { A } \beta 42<500 \mathrm{pg} / \mathrm{mL} \\
\text { tau }>440 \mathrm{pg} / \mathrm{mL} \\
\text { NIA AA preclinical AD staging } \\
\text { CSF biomarkers assay Innotest } \\
\text { ELISA } \\
\end{array}$ \\
\hline $\begin{array}{l}\text { (Brier, } \\
\text { Thomas et } \\
\text { al. 2014) }\end{array}$ & - & NA & - & CSF $A ß$ or Tau & $\begin{array}{c}\text { CSF DEFINITION } 4 \\
\text { A } \beta 42<500 \mathrm{pg} / \mathrm{mL} \\
\text { tau }>440 \mathrm{pg} / \mathrm{mL} \\
\text { NIA AA preclinical AD staging } \\
\text { CSF biomarkers assay Innotest }\end{array}$ \\
\hline
\end{tabular}




\begin{tabular}{|c|c|c|c|c|c|}
\hline & & & & & ELISA \\
\hline $\begin{array}{l}\text { (Jack, Wiste } \\
\text { et al. 2014) }\end{array}$ & - & Cognitive battery & - & PET-PIB & $\begin{array}{l}\text { PET-Aß DEFINITION } 5 \\
\text { PiB SUVR } \geq 1.4\end{array}$ \\
\hline $\begin{array}{l}\text { (Racine, } \\
\text { Adluru et al. } \\
\text { 2014) }\end{array}$ & - & Cognitive battery & - & PET-PIB & $\begin{array}{l}\text { PET-Aß DEFINITION } 6 \\
\text { Visual assessment with } \\
\text { classification as amyloid } \\
\text { negative, positive or } \\
\text { indeterminate }\end{array}$ \\
\hline $\begin{array}{l}\text { (Fortea, } \\
\text { Vilaplana et } \\
\text { al. 2014) }\end{array}$ & - & MMSE & - & $\begin{array}{c}\text { CSF A } A \text { or P- } \\
\text { Tau }\end{array}$ & $\begin{array}{c}\text { CSF DEFINITION } 5 \\
\text { p-tau181p levels }>23 \mathrm{pg} / \mathrm{ml} \\
\text { A } \beta 1-42 \text { levels }<192 \mathrm{pg} / \mathrm{ml} \\
\text { CSF biomarkers assay Luminex }\end{array}$ \\
\hline $\begin{array}{l}\text { (Wang, } \\
\text { Benzinger et } \\
\text { al. 2015) }\end{array}$ & - & CDR & - & $\begin{array}{c}\text { CSF A } 3 \text { and } \\
\text { Tau }\end{array}$ & $\begin{array}{c}\text { CSF DEFINITION } 6 \\
\text { CSF A } 342 \leq 500 \mathrm{pg} / \mathrm{mL} \text { and } \\
\text { CSF tau } \geq 500 \mathrm{pg} / \mathrm{mL}, \\
\text { respectively, and negative if } \\
\text { CSF A } 342>500 \mathrm{pg} / \mathrm{mL} \text { and } \\
\text { CSF tau }<500 \mathrm{ng} / \mathrm{mL} \\
\text { CSF biomarkers assay Innotest } \\
\text { ELISA }\end{array}$ \\
\hline $\begin{array}{l}\text { (Doherty, } \\
\text { Schultz et al. } \\
\text { 2015) }\end{array}$ & - & Cognitive battery & - & PET-PIB & $\begin{array}{c}\text { PET-Aß DEFINITION } 7 \\
\text { Qualitative visual assessment }\end{array}$ \\
\hline $\begin{array}{l}\text { (Valech, } \\
\text { Mollica et al. } \\
\text { 2015) }\end{array}$ & - & CDR and MMSE & - & CSF A $\beta 42$ & $\begin{array}{l}\text { CSF DEFINITION } 7 \\
\text { CSF A } \beta 42 \leq 550 \mathrm{pg} / \mathrm{ml}\end{array}$ \\
\hline $\begin{array}{l}\text { (Jack, Wiste } \\
\text { et al. 2015) }\end{array}$ & - & Multiple tests & - & PET-PIB & $\begin{array}{c}\text { PET-A } ß \text { DEFINITION } 5 \\
\text { SUVR }>1.4\end{array}$ \\
\hline $\begin{array}{l}\text { (Alcolea, } \\
\text { Martinez- } \\
\text { Lage et al. } \\
\text { 2015) }\end{array}$ & - & $\begin{array}{l}\text { MMSE, FCSRT } \\
\text { and cognitive } \\
\text { battery }\end{array}$ & - & $\mathrm{CSF}$ & $\begin{array}{c}\text { CSF DEFINITION } 8 \\
\text { stage } 0(\mathrm{~A} \beta 42 \geq 550 \mathrm{pg} / \mathrm{mL}, \mathrm{t}- \\
\text { tau } \leq 350 \mathrm{pg} / \mathrm{mL}, \\
\text { and } \mathrm{p} \text {-tau } \leq 61 \mathrm{pg} / \mathrm{mL}), \text { stage } 1 \\
(\mathrm{~A} \beta 42 \geq 550 \mathrm{pg} / \mathrm{mL}, \mathrm{t} \text {-tau } \leq 350 \\
\mathrm{pg} / \mathrm{mL}, \text { and } \mathrm{p}-\mathrm{tau} \leq 61 \mathrm{pg} / \mathrm{mL}), \\
\text { stage } 2(\mathrm{~A} \beta 42 \leq 550 \mathrm{pg} / \mathrm{mL} \text { and } \\
\text { either t-tau } \geq 350 \mathrm{pg} / \mathrm{mL} \text { or } \mathrm{p}- \\
\text { tau } \geq 61 \mathrm{pg} / \mathrm{mL}), \text { or stage } 3 \\
\text { (stage } 2 \\
\text { plus subtle cognitive decline, } \\
\text { defined as an episodic memory } \\
\text { composite } \\
\text { score in the lowest } 10 \text { th } \\
\text { percentile). } \\
\text { CSF biomarkers assay Innotest } \\
\text { ELISA }\end{array}$ \\
\hline $\begin{array}{l}\text { (Papp, } \\
\text { Amariglio et } \\
\text { al. 2015) }\end{array}$ & - & $\begin{array}{l}\text { CDR, MMSE, } \\
\text { GDS, Delayed } \\
\text { logical memory } \\
\text { recall }\end{array}$ & - & PET-PIB & $\begin{array}{c}\text { PET-Aß DEFINITION } 8 \\
\text { PIB data were analyzed as } \\
\text { standard uptake value ratios } \\
\text { (SUVR), and a Gaussian } \\
\text { mixture modeling approach was } \\
\text { used to classify HABS CNs as } \\
\text { A } \beta+\text { or A } \beta \text { - (cut-off }\end{array}$ \\
\hline
\end{tabular}




\begin{tabular}{|c|c|c|c|c|c|}
\hline & & & & & value $=1.20)$ \\
\hline $\begin{array}{c}\{\text { Hassenstab, } \\
2016 \\
\# 23381\}\end{array}$ & - & CDR & - & $\begin{array}{l}\text { CSF and PET- } \\
\text { PIB (+ } \\
\text { hippocampal } \\
\text { volume) }\end{array}$ & $\begin{array}{c}\text { PET-Aß DEFINITION } 9 \\
\text { PET: mean cortical binding } \\
\text { potential }>0.2245 ; \\
\text { CSF DEFINITION } 9 \\
\text { CSF: Ab42<459 pg/ml, tau }>339 \\
\text { pg/ml }\end{array}$ \\
\hline $\begin{array}{l}\text { (Voevodskay } \\
\text { a, Sundgren } \\
\text { et al. 2016) }\end{array}$ & - & MMSE & - & $\mathrm{CSF}$ & $\begin{array}{l}\text { CSF DEFINITION } 10 \\
\text { CSF : Aß42 } \leq 530 \mathrm{ng} / \mathrm{L}\end{array}$ \\
\hline
\end{tabular}

LONGITUDINAL STUDIES

\begin{tabular}{|c|c|c|c|c|c|}
\hline $\begin{array}{l}\text { (Morris, Roe } \\
\text { et al. 2009) }\end{array}$ & 28.8 & CDR & CDR change & PET-PIB & Correlation study (no cut off) \\
\hline $\begin{array}{l}\text { (Craig- } \\
\text { Schapiro, } \\
\text { Perrin et al. } \\
\text { 2010) }\end{array}$ & - & CDR & CDR change & CSF & Correlation study (no cut off) \\
\hline $\begin{array}{l}\text { (Desikan, } \\
\text { McEvoy et } \\
\text { al. 2012) }\end{array}$ & $6-42$ & CDR & MRI & $\mathrm{CSF}$ & $\begin{array}{c}\text { CSF DEFINITION } 5 \\
\text { p-tau181p levels }>23 \mathrm{pg} / \mathrm{ml} \text {; } \\
\text { A } \beta 1-42 \text { levels }<192 \mathrm{pg} / \mathrm{ml} \\
\text { CSF biomarkers assay Luminex }\end{array}$ \\
\hline $\begin{array}{l}\text { (Knopman, } \\
\text { Jack et al. } \\
\text { 2012) }\end{array}$ & 12 & Cognitive battery & Cognitive battery & PET-PIB & $\begin{array}{c}\text { PET-Aß DEFINITION } 2 \\
\text { PIB SUVR } \geq 1.5\end{array}$ \\
\hline $\begin{array}{l}\text { (van Harten, } \\
\text { Smits et al. } \\
\text { 2013) }\end{array}$ & 24 & Cognitive battery & Cognitive battery & $\begin{array}{c}\text { CSF Aß/ Tau or } \\
\text { P-Tau }\end{array}$ & $\begin{array}{c}\text { CSF DEFINITION } 11 \\
550 \mathrm{ng} / \mathrm{L} \text { for A } 342,, 375 \mathrm{ng} / \mathrm{L} \\
\text { for Tau, and ,52 ng/L for pTau } \\
\text { CSF biomarkers assay Innotest } \\
\text { ELISA } \\
\end{array}$ \\
\hline $\begin{array}{l}\text { (Vos, Xiong } \\
\text { et al. 2013) }\end{array}$ & 60 & CDR & CDR & $\begin{array}{c}\text { CSF Aß/ Tau or } \\
\text { P-Tau }\end{array}$ & $\begin{array}{c}\text { CSF DEFINITION } 12 \\
<459 \mathrm{ng} / \mathrm{L} \text { for } \mathrm{A} \beta 1-42,>339 \\
\mathrm{ng} / \mathrm{L} \text { for t-tau, and }>67 \mathrm{ng} / \mathrm{L} \text { for } \\
\text { p-tau } \\
\text { CSF biomarkers assay Innotest } \\
\text { ELISA } \\
\end{array}$ \\
\hline $\begin{array}{l}\text { (Stark, Roe } \\
\text { et al. 2013) }\end{array}$ & 12 & CDR & CDR & PET-PIB & $\begin{array}{c}\text { PET-Aß DEFINITION } 10 \\
\text { PIB MCBP } \\
\text { High and low groups were } \\
\text { constructed for all } 4 \text { biomarkers } \\
\text { and the } 2 \text { ratios with the most } \\
\text { extreme quartile were assigned } \\
\text { as high for that variable }\end{array}$ \\
\hline $\begin{array}{c}\text { (Lim, } \\
\text { Villemagne } \\
\text { et al. 2013) } \\
\end{array}$ & 36 & Cognitive batttery & 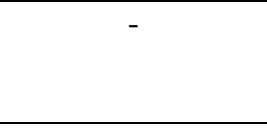 & PET-PIB & $\begin{array}{c}\text { PET-Aß DEFINITION } 2 \\
\text { PIB SUVR } \geq 1.5\end{array}$ \\
\hline $\begin{array}{l}\text { (Lim, Maruff } \\
\text { et al. 2014) }\end{array}$ & 36 & Cognitive batttery & Cognitive battery & $\begin{array}{c}\text { PET-PIB or } \\
\text { PET florbetapir } \\
\text { or } \\
\text { PET } \\
\text { Flutemetamol }\end{array}$ & $\begin{array}{c}\text { PET-Aß DEFINITION } 11 \\
\text { PIB SUVR } \geq 1.9 \\
\text { Flutemetamol SUVR } \geq 2.19 \\
\text { Florbetapir } S U V R \geq 1.29\end{array}$ \\
\hline
\end{tabular}




\begin{tabular}{|c|c|c|c|c|c|}
\hline $\begin{array}{l}\text { (Mormino, } \\
\text { Betensky et } \\
\text { al. 2014) }\end{array}$ & 24 & $\begin{array}{c}\text { CDR and } \\
\text { cognitive batttery }\end{array}$ & Cognitive battery & PET-PIB & $\begin{array}{c}\text { PET-Aß DEFINITION } 12 \\
\text { PIB SUVR } \\
\text { Gaussian Mixture Model: } \\
\text { individuals with greater than } \\
50 \% \text { probability of belonging to } \\
\text { their cohort's high Aß } \\
\text { distribution were labeled high } \\
\text { Aß }\end{array}$ \\
\hline $\begin{array}{l}\text { (Donohue, } \\
\text { Sperling et } \\
\text { al. 2014) }\end{array}$ & 36 & CDR & $\begin{array}{l}\text { Composite score } \\
\text { (ADCS-PACC) }\end{array}$ & $\begin{array}{l}\text { PET-PIB or } \\
\text { CSF A } 42\end{array}$ & $\begin{array}{c}\text { PET-Aß DEFINITION } 2 \\
\text { PiB SUVR }>1.5 \\
\text { CSF DEFINITION } 5 \\
\text { Ab42 }<192 \mathrm{pg} / \mathrm{ml} \\
\text { CSF biomarkers assay Luminex }\end{array}$ \\
\hline $\begin{array}{l}\text { (Ayutyanont, } \\
\text { Langbaum et } \\
\text { al. 2014) }\end{array}$ & $24-60$ & Clinical & Cognitive battery & $\begin{array}{c}\text { E280A PSen } 1 \\
\text { mutation }\end{array}$ & $\begin{array}{c}\text { GENETIC DEFINITION } 1 \\
\text { E280A PSen } 1 \text { mutation }\end{array}$ \\
\hline $\begin{array}{l}\text { (Mormino, } \\
\text { Betensky et } \\
\text { al. 2014) }\end{array}$ & 18 & CDR, MMSE & $\begin{array}{l}\text { MMSE and Logical } \\
\text { Memory I and IIa }\end{array}$ & $\begin{array}{l}\text { PET-PIB or } \\
\text { Florbetapir }\end{array}$ & $\begin{array}{c}\text { PET-Aß DEFINITION } 12 \\
\text { PIB or florbetapir SUVR } \\
\text { Gaussian Mixture Model: } \\
\text { individuals with greater than } \\
50 \% \text { probability of belonging to } \\
\text { their cohort's high Aß } \\
\text { distribution were labeled high } \\
\text { Aß }\end{array}$ \\
\hline $\begin{array}{l}\text { (Pietrzak, } \\
\text { Lim et al. } \\
\text { 2015) }\end{array}$ & 54 & $\begin{array}{c}\text { Clinical } \\
\text { consensus }\end{array}$ & Composite score & $\begin{array}{l}\text { PET-PIB or } \\
\text { PET florbetapir } \\
\text { or PET } \\
\text { Flutemetamol }\end{array}$ & $\begin{array}{c}\text { PET-Aß DEFINITION } 13 \\
\text { PIB SUVR } \geq 1.5 \\
\text { florbetapir } S U V R \geq 1.1 \\
\text { flutemetamol SUVR } \geq 0.62 \\
\text { (pons as reference region) }\end{array}$ \\
\hline $\begin{array}{l}\text { (Pietrzak, } \\
\text { Lim et al. } \\
\text { 2015) }\end{array}$ & 54 & $\begin{array}{c}\text { Clinical } \\
\text { consensus }\end{array}$ & Composite score & $\begin{array}{l}\text { PET-PIB or } \\
\text { PET florbetapir } \\
\text { or PET } \\
\text { flutemetamol }\end{array}$ & $\begin{array}{c}\text { PET-Aß DEFINITION } 13 \\
\text { PIB SUVR } \geq 1.5 \\
\text { florbetapir SUVR } \geq 1.1 \\
\text { flutemetamol SUVR } \geq 0.62 \\
\text { (pons as reference region) }\end{array}$ \\
\hline $\begin{array}{l}\text { (Nation, } \\
\text { Edmonds et } \\
\text { al. 2015) }\end{array}$ & 28 & - & $\begin{array}{l}\text { ADNI criteria for } \\
\text { MCI or dementia } \\
\text { diagnosis }\end{array}$ & $\begin{array}{l}\text { CSF Aß or P- } \\
\text { Tau }\end{array}$ & $\begin{array}{c}\text { CSF DEFINITION } 5 \\
\text { P-tau levels }>23 \mathrm{pg} / \mathrm{ml} ; \text { T-tau } \\
(\geq 93 \mathrm{pg} / \mathrm{mL} ; \mathrm{A} \beta 1-42 \\
\text { levels }<192 \mathrm{pg} / \mathrm{ml} \\
\text { CSF biomarkers assay Luminex }\end{array}$ \\
\hline $\begin{array}{l}\text { (Thai, Lim et } \\
\text { al. 2015) }\end{array}$ & 18 & - & $\begin{array}{l}\text { Cogsate Brief } \\
\text { Battery, CVLT-II }\end{array}$ & PET amyloid & $\begin{array}{c}\text { PET-Aß DEFINITION } 13 \\
\text { several isotopes. SUVR } \geq 1.5 \text { for } \\
11 \mathrm{C}-\mathrm{PiB}, \mathrm{SUVR} \geq 1.1 \text { for } 18 \mathrm{~F}- \\
\text { florbetapir, and } \mathrm{SUVR} \geq 0.62 \text { for } \\
18 \mathrm{~F}-\text { flutemetamol }\end{array}$ \\
\hline $\begin{array}{l}\text { (Pettigrew, } \\
\text { Soldan et al. } \\
\text { 2015) }\end{array}$ & 142 & Cognitive battery & Cognitive battery & $\begin{array}{l}\text { (study of } \\
\text { correlation } \\
\text { between CSF } \\
\text { tau and } \\
\text { cognition) }\end{array}$ & Correlation study (no cut off) \\
\hline $\begin{array}{l}\text { (Sutphen, } \\
\text { Jasielec et al. } \\
\text { 2015) }\end{array}$ & 72 & CDR & CDR & $\begin{array}{l}\text { CSF CSF Aß } \\
\text { (PET-PIB in a } \\
\text { subpopulation) }\end{array}$ & $\begin{array}{c}\text { CSF DEFINITION } 13 \\
\text { CSF A } \beta 42 \text { cutoff : } 1041 \mathrm{pg} / \mathrm{mL} \\
\text { (INNOTEST kit) }\end{array}$ \\
\hline $\begin{array}{l}\text { (Papp, } \\
\text { Mormino et } \\
\text { al. 2016) }\end{array}$ & 19 & MMSE & $\begin{array}{l}\text { Letter and category } \\
\text { fluencies }\end{array}$ & PET-PIB & $\begin{array}{c}\text { PET-Aß DEFINITION } 14 \\
\text { Gaussian mixture modeling } \\
\text { approach } \\
\text { SUVR }>1.2\end{array}$ \\
\hline
\end{tabular}




\begin{tabular}{|c|c|c|c|c|c|}
\hline $\begin{array}{l}\text { (Soldan, } \\
\text { Pettigrew et } \\
\text { al. 2016) }\end{array}$ & 132 & Cognitive battery & Cognitive battery & $\begin{array}{l}\text { CSF } \beta \text {-amyloid, } \\
\text { tau or p-tau }\end{array}$ & $\begin{array}{c}\text { CSF DEFINITION } 14 \\
\text { CSF A } \beta 1-42 \text { levels in the } \\
\text { lower one-third of the } \\
\text { distribution of participants } \\
(<374.5 \mathrm{pg} / \mathrm{mL}) \text { or having tau } \\
(>74.9 \mathrm{pg} / \mathrm{mL}) \text { or } \mathrm{p}-\mathrm{tau}(>39.4 \\
\mathrm{pg} / \mathrm{mL}) \text { levels in the upper } \\
\text { one-third of the distribution. }\end{array}$ \\
\hline $\begin{array}{l}\text { (Racine, } \\
\text { Koscik et al. } \\
\text { 2016) }\end{array}$ & 72 & Cognitive battery & Cognitive battery & $\begin{array}{l}\text { CSF } ß \text {-amyloid } \\
\text { and tau }\end{array}$ & $\begin{array}{l}\text { Used to identify clusters, no } \\
\text { predefined threshold }\end{array}$ \\
\hline $\begin{array}{l}\text { (Pascoal, } \\
\text { Mathotaarac } \\
\text { hchi et al. } \\
\text { 2016) }\end{array}$ & 24 & CDR and MMSE & - & $\begin{array}{l}\text { CSF ß-amyloid } \\
\text { and tau, } \\
\text { Florbetapir PET } \\
\text { SUVR }\end{array}$ & Correlation study (no cut off) \\
\hline $\begin{array}{l}\text { (Vos, } \\
\text { Gordon et al. } \\
\text { 2016) }\end{array}$ & 39.6 & $\begin{array}{l}\text { CDR, MMSE, } \\
\text { cognitive battery }\end{array}$ & $\begin{array}{l}\text { CDR, MMSE, } \\
\text { cognitive battery }\end{array}$ & $\begin{array}{l}\text { CSF } ß \text {-amyloid } \\
\text { and tau, PIB- } \\
\text { PET }\end{array}$ & $\begin{array}{c}\text { PET-Aß DEFINITION } 9 \\
\text { PET PIB MCBP > 0.2245; } \\
\text { CSF DEFINITION } 9 \\
\text { CSF }: \text { Ab42<459 pg/ml, } \\
\text { tau }>\mathbf{3 3 9} \mathbf{~ p g} / \mathbf{m l} \\
(\text { CSF definition } 9) \\
\end{array}$ \\
\hline $\begin{array}{l}\text { (Bilgel, } \\
\text { Prince et al. } \\
\text { 2016) }\end{array}$ & 39.6 & $\begin{array}{l}\text { Clinical MCI and } \\
\text { dementia status }\end{array}$ & $\begin{array}{l}\text { Clinical MCI and } \\
\text { dementia status }\end{array}$ & PET-PIB & $\begin{array}{l}\text { PET-Aß DEFINITION } 15 \\
\text { mean cortical } \\
\text { DVR threshold of } 1.06\end{array}$ \\
\hline $\begin{array}{l}\text { (Brier, } \\
\text { McCarthy et } \\
\text { al. 2016) }\end{array}$ & 39.5 & CDR & CDR & PET-PIB & $\begin{array}{c}\text { PET-Aß DEFINITION } 16 \\
\text { MC SUVR across the } \\
\text { precuneus, } \\
\text { prefrontal, gyrus rectus, and } \\
\text { temporal FreeSurfer regions of } \\
\text { interest (ROIs) }>1.42 \\
\text { (equivalent to MCBP values for } \\
\text { PIB at or above } 0.18 \text { ) }\end{array}$ \\
\hline $\begin{array}{l}\text { (Clark, } \\
\text { Racine et al. } \\
\text { 2016) }\end{array}$ & 92.4 & Cognitive battery & $\begin{array}{c}\text { Cognitive } \\
\text { Composite scores }\end{array}$ & PET-PIB & $\begin{array}{l}\text { No cut off for PET-PIB (studied } \\
\text { across groups of subjects who } \\
\text { stay cognitively normal or } \\
\text { become MCI) }\end{array}$ \\
\hline $\begin{array}{l}\text { (Harrington, } \\
\text { Gould et al. } \\
\text { 2016) }\end{array}$ & 54 & Cognitive batttery & $\begin{array}{c}\mathrm{MCI} / \mathrm{AD} \\
\text { progression }\end{array}$ & $\begin{array}{l}\text { PET-PIB or } \\
\text { PET florbetapir } \\
\text { or PET } \\
\text { flutemetamol }\end{array}$ & $\begin{array}{c}\text { PET-Aß DEFINITION } 13 \\
\text { several isotopes. SUVR } \geq 1.5 \text { for } \\
11 \mathrm{C}-\mathrm{PiB}, \mathrm{SUVR} \geq 1.1 \text { for } 18 \mathrm{~F}- \\
\text { florbetapir, and } \mathrm{SUVR} \geq 0.62 \text { for } \\
18 \mathrm{~F} \text {-flutemetamol }\end{array}$ \\
\hline $\begin{array}{l}\text { (Lim, Snyder } \\
\text { et al. 2016) }\end{array}$ & 72 & Cognitive batttery & $\begin{array}{l}\text { Composite scores } \\
\text { (ADCS- } \\
\text { PACC/ZAVEN) }\end{array}$ & $\begin{array}{l}\text { PET-PIB or } \\
\text { PET florbetapir } \\
\text { or PET } \\
\text { flutemetamol }\end{array}$ & $\begin{array}{c}\text { PET-Aß DEFINITION } 13 \\
\text { PIB and flutemetamol SUVR } \geq \\
1.5 \\
\text { florbetapir SUVR } \geq 1.1\end{array}$ \\
\hline \multicolumn{6}{|c|}{ NEUROPATHOLOGICAL STUDIES } \\
\hline $\begin{array}{c}\text { (Jicha, } \\
\text { Abner et al. } \\
\text { 2012) }\end{array}$ & 12 & $\begin{array}{l}\text { NACC Cognitive } \\
\text { battery }\end{array}$ & $\begin{array}{l}\text { Cognitive } \\
\text { battery and } \\
\mathrm{NP}\end{array}$ & NP & $\begin{array}{c}\text { NP DEFINITION } 1 \\
\text { Braak staging and CERAD } \\
\text { plaques scores }\end{array}$ \\
\hline $\begin{array}{l}\text { (Abner, } \\
\text { Kryscio et al. } \\
2011)\end{array}$ & - & $\begin{array}{l}\text { MMSE/Clinical } \\
\text { dementia status }\end{array}$ & - & $\mathrm{NP}$ & $\begin{array}{c}\text { NP DEFINITION } 2 \\
\text { Braak stages V }\end{array}$ \\
\hline
\end{tabular}




\begin{tabular}{|c|c|c|c|c|c|}
\hline $\begin{array}{c}\text { Riley, Jicha } \\
\text { et al. 2011) }\end{array}$ & 900 & CERAD battery & NP & NP & $\begin{array}{c}\text { NP DEFINITION 3 } \\
\text { NIA-Reagan intermediate or } \\
\text { high-likelihood of AD }\end{array}$ \\
\hline $\begin{array}{c}\text { (Thal, von } \\
\text { Arnim et al. } \\
\text { 2013) }\end{array}$ & - & CDR & CDR & NP & $\begin{array}{c}\text { NP DEFINITION 1 } \\
\text { Thal phases for Aß and } \\
\text { Braak/CERAD stages for NFT }\end{array}$ \\
\hline
\end{tabular}

ADCS-PACC: alzheimer disease cooperative study-Preclinical Alzheimer Cognitive Composite CERAD: The Consortium to Establish a Registry for Alzheimer's Disease

NACC: National Alzheimer's Coordinating Center

NP: Neuropathology

PET: Positon Emission Tomograpy

PIB: Pittsburgh compound B

DVR: regional time-activity curves are analyzed for PIB specific binding by the Logan graphical analysis, using the cerebellum as a reference tissue input function, yielding a tracer distribution volume ratio or DVR.

MCBP: mean cortical binding potential. Regional DVR values are converted in an estimate of binding potential (DVR -1), and averaged to calculate the mean cortical value.

MMSE: Mini-mental State Examination

SUVR: PET standardized uptake value (SUV) data are summed and normalized to the cerebellar cortex SUV, yielding a region-to-cerebellar ratio named SUVR

Abner, E. L., R. J. Kryscio, F. A. Schmitt, K. S. Santacruz, G. A. Jicha, Y. Lin, J. M. Neltner, C. D. Smith, L. J. Van Eldik and P. T. Nelson (2011). ""End-stage" neurofibrillary tangle pathology in preclinical Alzheimer's disease: fact or fiction?" J Alzheimers Dis 25(3): 445453.

Alcolea, D., P. Martinez-Lage, P. Sanchez-Juan, J. Olazaran, C. Antunez, A. Izagirre, M. Ecay-Torres, A. Estanga, M. Clerigue, M. C. Guisasola, D. Sanchez Ruiz, J. Marin Munoz, M. Calero, R. Blesa, J. Clarimon, M. Carmona-Iragui, E. Morenas-Rodriguez, E. RodriguezRodriguez, J. L. Vazquez Higuera, J. Fortea and A. Lleo (2015). "Amyloid precursor protein metabolism and inflammation markers in preclinical Alzheimer disease." Neurology 85(7): 626-633.

Amariglio, R. E., J. A. Becker, J. Carmasin, L. P. Wadsworth, N. Lorius, C. Sullivan, J. E. Maye, C. Gidicsin, L. C. Pepin, R. A. Sperling, K. A. Johnson and D. M. Rentz (2012).

"Subjective cognitive complaints and amyloid burden in cognitively normal older individuals." Neuropsychologia 50(12): 2880-2886.

Ayutyanont, N., J. B. Langbaum, S. B. Hendrix, K. Chen, A. S. Fleisher, M. Friesenhahn, M. Ward, C. Aguirre, N. Acosta-Baena, L. Madrigal, C. Munoz, V. Tirado, S. Moreno, P. N. Tariot, F. Lopera and E. M. Reiman (2014). "The Alzheimer's prevention initiative composite cognitive test score: sample size estimates for the evaluation of preclinical Alzheimer's disease treatments in presenilin 1 E280A mutation carriers." J Clin Psychiatry 75(6): 652-660. Bilgel, M., J. L. Prince, D. F. Wong, S. M. Resnick and B. M. Jedynak (2016). "A multivariate nonlinear mixed effects model for longitudinal image analysis: Application to amyloid imaging." Neuroimage 134: 658-670.

Brier, M. R., J. E. McCarthy, T. L. Benzinger, A. Stern, Y. Su, K. A. Friedrichsen, J. C. Morris, B. M. Ances and A. G. Vlassenko (2016). "Local and distributed PiB accumulation associated with development of preclinical Alzheimer's disease." Neurobiol Aging 38: 104111.

Brier, M. R., J. B. Thomas, A. M. Fagan, J. Hassenstab, D. M. Holtzman, T. L. Benzinger, J. C. Morris and B. M. Ances (2014). "Functional connectivity and graph theory in preclinical Alzheimer's disease." Neurobiol Aging 35(4): 757-768. 
Brier, M. R., J. B. Thomas, A. Z. Snyder, L. Wang, A. M. Fagan, T. Benzinger, J. C. Morris and B. M. Ances (2014). "Unrecognized preclinical Alzheimer disease confounds rs-fcMRI studies of normal aging." Neurology 83(18): 1613-1619.

Clark, L. R., A. M. Racine, R. L. Koscik, O. C. Okonkwo, C. D. Engelman, C. M. Carlsson, S. Asthana, B. B. Bendlin, R. Chappell, C. R. Nicholas, H. A. Rowley, J. M. Oh, B. P. Hermann, M. A. Sager, B. T. Christian and S. C. Johnson (2016). "Beta-amyloid and cognitive decline in late middle age: Findings from the Wisconsin Registry for Alzheimer's Prevention study." Alzheimers Dement.

Craig-Schapiro, R., R. J. Perrin, C. M. Roe, C. Xiong, D. Carter, N. J. Cairns, M. A. Mintun, E. R. Peskind, G. Li, D. R. Galasko, C. M. Clark, J. F. Quinn, G. D'Angelo, J. P. Malone, R. R. Townsend, J. C. Morris, A. M. Fagan and D. M. Holtzman (2010). "YKL-40: a novel prognostic fluid biomarker for preclinical Alzheimer's disease." Biol Psychiatry 68(10): 903912.

Desikan, R. S., L. K. McEvoy, W. K. Thompson, D. Holland, J. B. Brewer, P. S. Aisen, R. A. Sperling, A. M. Dale and I. Alzheimer's Disease Neuroimaging (2012). "Amyloid-beta-associated clinical decline occurs only in the presence of elevated P-tau." Arch Neurol 69(6): 709-713.

Doherty, B. M., S. A. Schultz, J. M. Oh, R. L. Koscik, N. M. Dowling, T. E. Barnhart, D. Murali, C. L. Gallagher, C. M. Carlsson, B. B. Bendlin, A. LaRue, B. P. Hermann, H. A. Rowley, S. Asthana, M. A. Sager, B. T. Christian, S. C. Johnson and O. C. Okonkwo (2015). "Amyloid burden, cortical thickness, and cognitive function in the Wisconsin Registry for Alzheimer's Prevention." Alzheimers Dement (Amst) 1(2): 160-169.

Donohue, M. C., R. A. Sperling, D. P. Salmon, D. M. Rentz, R. Raman, R. G. Thomas, M. Weiner, P. S. Aisen, B. Australian Imaging, A. Lifestyle Flagship Study of, I. Alzheimer's Disease Neuroimaging and S. Alzheimer's Disease Cooperative (2014). "The preclinical Alzheimer cognitive composite: measuring amyloid-related decline." JAMA Neurol 71(8): 961-970.

Fortea, J., E. Vilaplana, D. Alcolea, M. Carmona-Iragui, M. B. Sanchez-Saudinos, I. Sala, S. Anton-Aguirre, S. Gonzalez, S. Medrano, J. Pegueroles, E. Morenas, J. Clarimon, R. Blesa, A. Lleo and I. Alzheimer's Disease Neuroimaging (2014). "Cerebrospinal fluid beta-amyloid and phospho-tau biomarker interactions affecting brain structure in preclinical Alzheimer disease." Ann Neurol 76(2): 223-230.

Harrington, K. D., E. Gould, Y. Y. Lim, D. Ames, R. H. Pietrzak, A. Rembach, S. RaineySmith, R. N. Martins, O. Salvado, V. L. Villemagne, C. C. Rowe, C. L. Masters, P. Maruff and A. R. Group (2016). "Amyloid burden and incident depressive symptoms in cognitively normal older adults." Int J Geriatr Psychiatry.

Harrington, M. G., J. Chiang, J. M. Pogoda, M. Gomez, K. Thomas, S. D. Marion, K. J. Miller, P. Siddarth, X. Yi, F. Zhou, S. Lee, X. Arakaki, R. P. Cowan, T. Tran, C. Charleswell, B. D. Ross and A. N. Fonteh (2013). "Executive function changes before memory in preclinical Alzheimer's pathology: a prospective, cross-sectional, case control study." PLoS One 8(11): e79378.

Hassenstab, J., D. Ruvolo, M. Jasielec, C. Xiong, E. Grant and J. C. Morris (2015). "Absence of practice effects in preclinical Alzheimer's disease." Neuropsychology 29(6): 940-948. Jack, C. R., Jr., D. S. Knopman, S. D. Weigand, H. J. Wiste, P. Vemuri, V. Lowe, K. Kantarci, J. L. Gunter, M. L. Senjem, R. J. Ivnik, R. O. Roberts, W. A. Rocca, B. F. Boeve and R. C. Petersen (2012). "An operational approach to National Institute on Aging-Alzheimer's Association criteria for preclinical Alzheimer disease." Ann Neurol 71(6): 765-775. Jack, C. R., Jr., H. J. Wiste, S. D. Weigand, D. S. Knopman, M. M. Mielke, P. Vemuri, V. Lowe, M. L. Senjem, J. L. Gunter, D. Reyes, M. M. Machulda, R. Roberts and R. C. Petersen 
(2015). "Different definitions of neurodegeneration produce similar amyloid/neurodegeneration biomarker group findings." Brain 138(Pt 12): 3747-3759. Jack, C. R., Jr., H. J. Wiste, S. D. Weigand, W. A. Rocca, D. S. Knopman, M. M. Mielke, V. J. Lowe, M. L. Senjem, J. L. Gunter, G. M. Preboske, V. S. Pankratz, P. Vemuri and R. C. Petersen (2014). "Age-specific population frequencies of cerebral beta-amyloidosis and neurodegeneration among people with normal cognitive function aged 50-89 years: a crosssectional study." Lancet Neurol 13(10): 997-1005.

Jicha, G. A., E. L. Abner, F. A. Schmitt, R. J. Kryscio, K. P. Riley, G. E. Cooper, N. Stiles, M. S. Mendiondo, C. D. Smith, L. J. Van Eldik and P. T. Nelson (2012). "Preclinical AD Workgroup staging: pathological correlates and potential challenges." Neurobiol Aging 33(3): 622 e621-622 e616.

Ju, Y. E., J. S. McLeland, C. D. Toedebusch, C. Xiong, A. M. Fagan, S. P. Duntley, J. C. Morris and D. M. Holtzman (2013). "Sleep quality and preclinical Alzheimer disease." JAMA Neurol 70(5): 587-593.

Knopman, D. S., C. R. Jack, Jr., H. J. Wiste, S. D. Weigand, P. Vemuri, V. Lowe, K. Kantarci, J. L. Gunter, M. L. Senjem, R. J. Ivnik, R. O. Roberts, B. F. Boeve and R. C. Petersen (2012).

"Short-term clinical outcomes for stages of NIA-AA preclinical Alzheimer disease."

Neurology 78(20): 1576-1582.

Knopman, D. S., C. R. Jack, Jr., H. J. Wiste, S. D. Weigand, P. Vemuri, V. J. Lowe, K. Kantarci, J. L. Gunter, M. L. Senjem, M. M. Mielke, R. O. Roberts, B. F. Boeve and R. C. Petersen (2013). "Brain injury biomarkers are not dependent on beta-amyloid in normal elderly." Ann Neurol 73(4): 472-480.

Lim, Y. Y., P. Maruff, R. H. Pietrzak, D. Ames, K. A. Ellis, K. Harrington, N. T. Lautenschlager, C. Szoeke, R. N. Martins, C. L. Masters, V. L. Villemagne, C. C. Rowe and A. R. Group (2014). "Effect of amyloid on memory and non-memory decline from preclinical to clinical Alzheimer's disease." Brain 137(Pt 1): 221-231.

Lim, Y. Y., P. J. Snyder, R. H. Pietrzak, A. Ukiqi, V. L. Villemagne, D. Ames, O. Salvado, P. Bourgeat, R. N. Martins, C. L. Masters, C. C. Rowe and P. Maruff (2016). "Sensitivity of composite scores to amyloid burden in preclinical Alzheimer's disease: Introducing the Zscores of Attention, Verbal fluency, and Episodic memory for Nondemented older adults composite score." Alzheimers Dement (Amst) 2: 19-26.

Lim, Y. Y., V. L. Villemagne, S. M. Laws, D. Ames, R. H. Pietrzak, K. A. Ellis, K. D. Harrington, P. Bourgeat, O. Salvado, D. Darby, P. J. Snyder, A. I. Bush, R. N. Martins, C. L. Masters, C. C. Rowe, P. J. Nathan, P. Maruff, B. Australian Imaging and G. Lifestyle Research (2013). "BDNF Val66Met, Abeta amyloid, and cognitive decline in preclinical Alzheimer's disease." Neurobiol Aging 34(11): 2457-2464.

Mielke, M. M., H. J. Wiste, S. D. Weigand, D. S. Knopman, V. J. Lowe, R. O. Roberts, Y. E. Geda, D. M. Swenson-Dravis, B. F. Boeve, M. L. Senjem, P. Vemuri, R. C. Petersen and C. R. Jack, Jr. (2012). "Indicators of amyloid burden in a population-based study of cognitively normal elderly." Neurology 79(15): 1570-1577.

Mormino, E. C., R. A. Betensky, T. Hedden, A. P. Schultz, R. E. Amariglio, D. M. Rentz, K. A. Johnson and R. A. Sperling (2014). "Synergistic effect of beta-amyloid and neurodegeneration on cognitive decline in clinically normal individuals." JAMA Neurol 71(11): 1379-1385.

Mormino, E. C., R. A. Betensky, T. Hedden, A. P. Schultz, A. Ward, W. Huijbers, D. M. Rentz, K. A. Johnson, R. A. Sperling, I. Alzheimer's Disease Neuroimaging, B. Australian Imaging, A. Lifestyle Flagship Study of and S. Harvard Aging Brain (2014). "Amyloid and APOE epsilon4 interact to influence short-term decline in preclinical Alzheimer disease." Neurology 82(20): 1760-1767. 
Morris, J. C., C. M. Roe, E. A. Grant, D. Head, M. Storandt, A. M. Goate, A. M. Fagan, D. M. Holtzman and M. A. Mintun (2009). "Pittsburgh Compound B imaging and prediction of progression from cognitive normality to symptomatic Alzheimer disease." Archives of Neurology 66(12): 1469-1475.Morris, J. C., C. M. Roe, C. Xiong, A. M. Fagan, A. M. Goate, D. M. Holtzman and M. A. Mintun (2010). "APOE predicts amyloid-beta but not tau Alzheimer pathology in cognitively normal aging." Ann Neurol 67(1): 122-131.

Nation, D. A., E. C. Edmonds, K. J. Bangen, L. Delano-Wood, B. K. Scanlon, S. D. Han, S. D. Edland, D. P. Salmon, D. R. Galasko, M. W. Bondi and I. Alzheimer's Disease Neuroimaging Initiative (2015). "Pulse pressure in relation to tau-mediated neurodegeneration, cerebral amyloidosis, and progression to dementia in very old adults." JAMA Neurol 72(5): 546-553. Papp, K. V., R. E. Amariglio, E. C. Mormino, T. Hedden, M. Dekhytar, K. A. Johnson, R. A. Sperling and D. M. Rentz (2015). "Free and cued memory in relation to biomarker-defined abnormalities in clinically normal older adults and those at risk for Alzheimer's disease." Neuropsychologia 73: 169-175.

Papp, K. V., E. C. Mormino, R. E. Amariglio, C. Munro, A. Dagley, A. P. Schultz, K. A. Johnson, R. A. Sperling and D. M. Rentz (2016). "Biomarker validation of a decline in semantic processing in preclinical Alzheimer's disease." Neuropsychology 30(5): 624-630. Pascoal, T. A., S. Mathotaarachchi, S. Mohades, A. L. Benedet, C. O. Chung, M. Shin, S. Wang, T. Beaudry, M. S. Kang, J. P. Soucy, A. Labbe, S. Gauthier and P. Rosa-Neto (2016). "Amyloid-beta and hyperphosphorylated tau synergy drives metabolic decline in preclinical Alzheimer's disease." Mol Psychiatry.

Pettigrew, C., A. Soldan, A. Moghekar, M. C. Wang, A. L. Gross, R. O'Brien and M. Albert (2015). "Relationship between cerebrospinal fluid biomarkers of Alzheimer's disease and cognition in cognitively normal older adults." Neuropsychologia 78: 63-72.

Pietrzak, R. H., Y. Y. Lim, D. Ames, K. Harrington, C. Restrepo, R. N. Martins, A. Rembach, S. M. Laws, C. L. Masters, V. L. Villemagne, C. C. Rowe, P. Maruff, B. Australian Imaging and G. Lifestyle Research (2015). "Trajectories of memory decline in preclinical Alzheimer's disease: results from the Australian Imaging, Biomarkers and Lifestyle Flagship Study of ageing." Neurobiol Aging 36(3): 1231-1238.

Pietrzak, R. H., Y. Y. Lim, A. Neumeister, D. Ames, K. A. Ellis, K. Harrington, N. T. Lautenschlager, C. Restrepo, R. N. Martins, C. L. Masters, V. L. Villemagne, C. C. Rowe, P. Maruff, B. Australian Imaging and G. Lifestyle Research (2015). "Amyloid-beta, anxiety, and cognitive decline in preclinical Alzheimer disease: a multicenter, prospective cohort study." JAMA Psychiatry 72(3): 284-291.

Pike, K. E., K. A. Ellis, V. L. Villemagne, N. Good, G. Chetelat, D. Ames, C. Szoeke, S. M. Laws, G. Verdile, R. N. Martins, C. L. Masters and C. C. Rowe (2011). "Cognition and betaamyloid in preclinical Alzheimer's disease: data from the AIBL study." Neuropsychologia 49(9): 2384-2390.

Racine, A. M., N. Adluru, A. L. Alexander, B. T. Christian, O. C. Okonkwo, J. Oh, C. A. Cleary, A. Birdsill, A. T. Hillmer, D. Murali, T. E. Barnhart, C. L. Gallagher, C. M. Carlsson, H. A. Rowley, N. M. Dowling, S. Asthana, M. A. Sager, B. B. Bendlin and S. C. Johnson (2014). "Associations between white matter microstructure and amyloid burden in preclinical Alzheimer's disease: A multimodal imaging investigation." Neuroimage Clin 4: 604-614. Racine, A. M., R. L. Koscik, S. E. Berman, C. R. Nicholas, L. R. Clark, O. C. Okonkwo, H. A. Rowley, S. Asthana, B. B. Bendlin, K. Blennow, H. Zetterberg, C. E. Gleason, C. M. Carlsson and S. C. Johnson (2016). "Biomarker clusters are differentially associated with longitudinal cognitive decline in late midlife." Brain.

Riley, K. P., G. A. Jicha, D. Davis, E. L. Abner, G. E. Cooper, N. Stiles, C. D. Smith, R. J. Kryscio, P. T. Nelson, L. J. Van Eldik and F. A. Schmitt (2011). "Prediction of preclinical 
Alzheimer's disease: longitudinal rates of change in cognition." J Alzheimers Dis 25(4): 707717.

Soldan, A., C. Pettigrew, Q. Cai, M. C. Wang, A. R. Moghekar, R. J. O'Brien, O. A. Selnes, M. S. Albert and B. R. Team (2016). "Hypothetical Preclinical Alzheimer Disease Groups and Longitudinal Cognitive Change." JAMA Neurol 73(6): 698-705.

Stark, S. L., C. M. Roe, E. A. Grant, H. Hollingsworth, T. L. Benzinger, A. M. Fagan, V. D. Buckles and J. C. Morris (2013). "Preclinical Alzheimer disease and risk of falls." Neurology 81(5): 437-443.

Sutphen, C. L., M. S. Jasielec, A. R. Shah, E. M. Macy, C. Xiong, A. G. Vlassenko, T. L. Benzinger, E. E. Stoops, H. M. Vanderstichele, B. Brix, H. D. Darby, M. L. Vandijck, J. H. Ladenson, J. C. Morris, D. M. Holtzman and A. M. Fagan (2015). "Longitudinal Cerebrospinal Fluid Biomarker Changes in Preclinical Alzheimer Disease During Middle Age." JAMA Neurol 72(9): 1029-1042.

Thai, C., Y. Y. Lim, V. L. Villemagne, S. M. Laws, D. Ames, K. A. Ellis, S. R. Rainey-Smith, R. N. Martins, C. L. Masters, C. C. Rowe, P. Maruff, B. Australian Imaging and G. Lifestyle Research (2015). "Amyloid-Related Memory Decline in Preclinical Alzheimer's Disease Is Dependent on APOE epsilon4 and Is Detectable over 18-Months." PLoS One 10(10): e0139082.

Thal, D. R., C. von Arnim, W. S. Griffin, H. Yamaguchi, R. E. Mrak, J. Attems and A. R. Upadhaya (2013). "Pathology of clinical and preclinical Alzheimer's disease." Eur Arch Psychiatry Clin Neurosci 263 Suppl 2: S137-145.

Valech, N., M. A. Mollica, J. Olives, A. Tort, J. Fortea, A. Lleo, S. S. Belen, J. L. Molinuevo and L. Rami (2015). "Informants' Perception of Subjective Cognitive Decline Helps to Discriminate Preclinical Alzheimer's Disease from Normal Aging." J Alzheimers Dis 48 Suppl 1: S87-98.

van Harten, A. C., L. L. Smits, C. E. Teunissen, P. J. Visser, T. Koene, M. A. Blankenstein, P. Scheltens and W. M. van der Flier (2013). "Preclinical AD predicts decline in memory and executive functions in subjective complaints." Neurology 81(16): 1409-1416.

Voevodskaya, O., P. C. Sundgren, O. Strandberg, H. Zetterberg, L. Minthon, K. Blennow, L. O. Wahlund, E. Westman, O. Hansson and F. s. g. Swedish Bio (2016). "Myo-inositol changes precede amyloid pathology and relate to APOE genotype in Alzheimer disease." Neurology 86(19): 1754-1761.

Vos, S. J., B. A. Gordon, Y. Su, P. J. Visser, D. M. Holtzman, J. C. Morris, A. M. Fagan and T. L. Benzinger (2016). "NIA-AA staging of preclinical Alzheimer disease: discordance and concordance of CSF and imaging biomarkers." Neurobiol Aging 44: 1-8.

Vos, S. J., C. Xiong, P. J. Visser, M. S. Jasielec, J. Hassenstab, E. A. Grant, N. J. Cairns, J. C. Morris, D. M. Holtzman and A. M. Fagan (2013). "Preclinical Alzheimer's disease and its outcome: a longitudinal cohort study." Lancet Neurol 12(10): 957-965.

Wang, L., T. L. Benzinger, J. Hassenstab, T. Blazey, C. Owen, J. Liu, A. M. Fagan, J. C. Morris and B. M. Ances (2015). "Spatially distinct atrophy is linked to beta-amyloid and tau in preclinical Alzheimer disease." Neurology 84(12): 1254-1260.

Whitwell, J. L., N. Tosakulwong, S. D. Weigand, M. L. Senjem, V. J. Lowe, J. L. Gunter, B. F. Boeve, D. S. Knopman, B. C. Dickerson, R. C. Petersen and C. R. Jack, Jr. (2013). "Does amyloid deposition produce a specific atrophic signature in cognitively normal subjects?" Neuroimage Clin 2: 249-257. 
Supplementary Table 3

\begin{tabular}{|c|c|}
\hline Cohort & Npsy Battery \\
\hline ADNI1-GO-2 & $\begin{array}{c}\text { ADAS-Cog, American National Adult Reading Test*, AVLT, BNT, } \\
\text { Category Fluency, CDR Clock Drawing, Digit Span, DSST, e-Cog* } \\
\text { Logical Memory Test II, } \\
\text { MMSE, MoCA, Number Cancellation, TMT } \\
{ }^{*} \text { ADNI-GO and } 2 \text { only) }\end{array}$ \\
\hline AMSTERDAM Dementia Cohort & $\begin{array}{c}\text { AVLT, Cambridge Cognitive Examination for Global Cognitive } \\
\text { Decline, Digit Span, MMSE, TMT, Verbal Fluency (categories), } \\
\text { Verbal Fluency (letters), Visual Association Test, Visual Object } \\
\text { and Space Perception Battery }\end{array}$ \\
\hline $\begin{array}{l}\text { AIBL study (Australian Imaging, Biomarkers and Lifestyle } \\
\text { study) }\end{array}$ & Cogstate Brief Battery, CDR, CVLT, MMSE \\
\hline $\begin{array}{c}\text { BIOCARD } \\
\text { (Prospective Study of Biomarkers for Older Controls at Risk for } \\
\text { Alzheimer's Disease) }\end{array}$ & $\begin{array}{c}\text { BNT, Buschke FCSRT, Verbal Fluency (animals), CDR, CVLT, } \\
\text { Digit Span, DSST, Lafayette Grooved Pegboard test Letter } \\
\text { Fluency, MMSE, Rey-Osterreith Complex Figure, TMT, WAIS-R, } \\
\text { WMS-R }\end{array}$ \\
\hline $\begin{array}{l}\text { BioFINDER (Biomarkers For Identifying Neurodegenerative } \\
\text { Disorders Early and Reliably cognitively healthy cohort) }\end{array}$ & $\begin{array}{l}\text { A Quick Test of Cognitive speed (AQT), AVLT, Block Design, } \\
\text { Verbal Fluency (animals), Clock Drawing, DSST, Verbal Fluency } \\
\text { (letters), MMSE, Months Backwards, Multiple Choice Vocabulary } \\
\text { Test (SRB:1), Rey-Ostereith Complex Figure, Stroop, TMT }\end{array}$ \\
\hline $\begin{array}{c}\text { BLSA } \\
\text { (Baltimore longitudinal study of Aging) }\end{array}$ & $\begin{array}{c}\text { Benton visual Retention Test, Blessed Information- Memory- } \\
\text { Concentration Test, BNT, CVLT, Card Retention Test, Verbal } \\
\text { Fluency (animals), Verbal Fluency (letters), Digit Span, MMSE, } \\
\text { TMT }\end{array}$ \\
\hline $\begin{array}{c}\text { HABS } \\
\text { (Harvard Aging Brain Study) }\end{array}$ & $\begin{array}{c}\text { Benton Visual Discrimimation Test, BNT, CDR, e-Cog, Letter- } \\
\text { Number Sequencing, Memory Functioning Questionnaire, MMSE, } \\
\text { 6-trial Selective Reminding Test, TMT }\end{array}$ \\
\hline $\begin{array}{c}\text { MCSA } \\
\text { (Mayo Clinic Study of Aging) }\end{array}$ & $\begin{array}{l}\text { AVLT, BNT, CDR, TMT, Verbal Fluency (animals), WAIS-R, WMS- } \\
\text { R }\end{array}$ \\
\hline $\begin{array}{c}\text { NACC } \\
\text { (National Alzheimer's Coordinating Center database) }\end{array}$ & $\begin{array}{l}\text { BNT, Digit Span, Logical Memory (WMS-R), MMSE, DSST, TMT, } \\
\text { Verbal Fluency (animals), }\end{array}$ \\
\hline Nun Study & $\begin{array}{c}\text { CERAD Battery (i.e.BNT, Constructional Praxis Word List Memory } \\
\text { Delayed Recall, Recognition; Verbal Fluency), MMSE }\end{array}$ \\
\hline SIGNAL & $\begin{array}{c}\text { BNT, Buschke FCSRT, Verbal Fluency (animals), Verbal Fluency } \\
\text { (letters, Logical Memory (WMS-R), Rey-Osterrieth Complex } \\
\text { Figure Test, TMT, Word list (CERAD) }\end{array}$ \\
\hline $\begin{array}{l}\text { WU-ADRC (Washington University's Alzheimer's Disease } \\
\text { Research Center study) }\end{array}$ & $\begin{array}{c}\text { Paired associate learning (WMS-R), Benton Visual Retention } \\
\text { Test, Block Design, Boston Naming Test, CDR, Digit Span, DSST, } \\
\text { Information, Logical Memory test (WAIS-R), Verbal fluency } \\
\text { (letter), Mental Control, TMT }\end{array}$ \\
\hline $\begin{array}{c}\text { WRAP } \\
\text { (Wisconsin Registry for Alzheimer's Prevention) }\end{array}$ & $\begin{array}{l}\text { AVLT, BNT, COWA, Face Recognition (WMS), Judgement of Line } \\
\text { Orientation, Stroop, TMT, WASI, Wisconsin Card-Sort-64, WRAT- } \\
3\end{array}$ \\
\hline
\end{tabular}

Abbreviations:

\begin{tabular}{|l|l|}
\hline ADAS-Cog & Alzheimer Disease Assessment Scale-Cognition \\
\hline AVLT & Rey Auditory Verbal Learning Task \\
\hline BNT & Boston Naming Test \\
\hline Benton & Benton Visual Retention Test \\
\hline COWA & Controlled Oral Word Association \\
\hline CVLT & California Verbal Learning Test \\
\hline DSST & Digit Symbol Substitution Test \\
\hline FCSRT & Free and Cued Selective Reminding Test \\
\hline MMSE & Mini-mental State Evaluation \\
\hline MoCA & Montreal Cognitive Assessment \\
\hline TMT & Trail Making Test (Reitan) \\
\hline WAIS & Wechsler Adult Intelligence Scale \\
\hline WART & Wide Range Achievement Reading Test \\
\hline WASI & Wechsler Abbreviated Scale of Intelligence \\
\hline
\end{tabular}


